Supplemental Information for

\title{
Reversible Photohydration of Trenbolone Acetate Metabolites: Mechanistic Understanding of Product-to-Parent Reversion through Complementary Experimental and Theoretical Approaches
}

\author{
Jonas Baltrusaitis, ${ }^{1,2^{*}}$ Eric V. Patterson, ${ }^{3}$ Meghan O'Connor, ${ }^{4}$ Shen $\mathrm{Qu},{ }^{4}$ \\ Edward P. Kolodziej, ${ }^{5,6}$ and David M. Cwiertny ${ }^{4,7^{*}}$
}

${ }^{1}$ Department of Chemical and Biomolecular Engineering, Lehigh University, B336 Iacocca Hall, 111 Research Drive, Bethlehem, PA 18015

${ }^{2}$ College of Public Health, University of Iowa, Iowa City, IA 52242

${ }^{3}$ Department of Chemistry, Stony Brook University, Stony Brook, NY 11794

${ }^{4}$ Department of Civil and Environmental Engineering, University of Iowa, 4105 Seamans Center for the Engineering Arts and Sciences, Iowa City, IA, 52242

${ }^{5}$ Interdisciplinary Arts and Sciences, University of Washington, Tacoma, Tacoma WA 98402

${ }^{6}$ Civil and Environmental Engineering, University of Washington, Seattle, WA 98195

${ }^{7}$ Department of Chemical and Biochemical Engineering, University of Iowa, Iowa City, IA 52242

Corresponding Authors' Contact Information:

Jonas Baltrusaitis: job314@lehigh.edu (email); 610-758-6836 (phone)

David M. Cwiertny: david-cwiertny@uiowa.edu (e-mail); 319-335-1401 (phone)

\section{Environmental Science and Technology}

Prepared January 13, 2015

77 Pages, 2 Tables, 6 Figures, 3 Schemes

Supplemental Information contains the list of reagents, details about the light source, discussion of results from $\mathrm{D}_{2} \mathrm{O}$ experiments, tabulated values of thermal decay coefficients for photohydrates, and figures related to the $\mathrm{pH}$-dependent photolysis of $17 \alpha-\mathrm{TBOH}$, photolysis results in $\mathrm{D}_{2} \mathrm{O}$, assessment of trenbolone analog formation via carbocation rearrangement, and additional computational details, including Cartesian coordinates. 


\section{Table of Contents}

I. Supplemental Experimental Methods ..................................................................................... 2

II. Supplemental Results and Discussion ................................................................................ 3

III. Supplemental Tables and Figures (Table S1 and Figures S1-S6) .................................... 4

IV. Additional Computational Details...................................................................................... 11

Mechanistic Schemes.................................................................................................................................... 11

Cartesian Coordinates of Optimized Structures ..................................................................................... 14

Structures for neutral, photochemical hydration ....................................................................... 14

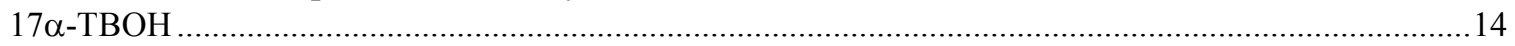

$17 \beta-\mathrm{TBOH}$

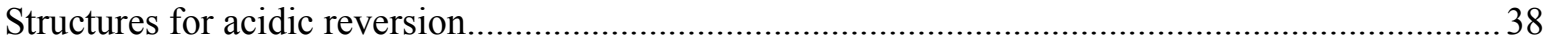

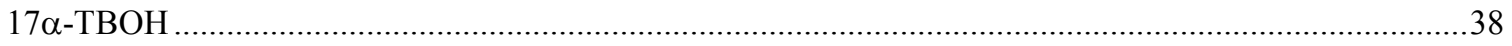

$17 \beta-\mathrm{TBOH}$

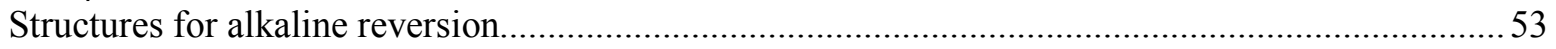

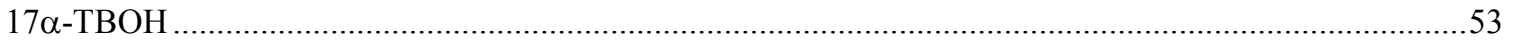

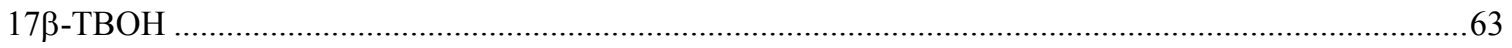

Details of CAS (10,10) Active Space (Figure S7) ................................................................................74

Additional Details for Structures Involved in Photohydration Pathway (Table S2)....................... 75

VI. Literature Cited................................................................................................................... 77 


\section{Supplemental Experimental Methods}

Reagents. $17 \beta-\mathrm{TBOH}$ (Steraloids; >99\%) and $17 \alpha-\mathrm{TBOH}$ (Cerilliant; 99\%) were acquired from commercial sources and used as received. Direct photolysis experiments were conducted in either a $5 \mathrm{mM}$ phosphate buffer (at $\mathrm{pH} 5$ and 7) prepared from potassium phosphate (Fisher; 99\%) or a $25 \mathrm{mM}$ borate (pH 9) buffer prepared from boric acid (Sigma-Aldrich; 99.9\%). When necessary, a small volume of $1 \mathrm{M} \mathrm{HCl}$ [prepared from concentrated $\mathrm{HCl}$ (Fisher; $\mathrm{NF} / \mathrm{FCC}$ grade)] or $1 \mathrm{M} \mathrm{NaOH}$ (ACS Reagent; $\geq 97 \%$ ) solutions were used to adjust the $\mathrm{pH}$ of these buffer solutions to the desired initial value. Stock solutions of each TBA metabolite were prepared by dissolving a known mass of each compound in methanol (Fisher; 99.9\%) to achieve a final concentration of $10 \mathrm{mM}$. All aqueous solutions were prepared in deionized water (Millipore; Milli-Q). Solvent kinetic isotope effect experiments used deuterium oxide $\left(\mathrm{D}_{2} \mathrm{O}\right.$, Aldrich, 99.9 atom \% D).

Additional details of photoreactor and light source. Experiments were conducted in a water-jacketed, borosilicate photoreactor $(37 \mathrm{~mm}$ inner diameter $\times 67 \mathrm{~mm}$ depth for a nominal volume of $\sim 50 \mathrm{~mL}$; Chemglass), whose contents were mixed via a magnetic stirrer and stir plate during all experiments. Experiments used $25 \mathrm{~mL}$ of TBA metabolite solution in an appropriate buffer, and the system temperature was held constant via a recirculating water bath. Light from the arc lamp was first passed through a water filter to remove IR radiation, reflected off a $90^{\circ}$ full reflectance beam turning mirror, and then passed through a $305 \mathrm{~nm}$ long-pass filter to generate wavelengths of light more closely resembling those available at earth's surface. Lamp irradiance for wavelengths greater than $250 \mathrm{~nm}$ was measured with a spectroradiometer (ILT950; International Light Technologies, Peabody, MA) at reactor height. Calculations described elsewhere (Nalbandian et al., 2015) were used to determine a photon fluence of $\sim 6 \times 10^{15}$ photons $/ \mathrm{cm}^{2} \cdot \mathrm{s}$ between 305 and $400 \mathrm{~nm}$, which we assumed was the primary range of absorbable photons for TBA metabolites $\left(\lambda_{\max } \sim 350 \mathrm{~nm}\right)$. 


\section{Supplemental Results and Discussion}

Solvent isotope effects for the reversible photohydration of $17 \alpha-\mathrm{TBOH}$ and $17 \beta-$

TBOH in $\mathbf{D}_{2} \mathrm{O}$. For both $17 \alpha-$ and $17 \beta-\mathrm{TBOH}$, photohydrate yields in $\mathrm{D}_{2} \mathrm{O}$ as a function of reaction progress (Figure S3) generally match those in $\mathrm{H}_{2} \mathrm{O}$, with one notable exception. At acidic $\mathrm{pD}$, interconversion of 5-OH-TBOH to $12-\mathrm{OH}-\mathrm{TBOH}$ is inhibited, consistent with greater stability of 5-OH-TBOH in $\mathrm{D}_{2} \mathrm{O}$. Indeed, from photohydrate thermal (dark) stability data (Figure S4), 5-OH-TBOH is considerably more stable in $\mathrm{D}_{2} \mathrm{O}$ than in $\mathrm{H}_{2} \mathrm{O}$ at both acidic and basic pD values. At pD 5, it is clear that 5-OH-TBOH to $12-\mathrm{OH}-\mathrm{TBOH}$ interconversion can still occur (albeit at a slower rate than in $\mathrm{H}_{2} \mathrm{O}$ ) because of an obvious increase (by $\sim 40 \%$ ) in $12-\mathrm{OH}-17 \alpha-$ $\mathrm{TBOH}$ concentration over roughly $\sim 24 \mathrm{~h}$ in the dark. At neutral $\mathrm{pD}$, the rate of $5-\mathrm{OH}-\mathrm{TBOH}$ decay is slightly greater in $\mathrm{D}_{2} \mathrm{O}$ than in $\mathrm{H}_{2} \mathrm{O}$, whereas far greater stability of $12-\mathrm{OH}-\mathrm{TBOH}$ is observed at neutral pD, with small increases in its concentration over time even suggesting some degree of 5-OH-TBOH to $12-\mathrm{OH}-\mathrm{TBOH}$ interconversion at pD 7. Generally, the greater stability of 5-OH-TBOH observed in $\mathrm{D}_{2} \mathrm{O}$ results in slower initial rates of TBA metabolite regeneration via dehydration across all $\mathrm{pD}$ values (Figure S5). In only one system, $17 \alpha-\mathrm{TBOH}$ at $\mathrm{pD} 5$, was a greater extent of reversion observed in $\mathrm{D}_{2} \mathrm{O}$, which presumably is attributable to the greater accumulation 5-OH-TBOH because of its stability in $\mathrm{D}_{2} \mathrm{O}$. 


\section{Supplemental Tables and Figures}

Table S1. First order rate constants $\left(k\right.$ values in $\left.\mathrm{h}^{-1}\right)$ for photohydrate thermal decay. Uncertainties represent $95 \%$ confidence intervals associated with linear regression analysis of semi-log concentration versus time data (aggregated from at least duplicate experiments) used to determine $k$ values.

\begin{tabular}{cccc} 
& & 5OH-TBOH & 120H-TBOH \\
\hline pH 5 & $17 \alpha-\mathrm{TBOH}$ & $7.1( \pm 0.6) \times 10^{-1}$ & $1.68( \pm 0.04) \times 10^{-2}$ \\
& $17 \beta-\mathrm{TBOH}$ & $5.9( \pm 1.2) \times 10^{-1}$ & $1.63( \pm 0.02) \times 10^{-2}$ \\
\hline pH 7 & $17 \alpha-\mathrm{TBOH}$ & $1.42( \pm 0.02) \times 10^{-2}$ & $1.73( \pm 0.01) \times 10^{-2}$ \\
& $17 \beta-\mathrm{TBOH}$ & $2.90( \pm 0.10) \times 10^{-2}$ & $3.8( \pm 0.2) \times 10^{-2}$ \\
\hline pH 9 & $17 \alpha-\mathrm{TBOH}$ & $5.18( \pm 0.09) \times 10^{-2}$ & $5.7( \pm 0.5) \times 10^{-2}$ \\
& $17 \beta-\mathrm{TBOH}$ & $4.8( \pm 0.7) \times 10^{-2}$ & $3.9( \pm 0.9) \times 10^{-1}$ \\
\hline \hline
\end{tabular}




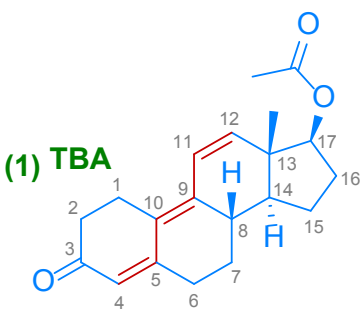

Trenbolone Acetate

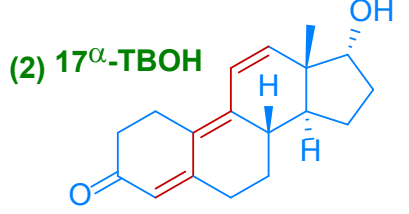

(3)

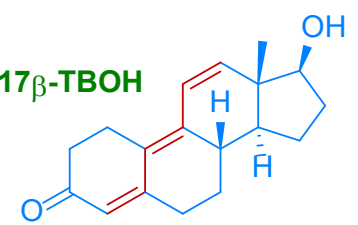<smiles>O=C1CCC2=C(CCC3CCCCC23)C(O)C=C1O</smiles>

(4) $12-\mathrm{OH}-\mathrm{TBOH}$

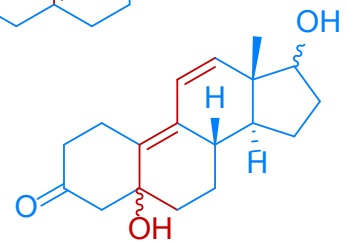

(5) $5-\mathrm{OH}-\mathrm{TBOH}$

TBA Metabolite Photohydrates

TBA Metabolites

Figure S1. Molecular structures of trenbolone acetate (TBA), its major known metabolites $17 \alpha-\mathrm{TBOH}$ and $17 \beta-\mathrm{TBOH}$, and the photohydrates of $17 \alpha-\mathrm{TBOH}$ and $17 \beta-\mathrm{TBOH}$ (i.e., $12-\mathrm{OH}-\mathrm{TBOH}$ and $5-\mathrm{OH}-$ $\mathrm{TBOH})$. Carbon atom position labelling is shown for TBA and is the same for the other structures provided. 


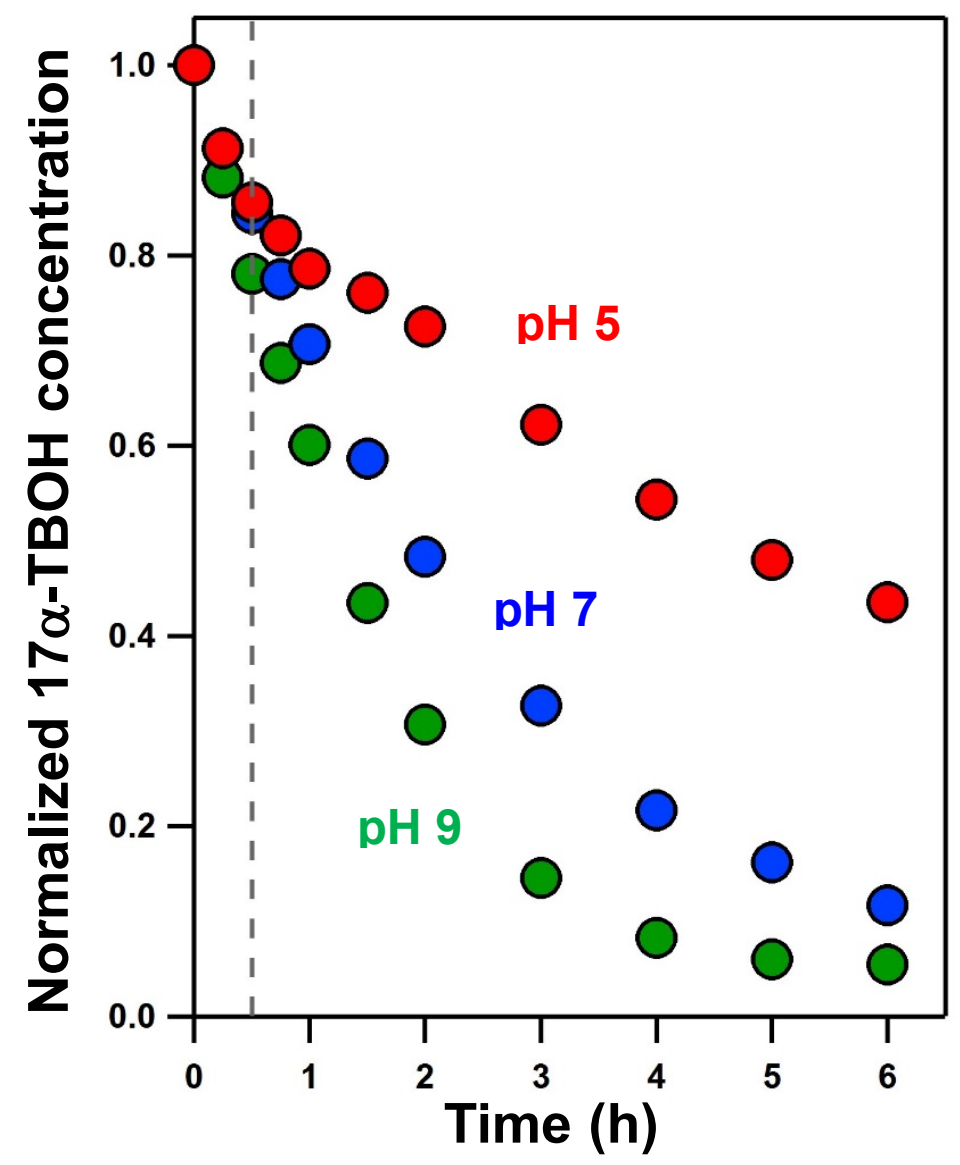

Figure S2. Photolysis of $17 \alpha-\mathrm{TBOH}$ as a function of $\mathrm{pH}$ (values provided). Dashed line denotes first 0.5 $\mathrm{h}$ of reaction, over which rates of $17 \alpha-\mathrm{TBOH}$ decay were nearly equivalent at all $\mathrm{pH}$ values, consistent with a $\mathrm{pH}$-independent photoreaction (which was assumed in our theoretical calculations of photohydration). Deviation in rates of $17 \alpha-\mathrm{TBOH}$ decay over longer timescales are assumed to reflect the influence of accumulated photohydrates, which over time can represent an appreciable source of $17 \alpha-$ $\mathrm{TBOH}$ in these systems. 

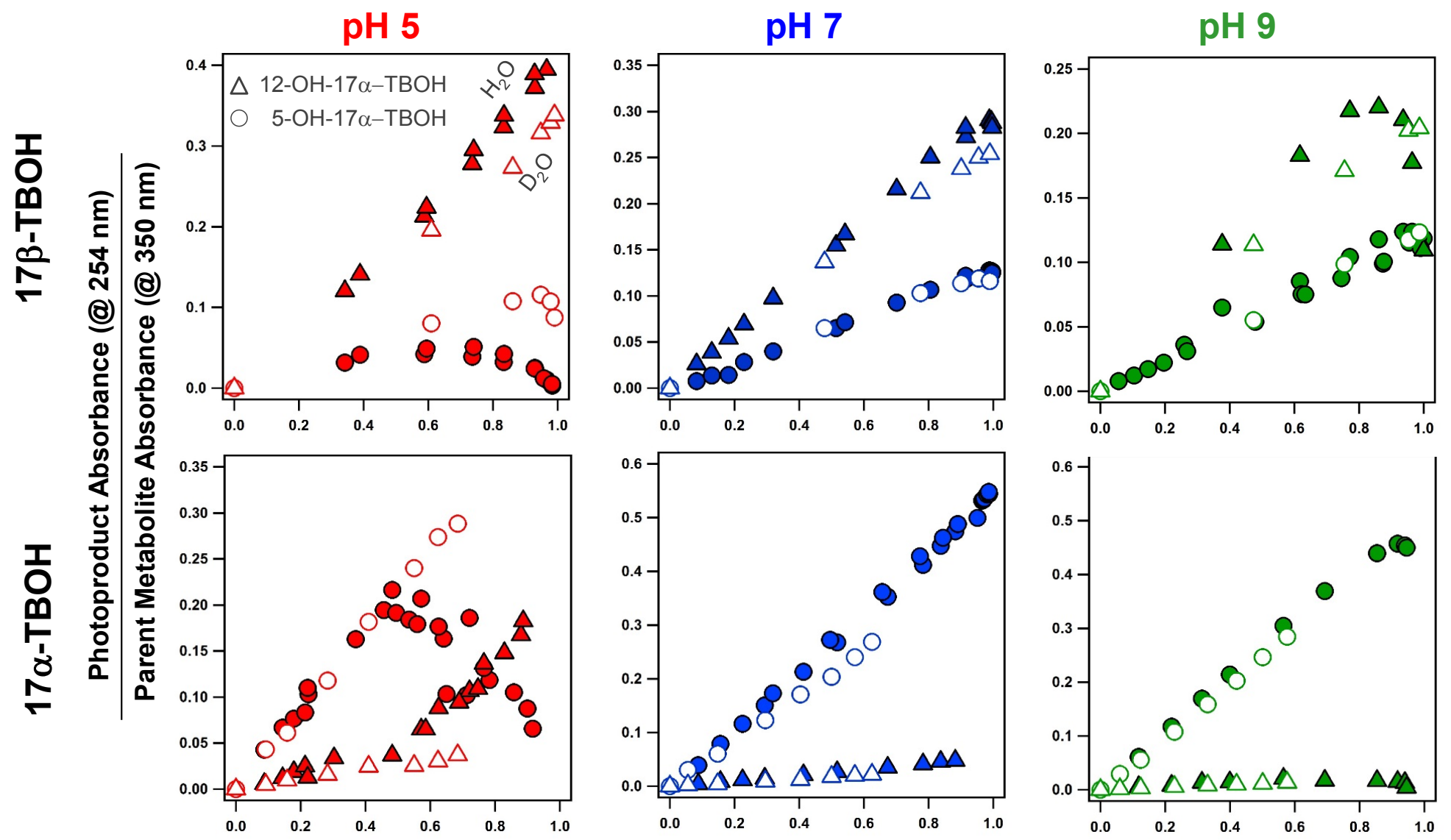

Fractional trenbolone conversion

Figure S3. Comparison of photohydrate yields from the direct photolysis of $17 \beta-\mathrm{TBOH}$ (top) and $17 \alpha-\mathrm{TBOH}$ (bottom) in $\mathrm{H}_{2} \mathrm{O}$ (solid symbols) and $\mathrm{D}_{2} \mathrm{O}$ (open symbols). Plots are analogous to Figure 2 in the main text, showing the peak area response for 5-OH- and 12-OH-TBOH (circles and triangles, respectively, as measured via DAD absorbance at $254 \mathrm{~nm}$ ) after normalization to the initial peak area of the corresponding parent TBA metabolite (measured via DAD absorbance at $350 \mathrm{~nm}$ ) as a function of reaction progress (expressed as fraction of parent TBA metabolite conversion). Data are shown for $\mathrm{pH} 5$ (red), $\mathrm{pH} 7$ (blue) and $\mathrm{pH} 9$ (green). 

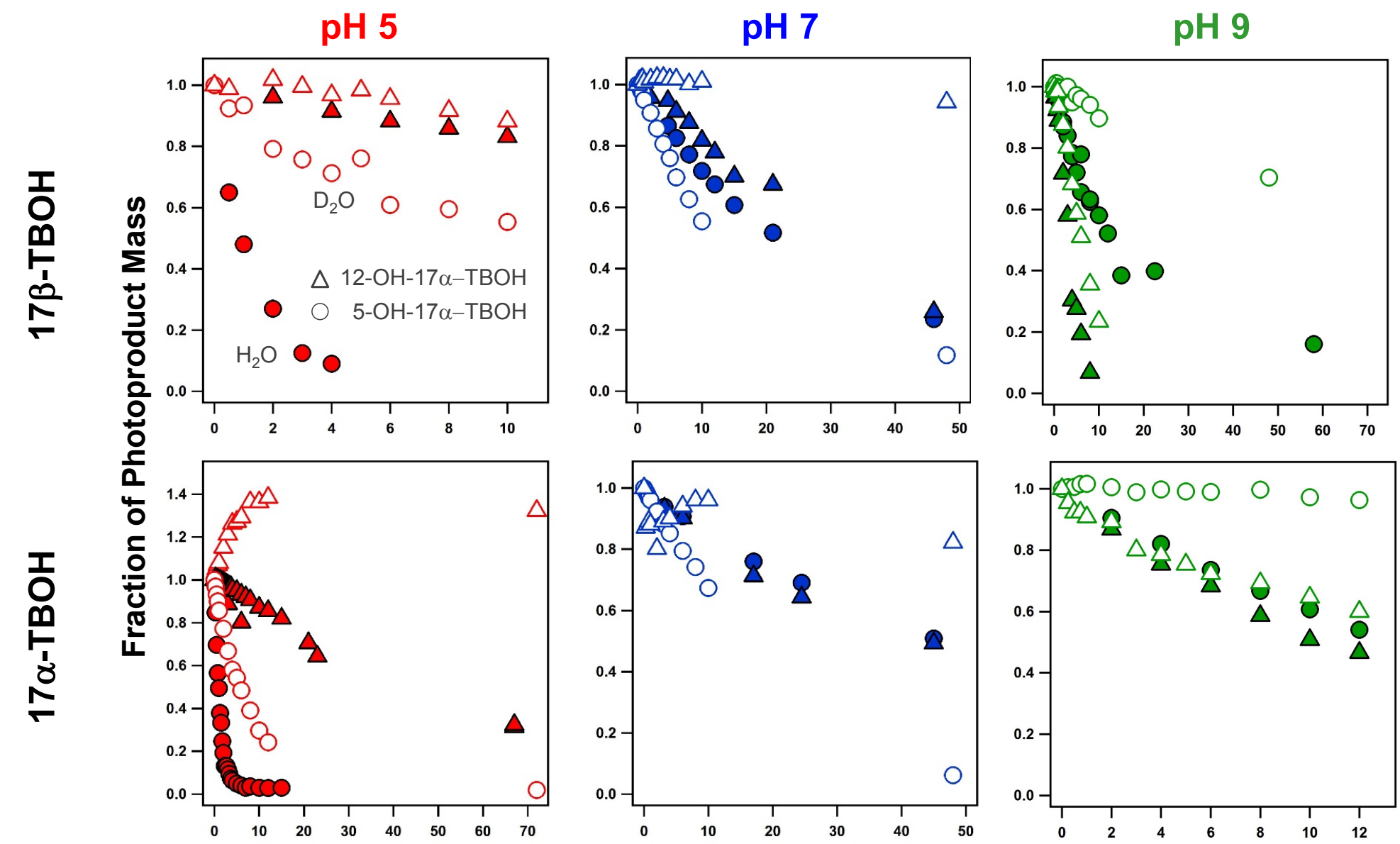

Time (dark in h)

Figure S4. Comparison of dark (thermal) decomposition of photohydrates of $17 \beta-\mathrm{TBOH}$ (top) and $17 \alpha-\mathrm{TBOH}$ (bottom) in $\mathrm{H}_{2} \mathrm{O}$ (solid symbols) and $\mathrm{D}_{2} \mathrm{O}$ (open symbols) at $\mathrm{pH} 5$ (red), 7 (blue) and 9 (green). Data are shown for both 12-OH-TBOH (triangles) and 5-OH-17TBOH (circles) and are analogous to Figure $4 \mathrm{a}$ and $4 \mathrm{~b}$ of the main text. All data has been normalized to the amount of photohydrate mass present at the conclusion of the photoreaction (based upon DAD absorbance at $254 \mathrm{~nm}$ ). 

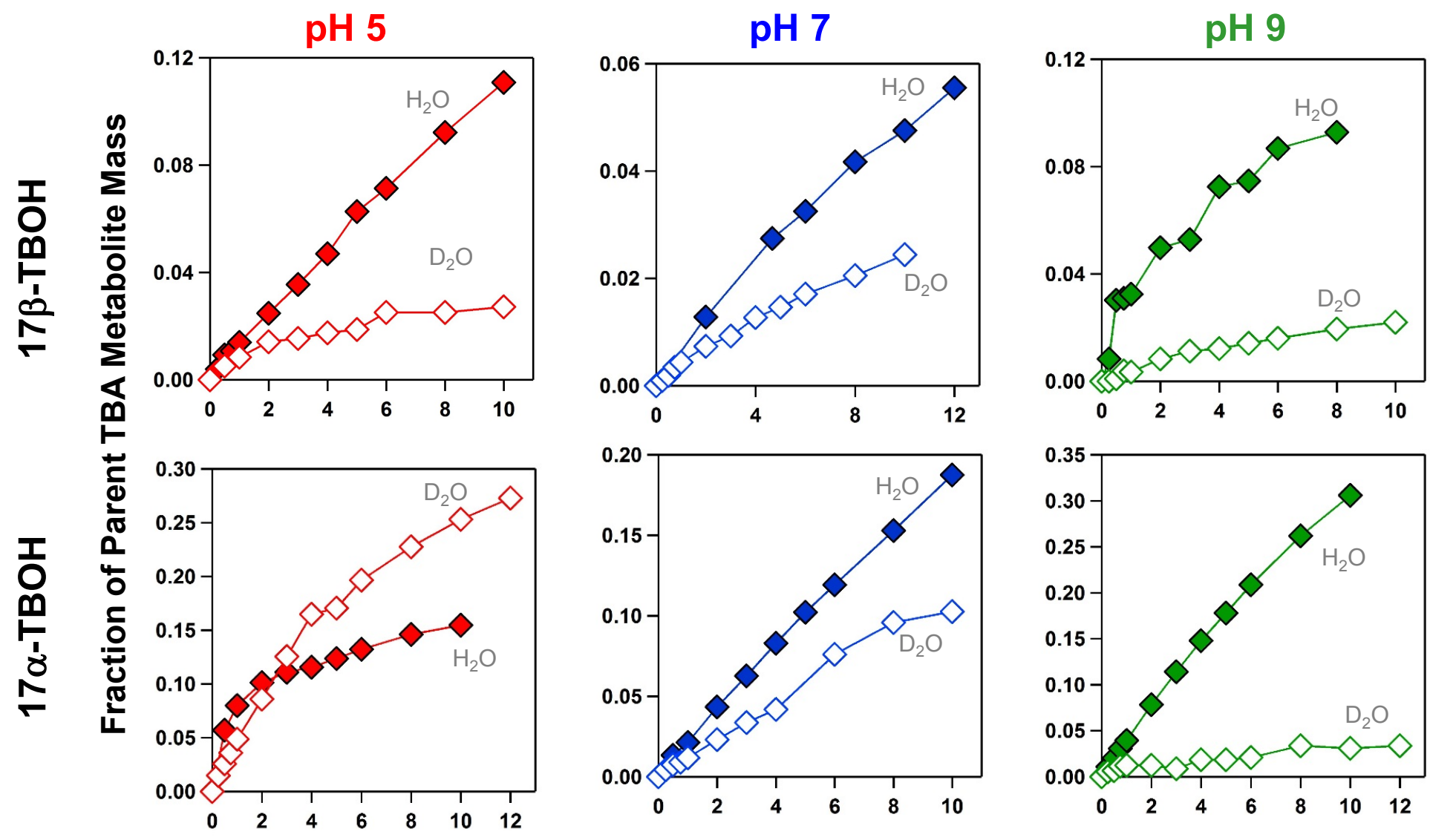

\section{Time (dark in h)}

Figure S5. Comparison of dark (thermal) regrowth of $17 \beta-\mathrm{TBOH}$ (top) and $17 \alpha-\mathrm{TBOH}$ (bottom) in $\mathrm{H}_{2} \mathrm{O}$ (solid symbols) and $\mathrm{D}_{2} \mathrm{O}$ (open symbols). Data are analogous to Figures $4 \mathrm{c}$ and $4 \mathrm{~d}$ of the main text, with reversion data presented as the fraction of the initial $17 \alpha-\mathrm{TBOH}$ and $17 \beta-\mathrm{TBOH}$ mass present in the system. 


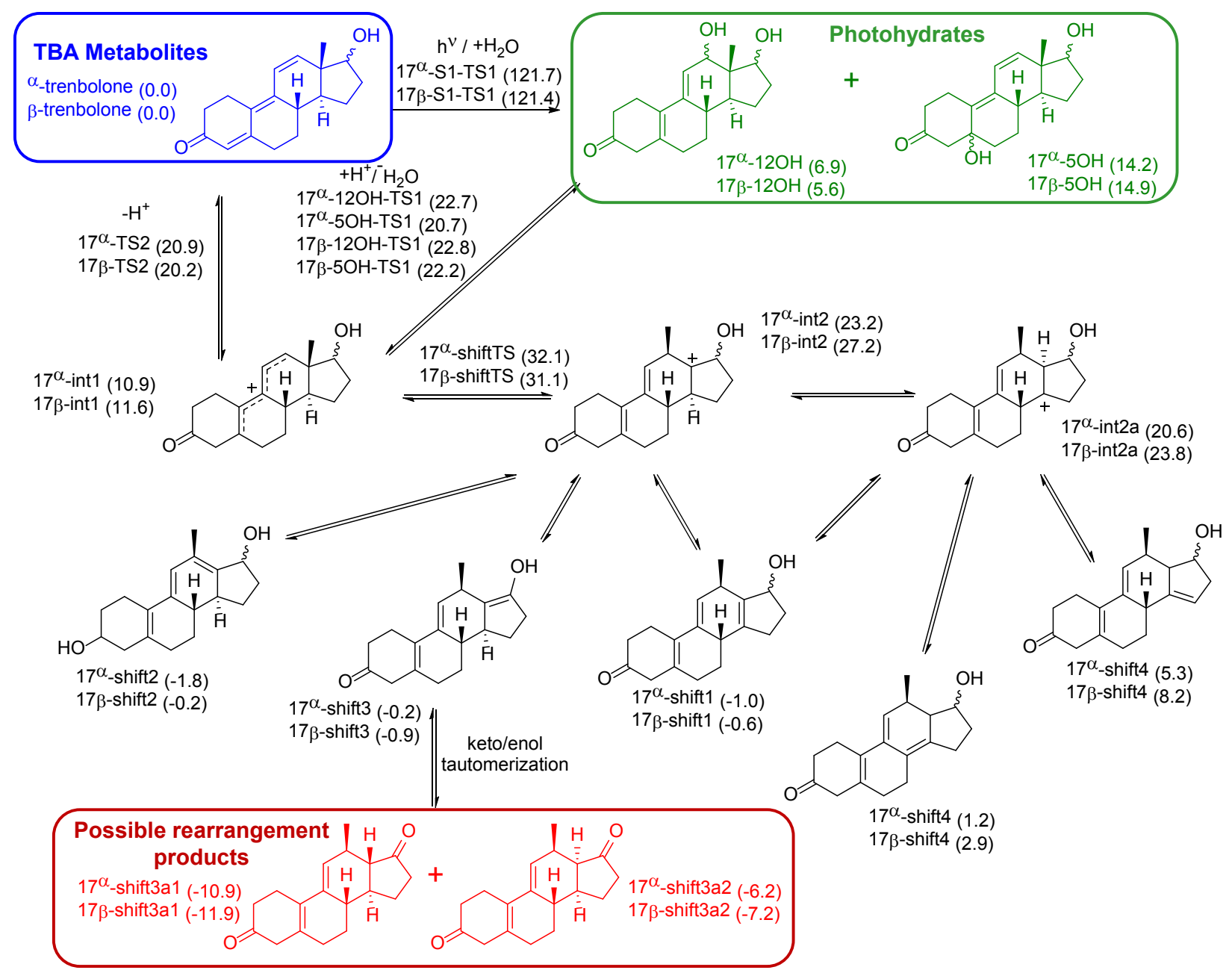

Figure S6. Alternative products and corresponding free energies $\left(\Delta \mathrm{G}_{298}\right)$ with respect to parent $17 \alpha$ TBOH and 17ß-TBOH calculated at M06-2X/6-311+G(2df,2p)//M062X/6-31+G(d,p) + SMD level of theory. 


\section{Additional Computational Details}

\section{Mechanistic Schemes}

Cartesian coordinates for these structures are found below.

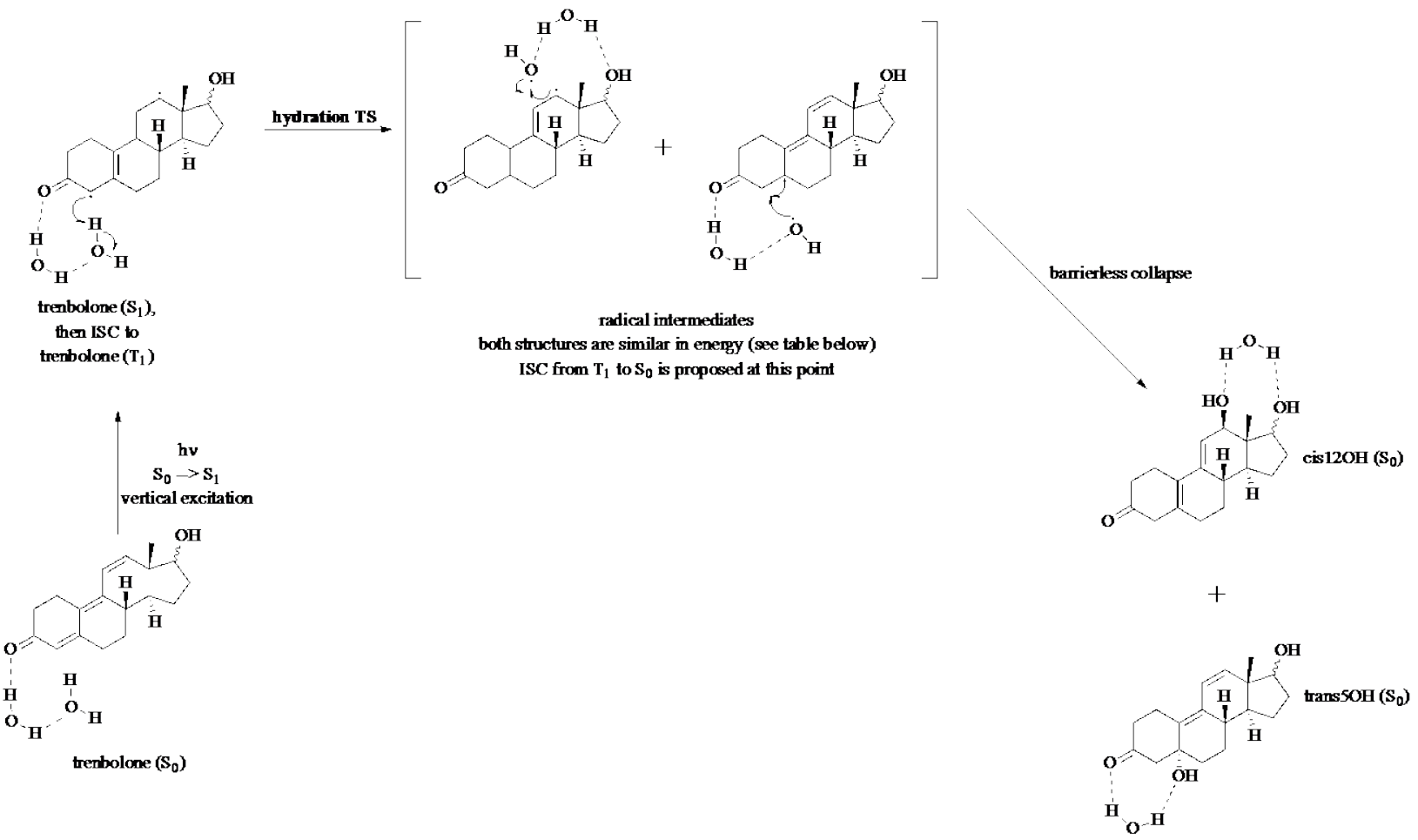

Scheme S1. Photochemical hydration pathway, showing approximate coordinated structures as used in the quantum chemical calculations. 


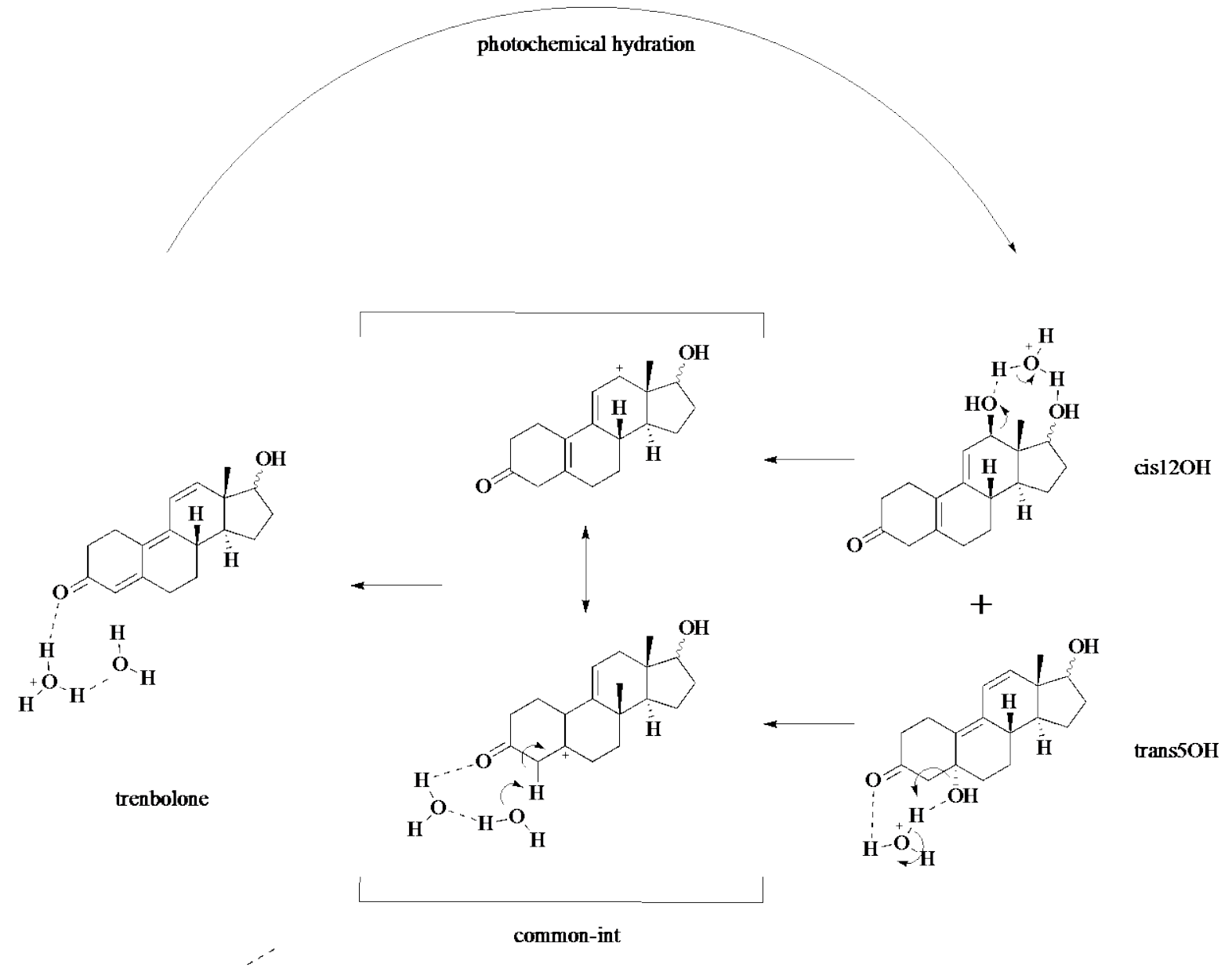

Scheme S2. Acidic reversion pathway ( $\mathrm{S}_{0}$ surface), showing approximate coordinated structures as used in the quantum chemical calculations. 


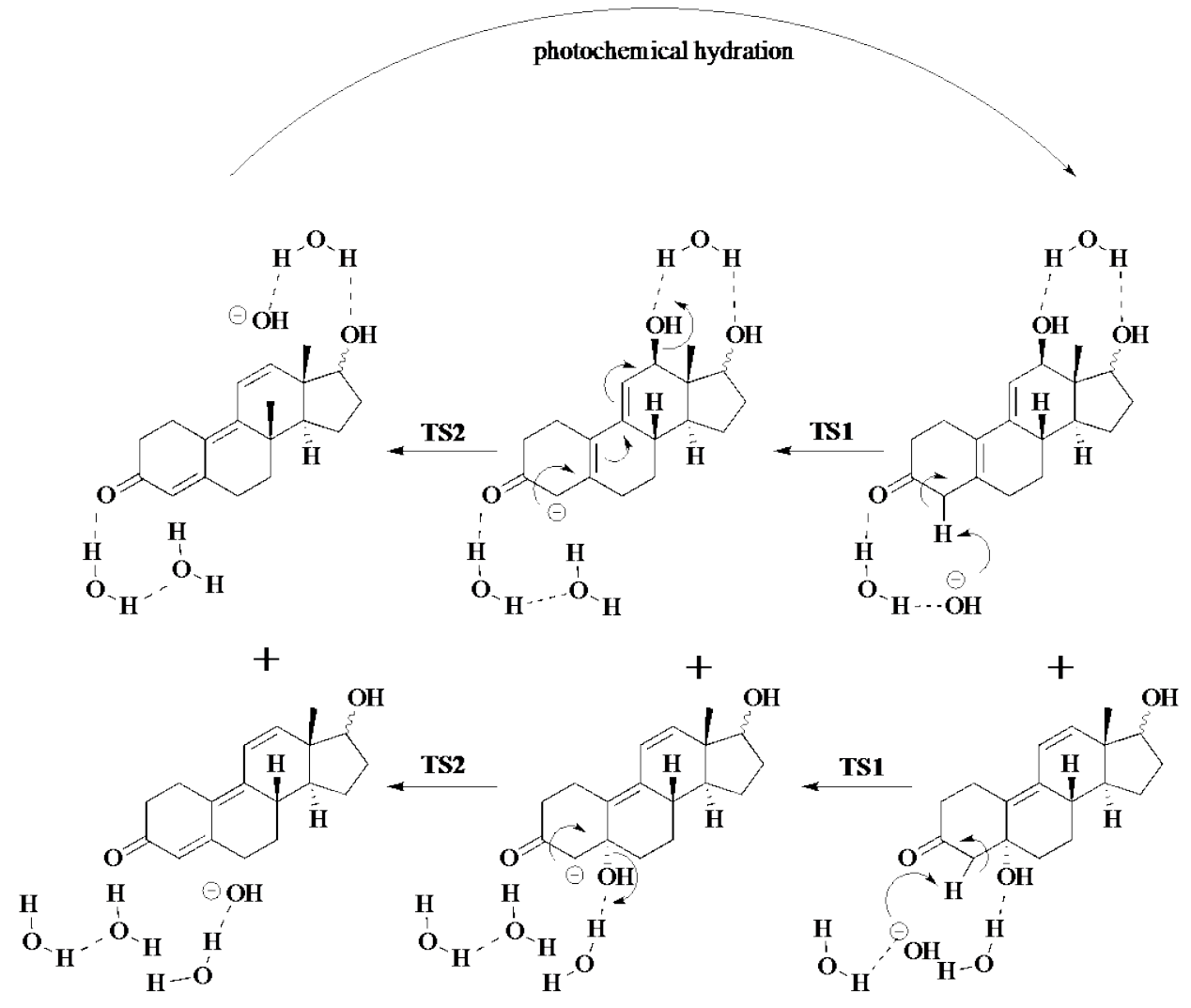

Scheme S3. Alkaline reversion pathway ( $\mathrm{S}_{0}$ surface), showing approximate coordinated structures as used in the quantum chemical calculations. 


\section{Cartesian Coordinates of Optimized Structures}

\section{Structures for neutral, photochemical hydration}

\section{$17 \alpha$}

trenbolone (SO) - complex with two waters near carbonyl

Charge $=0$ Multiplicity $=1$

C, $0,-0.7835518714,2.4486769138,-0.0238063583$

$\mathrm{C}, 0,-1.6832134657,1.274364154,-0.3037946823$

$\mathrm{C}, 0,-1.0886957051,-0.0455996272,-0.5291575696$

C, $0,0.2688273452,-0.2078217577,-0.5038382794$

C, $0,1.2063029554,0.9735846637,-0.2499652953$

$\mathrm{C}, 0,0.5267335705,2.0252641312,0.619924351$

$\mathrm{H}, 0,-0.5644755907,2.9290311003,-0.988451146$

$\mathrm{H}, 0,-1.3231121651,3.1829360676,0.5807878454$

$\mathrm{H}, 0,1.4378023455,1.4420875316,-1.2199718628$

$\mathrm{H}, 0,0.342217112,1.6063851197,1.6184101671$

$\mathrm{H}, 0,1.1841814272,2.8920330725,0.7391872083$

C,0,-2.0665149335,-1.1951983818,-0.6803356384

C,0,-3.0322967643,1.4709867541,-0.3378958822

C, $0,-3.959554933,0.4441480907,-0.7774405819$

C,0,-3.3559813082,-0.7872139691,-1.3919518232

$\mathrm{H}, 0,-1.6253651062,-2.0280990538,-1.2262354088$

$\mathrm{H}, 0,-4.0894330043,-1.59682299,-1.3830949481$

$\mathrm{H}, 0,-2.3229345863,-1.5678688613,0.321621399$

$\mathrm{H}, 0,-3.1435798793,-0.5376804452,-2.440847161$

$\mathrm{H}, 0,-2.9590828337,0.1882104055,1.9217626178$

C, $0,2.4985302451,0.4614985516,0.3683273068$

$\mathrm{H}, 0,2.222814079,-0.0356434684,1.3104060125$

$\mathrm{C}, 0,0.8841626667,-1.5310618845,-0.6909954524$

$\mathrm{H}, 0,0.2305047048,-2.3800138552,-0.8558861989$

C, $0,2.213962971,-1.7484267704,-0.6788507103$

$\mathrm{H}, 0,2.5993172329,-2.751870578,-0.853860248$

C, $0,3.1750476213,-0.6158469383,-0.4942631384$

C,0,4.4462673791,-0.8910656012,0.3217771905

$\mathrm{H}, 0,5.218202409,-1.3897537085,-0.2769284407$

C, $0,3.6331464545,1.4396761655,0.6795449533$

$\mathrm{H}, 0,3.7504859287,2.1652825039,-0.1337892019$

$\mathrm{H}, 0,3.454153562,2.0042033378,1.597903676$

C, $0,4.884045803,0.5204834085,0.7878380394$

$\mathrm{H}, 0,5.2592917933,0.4587049049,1.8137325572$

$\mathrm{H}, 0,5.7039473949,0.8902641335,0.1660802683$

C,0,3.608364247,-0.1111865445,-1.8860675122

$\mathrm{H}, 0,4.0891603975,-0.9269129345,-2.4358742526$

$\mathrm{H}, 0,2.7501946414,0.2263190531,-2.4724348642$

$\mathrm{H}, 0,4.3254809265,0.7127764835,-1.8144118707$

$\mathrm{O}, 0,4.0981603376,-1.7315543289,1.4262347923$

$\mathrm{H}, 0,4.881276364,-1.8393503934,1.9830690486$

$\mathrm{O}, 0,-5.1883677927,0.5953638752,-0.6973031442$

$\mathrm{H}, 0,-3.4526621228,2.4380483317,-0.0728248191$

O,0,-3.4812379882,-0.3406963697,2.5405799682 
$\mathrm{H}, 0,-4.1331407428,-0.8117825918,1.9855009401$

$\mathrm{O}, 0,-5.458862887,-1.7102122837,1.0288427342$

$\mathrm{H}, 0,-6.2452120744,-1.829558231,1.5777729994$

$\mathrm{H}, 0,-5.7005646195,-1.0370447215,0.3707373568$

trenbolone (T1) - complex with two waters near carbonyl

Charge $=0$ Multiplicity $=3$

C, $0,-0.7689152383,2.4329803784,-0.2213599771$

C, $0,-1.6538897752,1.2190229016,-0.3867235291$

C, $0,-1.1332723289,-0.0617721087,-0.439143828$

C, $0,0.3250529245,-0.2944682142,-0.3316474704$

C, $0,1.233962298,0.9217823433,-0.3601122777$

$\mathrm{C}, 0,0.5797055513,2.0831665302,0.3928560912$

$\mathrm{H}, 0,-0.6156128158,2.8942091723,-1.2065039546$

$\mathrm{H}, 0,-1.2936911172,3.1746687675,0.389597631$

$\mathrm{H}, 0,1.3641218932,1.2413773709,-1.4090051852$

$\mathrm{H}, 0,0.4474519357,1.7950441337,1.4445538775$

$\mathrm{H}, 0,1.240249684,2.9560634668,0.3715721238$

C,0,-2.0607021073,-1.2447061105,-0.5374105415

C,0,-3.0613660352,1.4273782262,-0.4252994609

C,0,-3.9891036199,0.3863170443,-0.7332211917

C,0,-3.4055166299,-0.9232809593,-1.1889043961

$\mathrm{H}, 0,-1.5862150673,-2.051727019,-1.0990182052$

$\mathrm{H}, 0,-4.1273831557,-1.7262364843,-1.0222909828$

$\mathrm{H}, 0,-2.2218802947,-1.6326551481,0.4803289045$

$\mathrm{H}, 0,-3.266215231,-0.8276900143,-2.2752739643$

$\mathrm{H}, 0,-2.9894909907,0.0053107087,1.9911915808$

C, $0,2.5897400757,0.5546497064,0.2266869723$

$\mathrm{H}, 0,2.4190147561,0.325642457,1.2894469595$

C, $0,0.8405118578,-1.5706162233,-0.1710022038$

$\mathrm{H}, 0,0.1599005053,-2.4121458371,-0.0800049247$

C, $0,2.2200698994,-1.8506475175,-0.1377466118$

$\mathrm{H}, 0,2.5705574493,-2.8716604944,-0.0225076157$

$\mathrm{C}, 0,3.1707769173,-0.730381586,-0.395526584$

C, $0,4.5497245064,-0.7937400441,0.2736231883$

$\mathrm{H}, 0,5.2385665177,-1.4529790943,-0.2689588604$

C, $0,3.7440418599,1.5586443328,0.1412110396$

$\mathrm{H}, 0,3.7273252463,2.0804734726,-0.8225603101$

$\mathrm{H}, 0,3.6858328044,2.319062903,0.923795942$

C, $0,5.0211549876,0.6795621354,0.2712076856$

$\mathrm{H}, 0,5.5731105608,0.8843831322,1.1935554974$

$\mathrm{H}, 0,5.708384986,0.8555844653,-0.5609854322$

C, $0,3.3797783311,-0.6062997456,-1.9208607447$

$\mathrm{H}, 0,3.8508260161,-1.5177983343,-2.3024047694$

$\mathrm{H}, 0,2.425796311,-0.4783450612,-2.4393763471$

$\mathrm{H}, 0,4.0253753774,0.2409777646,-2.1746186392$

$\mathrm{O}, 0,4.3792576283,-1.2893311945,1.6050983685$

$\mathrm{H}, 0,5.2320922353,-1.2335421234,2.0569078768$

$0,0,-5.229979409,0.5645269491,-0.6815284934$

$\mathrm{H}, 0,-3.4632477366,2.4150892274,-0.2138103683$ 
O,0,-3.6418750078,-0.3488399137,2.6099890529 $\mathrm{H}, 0,-4.3349917797,-0.7580502441,2.0558449327$ $\mathrm{O}, 0,-5.7830162197,-1.5211265924,1.1542624608$ $\mathrm{H}, 0,-6.581004411,-1.4011978529,1.6855183417$ $\mathrm{H}, 0,-5.8486456275,-0.8684244644,0.4335046521$

hydration TS1 (common) (T1)

Charge $=0$ Multiplicity $=3$

C, $0,-0.8755376936,2.2749934695,-0.2595020978$

C, $0,-1.6780187487,1.0037374066,-0.2918144386$

C, $0,-1.1209288507,-0.2385850325,-0.1916097699$

C, $0,0.3193252886,-0.4067517975,-0.1315119796$

C, $0,1.1815357241,0.8297450607,-0.3353690018$

C, $0,0.5207917749,2.0511871226,0.3077297317$

$\mathrm{H}, 0,-0.8102872803,2.6844295702,-1.2788307493$

$\mathrm{H}, 0,-1.4171033409,3.0235891032,0.3318102341$

$\mathrm{H}, 0,1.2551482691,1.028801281,-1.4189670475$

$\mathrm{H}, 0,0.4559593748,1.8856648547,1.3916659562$

$\mathrm{H}, 0,1.14269996,2.9380969352,0.1471623384$

C,0,-2.0545046442,-1.4335070397,-0.1620831293

C,0,-3.1633310646,1.1646117874,-0.3620252932

C,0,-3.9424562004,0.0287736431,-0.9609142625

C,0,-3.2099276669,-1.2653092428,-1.1529581725

$\mathrm{H}, 0,-1.5286977129,-2.3541984533,-0.414446254$

$\mathrm{H}, 0,-3.9243140095,-2.0909111401,-1.1039236288$

$\mathrm{H}, 0,-2.4591803091,-1.5627286448,0.8510212537$

$\mathrm{H}, 0,-2.806916921,-1.2326431899,-2.1754719956$

$\mathrm{H}, 0,-3.5567413861,1.2125448754,0.7373435409$

C, $0,2.5751674628,0.5770453786,0.2203841524$

$\mathrm{H}, 0,2.460659275,0.4417772596,1.306593727$

C,0,0.9284526417,-1.6595311231,0.1195527643

$\mathrm{H}, 0,0.2943735548,-2.512075059,0.345357456$

C, $0,2.2913017614,-1.8668599783,0.0870091441$

$\mathrm{H}, 0,2.7014719049,-2.8566122699,0.2710295714$

C, $0,3.1874501256,-0.734967747,-0.3060425031$

C,0,4.5895541962,-0.6821430109,0.3156175744

$\mathrm{H}, 0,5.2892792051,-1.3559079651,-0.1945010666$

C, $0,3.6787671732,1.6141602766,-0.0070771726$

$\mathrm{H}, 0,3.6111482496,2.0302811268,-1.0190330215$

$\mathrm{H}, 0,3.6105492151,2.4498648918,0.6937897181$

C, $0,4.9953172225,0.8047157861,0.1701252079$

$\mathrm{H}, 0,5.5538976176,1.1143792624,1.0587197227$

$\mathrm{H}, 0,5.6593259773,0.9347898668,-0.6889589466$

C,0,3.3496142251,-0.7421522963,-1.8412336814

$\mathrm{H}, 0,3.8295871198,-1.6749096117,-2.1547909129$

$\mathrm{H}, 0,2.3786282868,-0.6796066634,-2.339736561$

$\mathrm{H}, 0,3.9695473175,0.0901367193,-2.1910357169$

$\mathrm{O}, 0,4.489220316,-1.0704324527,1.689840339$

$\mathrm{H}, 0,5.3506309777,-0.931609115,2.1067684423$

$\mathrm{O}, 0,-5.1251119969,0.1670362546,-1.2543926755$ 
$\mathrm{H}, 0,-3.4863397917,2.1215260753,-0.7837006241$ $\mathrm{O}, 0,-4.0273451158,0.9681481383,2.1153205247$ $\mathrm{H}, 0,-4.5337592777,0.1408842773,1.9104725899$ $\mathrm{O}, 0,-5.4044225608,-1.343507911,1.3374534487$ $\mathrm{H}, 0,-6.1028040801,-1.6007197296,1.9546792169$ $\mathrm{H}, 0,-5.8592293701,-1.1245516917,0.5105351508$

diradical intermediate (common) (T1)

Charge $=0$ Multiplicity $=3$

C,0,-1.1199316691,2.2727808794,-0.1365178864

C, $0,-1.9080417502,1.0144280645,-0.3745045249$

C, $0,-1.319579519,-0.2221159168,-0.4652257888$

C, $0,0.1156487571,-0.3665140319,-0.3773176139$

C, $0,0.9707703188,0.8901050226,-0.3527473571$

C, $0,0.2704366425,2.0036883311,0.4265069624$

$\mathrm{H}, 0,-1.0412099319,2.8184256956,-1.089568975$

$\mathrm{H}, 0,-1.6874500691,2.9270271235,0.5370727601$

$\mathrm{H}, 0,1.0942929324,1.2439054518,-1.3914802318$

$\mathrm{H}, 0,0.1912874599,1.7010346233,1.4783357475$

$\mathrm{H}, 0,0.8733364909,2.9172909071,0.39168133$

C,0,-2.1795861218,-1.4487761964,-0.7212217098

C, $0,-3.3967231396,1.216405775,-0.4480415252$

C,0,-4.2031284301,-0.0088651892,-0.7830977612

C, $0,-3.4681678471,-1.107035991,-1.4822672845$

$\mathrm{H}, 0,-1.6273001094,-2.1759986805,-1.3194261284$

$\mathrm{H}, 0,-4.1183440887,-1.9770414131,-1.5931101455$

$\mathrm{H}, 0,-2.4288937984,-1.9440825129,0.2259974008$

$\mathrm{H}, 0,-3.2011836122,-0.7322606979,-2.4797625676$

$\mathrm{H}, 0,-3.7755286133,1.6340780501,0.4952087216$

C,0,2.3397978193,0.561421212,0.2243315929

$\mathrm{H}, 0,2.1746241144,0.2539564141,1.268172127$

C,0,0.7365556289,-1.644509079,-0.3084415355

$\mathrm{H}, 0,0.1018373274,-2.5244966137,-0.2490245345$

C,0,2.0968582054,-1.8328280692,-0.313204301

$\mathrm{H}, 0,2.5141129239,-2.8360816427,-0.2712516742$

C,0,2.9955615784,-0.6460521539,-0.4727968132

C,0,4.3636906657,-0.6854559758,0.2216032672

$\mathrm{H}, 0,5.0982074916,-1.2635131543,-0.3530004868$

C, $0,3.4384885658,1.6279946954,0.2202075766$

$\mathrm{H}, 0,3.417101788,2.1971755341,-0.7165656909$

$\mathrm{H}, 0,3.3237290654,2.3435177155,1.0382569152$

C, $0,4.7551189808,0.8078278525,0.3377103121$

$\mathrm{H}, 0,5.2611923738,0.9757393854,1.2932959546$

$\mathrm{H}, 0,5.4619243094,1.0780761203,-0.4517497821$

C, $0,3.236393723,-0.4073954348,-1.9785990375$

$\mathrm{H}, 0,3.7440433888,-1.2762845812,-2.4101231491$

$\mathrm{H}, 0,2.2913733532,-0.2720859716,-2.5113136649$

$\mathrm{H}, 0,3.8625985854,0.4726552295,-2.1597261432$

O,0,4.2012427022,-1.2907961292,1.5087691581

$\mathrm{H}, 0,5.039640627,-1.2173039465,1.9849608253$ 
$0,0,-5.3918874008,-0.0824923091,-0.4899404173$

$\mathrm{H}, 0,-3.6319992546,1.9681550279,-1.218942655$

$0,0,-1.6208216392,-0.0880379077,2.2249730817$

$\mathrm{H}, 0,-2.5621910838,-0.318917897,2.0257946043$

$\mathrm{O}, 0,-4.317174895,-0.8611152807,2.1550677311$

$\mathrm{H}, 0,-4.5553425921,-0.7100445736,3.0800273768$

$\mathrm{H}, 0,-4.9842272538,-0.3878097611,1.6365069403$

diradical intermediate cis-12OH (T1)

Charge $=0$ Multiplicity $=3$

C, $0,-2.5316620794,-2.1508046683,0.1193017086$

C, $0,-3.0364248815,-0.7355922557,0.0354428767$

C, $0,-2.1945243974,0.3439845694,-0.0016983562$

C, $0,-0.7642707465,0.1692043804,0.0546153534$

C, $0,-0.2062351281,-1.2243074324,0.3013753018$

$\mathrm{C}, 0,-1.0912920964,-2.2805417919,-0.3598817743$

$\mathrm{H}, 0,-2.6124080779,-2.4950904375,1.1619787577$

$\mathrm{H}, 0,-3.1921835773,-2.8025676248,-0.4649689142$

$\mathrm{H}, 0,-0.2206963602,-1.4185141594,1.3881448683$

$\mathrm{H}, 0,-1.0515362372,-2.1441760441,-1.4492126905$

$\mathrm{H}, 0,-0.7068431313,-3.2820890585,-0.1396112068$

C, $0,-2.7680914165,1.748172039,-0.0977552165$

C,0,-4.5355633107,-0.6144169299,-0.0397657449

$\mathrm{C}, 0,-5.0685140159,0.7909210313,-0.1085255792$

$\mathrm{C}, 0,-4.1861128836,1.8471281084,0.4792777972$

$\mathrm{H}, 0,-2.1398795355,2.4494756104,0.455278973$

$\mathrm{H}, 0,-4.6239224498,2.8335539568,0.3137926911$

$\mathrm{H}, 0,-2.7697076226,2.0857422508,-1.141769285$

$\mathrm{H}, 0,-4.1416760091,1.6557420119,1.5601395015$

$\mathrm{H}, 0,-4.9269255838,-1.18013987,-0.8944679668$

C, $0,1.2315370359,-1.2896389409,-0.1933155244$

$\mathrm{H}, 0,1.1965066579,-1.1254690692,-1.2809514991$

$\mathrm{C}, 0,0.1330893151,1.2591705364,-0.1320853227$

$\mathrm{H}, 0,-0.281688573,2.2339294151,-0.3728006151$

C, $0,1.4976010492,1.1566087213,-0.0246780681$

$\mathrm{H}, 0,2.126200252,2.0338942746,-0.1599159118$

C,0,2.1032170469,-0.1534338842,0.3759424272

C, $0,3.4777117588,-0.5185763125,-0.2022495053$

C, $0,2.062733341,-2.5503581868,0.0585929239$

$\mathrm{H}, 0,1.8728186511,-2.9414662083,1.0649340393$

$\mathrm{H}, 0,1.8300645174,-3.3486235899,-0.6504681123$

C, $0,3.532274947,-2.0587980308,-0.0745485474$

$\mathrm{H}, 0,4.0302847056,-2.4816075582,-0.9526765047$

$\mathrm{H}, 0,4.1273745133,-2.3421901855,0.7975934284$

C, $0,2.2168092463,-0.1974852737,1.9142292975$

$\mathrm{H}, 0,2.8712801086,0.6122727041,2.2558354403$

$\mathrm{H}, 0,1.2395725943,-0.0588246043,2.3842377976$

$\mathrm{H}, 0,2.6393588203,-1.1434669868,2.269085351$

$\mathrm{O}, 0,-6.1643210923,1.0337492816,-0.5994741913$

$\mathrm{H}, 0,-4.9878846985,-1.0769608687,0.8529419335$ 
$\mathrm{O}, 0,4.3416748621,2.3966274862,1.4814967389$

$\mathrm{H}, 0,4.3785111349,2.401886821,0.4811527624$

$0,0,4.5014989608,2.4427680095,-1.2298360437$

$\mathrm{H}, 0,4.1009711521,1.5815714524,-1.4815365551$

$\mathrm{H}, 0,3.8797165793,3.1165560833,-1.5368282889$

$\mathrm{H}, 0,4.2974721854,-0.023826993,0.3353400065$

$0,0,3.518064448,-0.1023529603,-1.5781879343$

$\mathrm{H}, 0,4.2561858483,-0.553359933,-2.0128808403$

hydration TS2 cis- $12 \mathrm{OH}(\mathrm{T} 1)$ - complexed water and hydroxyl radical at $\mathrm{C}_{17}$

Charge $=0$ Multiplicity $=3$

C, $0,-2.3600818485,-2.2574073049,-0.0502544289$

$\mathrm{C}, 0,-2.8913006466,-0.8676901346,-0.2107763962$

C, $0,-2.0296968798,0.2485183171,-0.3783052379$

C, $0,-0.6572519104,0.1047175544,-0.2566774793$

C, $0,-0.0772269546,-1.2478838198,0.145840056$

C, $0,-0.8994436484,-2.3727432814,-0.4843037061$

$\mathrm{H}, 0,-2.4557950146,-2.5538142994,1.0070477649$

$\mathrm{H}, 0,-2.9948054989,-2.9535087972,-0.6127188752$

$\mathrm{H}, 0,-0.1690349373,-1.3586362818,1.2389529426$

$\mathrm{H}, 0,-0.8268358871,-2.3025386046,-1.5776256255$

$\mathrm{H}, 0,-0.4965332187,-3.3463535321,-0.1863925283$

C,0,-2.7108116758,1.5677610093,-0.6748619032

C,0,-4.3760614178,-0.6935144563,-0.2902087716

C, $0,-4.8871179749,0.6407242441,0.2050429508$

C, $0,-3.9023059757,1.7758200824,0.2659955313$

$\mathrm{H}, 0,-2.0257994351,2.4076359135,-0.5617806499$

$\mathrm{H}, 0,-4.4297981224,2.7109240316,0.0618890019$

$\mathrm{H}, 0,-3.0682786755,1.5703500246,-1.7126125627$

$\mathrm{H}, 0,-3.5479798407,1.8180489785,1.3054521829$

$\mathrm{H}, 0,-4.7053711616,-0.7618578617,-1.3433011435$

C,0,1.4033742621,-1.3046327124,-0.2080573763

$\mathrm{H}, 0,1.4934228153,-1.2531171239,-1.3037741197$

C,0,0.2693099298,1.1702696274,-0.6471566336

$\mathrm{H}, 0,-0.1128615053,2.0011845219,-1.2329205828$

C,0,1.6236645559,1.1271542431,-0.3879773955

$\mathrm{H}, 0,2.2793486681,1.7070426395,-1.029174643$

C, $0,2.1521432405,-0.069544097,0.3607265027$

C, $0,3.6227828666,-0.4436194977,0.1265170826$

C, $0,2.2217829913,-2.5013375342,0.2854346886$

$\mathrm{H}, 0,1.8597367518,-2.833363286,1.2650854567$

$\mathrm{H}, 0,2.1455265643,-3.354036107,-0.3936747921$

C,0,3.675387039,-1.9651554686,0.3903880025

$\mathrm{H}, 0,4.3439255217,-2.4370578542,-0.3362218019$

$\mathrm{H}, 0,4.0944466177,-2.1570942198,1.3818671717$

C, $0,1.9384629287,0.0623845518,1.8794110426$

$\mathrm{H}, 0,2.6294732117,0.8024048091,2.2898603479$

$\mathrm{H}, 0,0.9222877104,0.3901460841,2.1133843541$

$\mathrm{H}, 0,2.1159839376,-0.8915148514,2.3867629854$

O,0,-6.0564294123,0.7781851649,0.5380704645 
$\mathrm{H}, 0,-4.904749435,-1.4928674199,0.2391904306$ $\mathrm{O}, 0,1.7840325319,2.8756983158,0.8237343659$ $\mathrm{H}, 0,2.7303990515,2.8816692849,0.5828982394$ $\mathrm{O}, 0,4.5855789727,2.5833696791,-0.3342002617$ $\mathrm{H}, 0,4.4763522807,1.7399502359,-0.8101377278$ $\mathrm{H}, 0,4.5327605577,3.2657276899,-1.0165809866$ $\mathrm{H}, 0,4.2908073008,0.108650231,0.7993266012$ $\mathrm{O}, 0,3.9720219291,-0.1139841759,-1.225966934$ $\mathrm{H}, 0,4.8212648402,-0.5307145118,-1.4308976033$

diol cis- $12 \mathrm{OH}(\mathrm{T} 1)$ - water complexed between $\mathrm{C}_{12}$ and $\mathrm{C}_{17}$ Charge $=0$ Multiplicity $=3$

C,0,2.3583652578,2.2679441586,0.087741891

C, $0,2.8770020138,0.8693919084,-0.0710039732$ C, $0,2.0253936542,-0.2250069167,0.1295979552$ C, $0,0.6914168505,-0.0399998592,0.4883659894$ C, $0,0.0371792266,1.3343639446,0.4718632279$ C, $0,0.882624261,2.3552819692,-0.2931803776$ $\mathrm{H}, 0,2.4940291713,2.5903290182,1.1332159103$ $\mathrm{H}, 0,2.9541256205,2.9561925859,-0.523188974$ $\mathrm{H}, 0,-0.0968101722,1.6879588051,1.5084452584$ $\mathrm{H}, 0,0.783872272,2.1534632067,-1.3687826704$ $\mathrm{H}, 0,0.4982399091,3.3651415035,-0.1156132394$ C, $0,2.5951297275,-1.6254968694,-0.000082716$ C, $0,4.3351153706,0.7200157078,-0.3900805328$ C, $0,4.8733506439,-0.6886593043,-0.3490820474$ C,0,4.0676685597,-1.6896741022,0.4247276829 $\mathrm{H}, 0,2.0163424187,-2.3263747506,0.6066160507$ $\mathrm{H}, 0,4.4930362577,-2.6859036328,0.287036425$ $\mathrm{H}, 0,2.5038747888,-1.9635598064,-1.0401042272$ $\mathrm{H}, 0,4.1474939732,-1.4146534206,1.4855609513$ $\mathrm{H}, 0,4.5795237054,1.1550960837,-1.3692003023$ C,0,-1.3447599245,1.181169048,-0.1747025702 $\mathrm{H}, 0,-1.1625134676,0.8398864523,-1.2058165458$ C,0,-0.1837848642,-1.1579516503,0.9150400208 $\mathrm{H}, 0,-0.0870670568,-1.5385555314,1.9331538511$ C, $0,-1.5504022061,-1.2655514853,0.3098198269$ $\mathrm{H}, 0,-1.4652417805,-1.4622028168,-0.7709013306$ C, $0,-2.2418117465,0.1105248871,0.4807557505$ $\mathrm{C}, 0,-3.5601818985,0.3248459444,-0.2843753525$ C, $0,-2.2434197844,2.425234605,-0.2458357065$ $\mathrm{H}, 0,-2.1360361069,3.0237516529,0.6660936898$ $\mathrm{H}, 0,-1.9797059945,3.0692778803,-1.0885251724$ C, $0,-3.685303708,1.8605540816,-0.3658332728$ $\mathrm{H}, 0,-4.1717989559,2.1444631238,-1.304442388$ $\mathrm{H}, 0,-4.3213970725,2.2248384815,0.4452639496$ C, $0,-2.5114597377,0.3908581732,1.9659883138$ $\mathrm{H}, 0,-3.2485071897,-0.3165633232,2.3574364085$ $\mathrm{H}, 0,-1.6034753224,0.2941110737,2.5668623777$ $\mathrm{H}, 0,-2.9041460133,1.4000626653,2.1200999468$ 
$0,0,5.9254388856,-0.9759575101,-0.9070317969$ $\mathrm{H}, 0,4.9337630803,1.2940733486,0.3371262519$ $\mathrm{O}, 0,-2.2533194596,-2.3318048313,0.9360501571$ $\mathrm{H}, 0,-2.9860537605,-2.593503992,0.3442154693$ O,0,-4.1682987717,-2.8520063846,-1.0320741449 $\mathrm{H}, 0,-3.9427307313,-1.974713256,-1.4069218881$ $\mathrm{H}, 0,-3.7925064756,-3.5005898879,-1.6416089905$ $\mathrm{H}, 0,-4.4134827822,-0.1404477357,0.2251308203$ $\mathrm{O}, 0,-3.4437185159,-0.2482168931,-1.598488442$ $\mathrm{H}, 0,-4.1490241493,0.1180876503,-2.1511455147$

diol cis-12OH (SO) - water complexed between $\mathrm{C}_{12}$ and $\mathrm{C}_{17}$ Charge $=0$ Multiplicity $=1$

C, $0,2.302516214,2.2273028095,-0.3018673987$ C, $0,2.8769896581,0.8565958572,-0.04607526$ C, $0,2.1161734077,-0.2485573276,0.1034410966$ C, $0,0.6374315344,-0.1651352892,0.0288184578$ C, $0,0.0299210247,1.2287597323,0.0663763188$ C, $0,0.8621103935,2.1786695027,-0.7949003338$ $\mathrm{H}, 0,2.3632613695,2.8090984133,0.6298917166$ $\mathrm{H}, 0,2.9392617792,2.7505717391,-1.0249992634$ $\mathrm{H}, 0,0.0850588502,1.5918135968,1.1067238885$ $\mathrm{H}, 0,0.8375058544,1.8262403234,-1.8349803511$ $\mathrm{H}, 0,0.4217073103,3.1815101761,-0.7791048082$ C,0,2.7481527891,-1.602902146,0.3528762092 C,0,4.3849531818,0.8252120567,-0.0014047411 $\mathrm{C}, 0,4.9971127374,-0.5216336283,0.27534558$ C,0,4.1291561091,-1.495328709,1.0105010006 $\mathrm{H}, 0,2.10765614,-2.1947903616,1.0118045047$ $\mathrm{H}, 0,4.6291827673,-2.4638248146,1.073305172$ $\mathrm{H}, 0,2.8262157851,-2.1626233556,-0.5880117777$ $\mathrm{H}, 0,4.0054096209,-1.0957057489,2.026432076$ $\mathrm{H}, 0,4.8049638478,1.2140987848,-0.9367337249$ C, $0,-1.4283899671,1.181910342,-0.3647297249$ $\mathrm{H}, 0,-1.4414824561,0.9444972895,-1.4399116483$ C, $0,-0.1335621602,-1.265278653,-0.0661326652$ $\mathrm{H}, 0,0.3183078567,-2.2542277077,-0.1064565621$ C, $0,-1.6415613946,-1.2704485423,-0.1528551463$ $\mathrm{H}, 0,-1.9270080431,-1.3974280161,-1.2079764384$ C,0,-2.2099040474,0.0607779763,0.3463577526 C, $0,-3.6575076029,0.3925849643,-0.055153979$ C, $0,-2.2757645299,2.4451874999,-0.1363664742$ $\mathrm{H}, 0,-1.9272347907,2.9795801003,0.7548269748$ $\mathrm{H}, 0,-2.203132723,3.1398149615,-0.9766981682$ $\mathrm{C}, 0,-3.7248560581,1.9260622586,0.0689208086$ $\mathrm{H}, 0,-4.4271071159,2.3301239184,-0.6668683269$ $\mathrm{H}, 0,-4.1045071538,2.1993388961,1.0572446415$ C, $0,-2.1052592333,0.1592400618,1.8749876506$ $\mathrm{H}, 0,-2.8408672399,-0.4960434373,2.3502787959$ $\mathrm{H}, 0,-1.1139766274,-0.1420365035,2.2249237017$ 
$\mathrm{H}, 0,-2.2881724674,1.1798492151,2.2247802113$

$0,0,6.1459116583,-0.7787142385,-0.0637585653$

$\mathrm{H}, 0,4.7422071345,1.5025014057,0.7904952181$

$0,0,-2.1192729203,-2.403449233,0.5757434676$

$\mathrm{H}, 0,-3.0114771064,-2.6054750432,0.2327329013$

$0,0,-4.5529472527,-2.6475342828,-0.8121644538$

$\mathrm{H}, 0,-4.376485982,-1.7744585554,-1.2185241589$

$\mathrm{H}, 0,-4.4266858169,-3.2963297855,-1.516467275$

$\mathrm{H}, 0,-4.3914211062,-0.1142248916,0.5843338751$

O,0,-3.8771715957,-0.0169631896,-1.4162906107

$\mathrm{H}, 0,-4.6793836332,0.4212955783,-1.7345241633$

diradical intermediate trans-5OH (T1) - water and hydroxyl complexed at carbonyl

Charge $=0$ Multiplicity $=3$

C, $0,-1.1039243831,2.2986552116,-0.1877695822$

C, $0,-1.9009261122,1.0410239703,-0.4002270268$

C, $0,-1.3206429451,-0.2002488603,-0.4680179024$

C, $0,0.1142231892,-0.352744157,-0.3743284887$

C, $0,0.977778308,0.8982830047,-0.3736753098$

C, $0,0.2834646189,2.0308672257,0.3831382358$

$\mathrm{H}, 0,-1.0192896385,2.8248351771,-1.1511395408$

$\mathrm{H}, 0,-1.6674793254,2.9701995705,0.4718585777$

$\mathrm{H}, 0,1.1052251811,1.2310677274,-1.4189606198$

$\mathrm{H}, 0,0.1997490366,1.7482770067,1.4402978335$

$\mathrm{H}, 0,0.8929463895,2.9394081977,0.3328037966$

C, $0,-2.1843292025,-1.4294699951,-0.695787506$

$\mathrm{C}, 0,-3.388682525,1.2524053708,-0.4674541198$

C $, 0,-4.208234709,0.024394922,-0.7556392662$

C, $0,-3.4912856576,-1.1012064044,-1.4300239654$

$\mathrm{H}, 0,-1.6421576008,-2.1588364484,-1.3010307108$

$\mathrm{H}, 0,-4.1481098062,-1.9706419834,-1.498837195$

$\mathrm{H}, 0,-2.408369347,-1.919746078,0.2598841679$

$\mathrm{H}, 0,-3.2492665468,-0.7583165186,-2.4454874059$

$\mathrm{C}, 0,2.3432584472,0.5724081441,0.2134202237$

$\mathrm{H}, 0,2.1727630519,0.2889056261,1.2629692331$

C, $0,0.7251854953,-1.6331567454,-0.275937845$

$\mathrm{H}, 0,0.0839084408,-2.5072901902,-0.200943933$

C, $0,2.0845120619,-1.8313672657,-0.271172007$

C, $0,2.9918250035,-0.6547479236,-0.4547995849$

C, $0,4.3569581556,-0.6879980986,0.2454173543$

C, $0,3.4500984426,1.630772997,0.1896239296$

$\mathrm{H}, 0,3.4320333887,2.1836634368,-0.7569326971$

$\mathrm{H}, 0,3.340965589,2.3612779175,0.9951408223$

C, $0,4.7613708711,0.8038746623,0.3210305983$

$\mathrm{H}, 0,5.2761120205,0.9924724469,1.2680990825$

$\mathrm{H}, 0,5.464560805,1.0469622076,-0.4804373768$

C, $0,3.2381702775,-0.4517254008,-1.9648440811$

$\mathrm{H}, 0,3.741886701,-1.3331389161,-2.3751067037$

$\mathrm{H}, 0,2.2953293969,-0.3239228677,-2.5031302712$

$\mathrm{H}, 0,3.8700056453,0.4201733123,-2.1646132284$ 
0,0,-5.3961883116,-0.027711529,-0.4540492922 O,0,4.1824799598,-1.2527983331,1.5490639599 $\mathrm{H}, 0,2.4941980806,-2.8364248973,-0.2050165453$ $\mathrm{H}, 0,-3.7549594977,1.701793962,0.4658886762$ $\mathrm{H}, 0,-3.6254863183,1.9820122111,-1.2587814019$ $\mathrm{O}, 0,-1.6672333718,0.0303267659,2.2267542237$ $\mathrm{H}, 0,5.0883779137,-1.2895680928,-0.3088285683$ $\mathrm{H}, 0,5.0135369098,-1.1540289755,2.0331407658$ $\mathrm{H}, 0,-2.5837343074,-0.2858708267,2.0253160334$ $\mathrm{O}, 0,-4.2756187303,-0.9792821893,2.1077351709$ $\mathrm{H}, 0,-4.9815977367,-0.5274483424,1.6229814603$ $\mathrm{H}, 0,-4.5314233081,-0.934385035,3.0391770302$

hydration TS2 trans-5OH (T1)

Charge $=0$ Multiplicity $=3$

C,0,-1.0761339682,2.3131647782,-0.0177847071 C, $0,-1.9120158951,1.0774509617,-0.2112547741$ C, $0,-1.3313732025,-0.1554744537,-0.4815634789$ C, $0,0.134079797,-0.3378778022,-0.338071023$ C,0,0.9994326919,0.9076466996,-0.3167049411 C, $0,0.3264106548,2.018663092,0.495443255$ $\mathrm{H}, 0,-1.0220035979,2.821756997,-0.9914367088$ $\mathrm{H}, 0,-1.6070457656,2.9914216176,0.6584281864$ $\mathrm{H}, 0,1.1058432591,1.2748512315,-1.3528938538$ $\mathrm{H}, 0,0.2794638154,1.7108468075,1.5464879962$ $\mathrm{H}, 0,0.9360783071,2.9268636205,0.4449625103$ C,0,-2.1667713077,-1.3526483058,-0.8425418662 $\mathrm{C}, 0,-3.364848858,1.357071506,-0.5067865924$ $\mathrm{C}, 0,-4.2028908553,0.1168888759,-0.6389637415$ C, $0,-3.5624303784,-1.0143299161,-1.385994784$ $\mathrm{H}, 0,-1.6354548021,-1.9534402403,-1.5860210617$ $\mathrm{H}, 0,-4.2163872954,-1.8882385454,-1.3691877074$ $\mathrm{H}, 0,-2.2629329317,-1.9877724027,0.0489742283$ $\mathrm{H}, 0,-3.4647274671,-0.6710487506,-2.4246479158$ C,0,2.3720730391,0.5578510995,0.2413062122 $\mathrm{H}, 0,2.2170051251,0.280293056,1.2946580315$ C, $0,0.6882352174,-1.6047512426,-0.235177097$ $\mathrm{H}, 0,0.0277369754,-2.4667397078,-0.1873238455$ C, $0,2.0730340556,-1.8429026629,-0.2187542563$ C, $0,2.9868898551,-0.682330196,-0.4363219062$ C, $0,4.3725716508,-0.728814758,0.2206679975$ C, $0,3.4933888794,1.6004158644,0.1896149671$ $\mathrm{H}, 0,3.4531219836,2.1597418196,-0.7522516348$ $\mathrm{H}, 0,3.41770918,2.3267036869,1.0026351154$ C,0,4.7978651605,0.7573253229,0.2751151215 $\mathrm{H}, 0,5.3514224587,0.943169096,1.2005181157$ $\mathrm{H}, 0,5.4723271629,0.9881884933,-0.5541518433$ C, $0,3.1806921686,-0.4886426428,-1.9561543676$ $\mathrm{H}, 0,3.6789659718,-1.3673568669,-2.3780418318$ $\mathrm{H}, 0,2.2187322297,-0.3716402782,-2.4625957019$ 
$\mathrm{H}, 0,3.7958793338,0.3891804818,-2.1802755818$ $0,0,-5.339920115,0.0572879569,-0.1871173022$ $0,0,4.2286681315,-1.2833719775,1.5322423594$ $\mathrm{H}, 0,2.456282512,-2.8568299556,-0.1554390105$ $\mathrm{H}, 0,-3.7893413606,2.0119136866,0.2585330708$ $\mathrm{H}, 0,-3.4285001335,1.8909043811,-1.4682207252$ $\mathrm{O}, 0,-2.0489300353,0.5475713622,1.785722098$ $\mathrm{H}, 0,5.0778438262,-1.3435069881,-0.3524606396$ $\mathrm{H}, 0,5.0713562307,-1.1839141599,1.9958131292$ $\mathrm{H}, 0,-2.6862802387,-0.1924100728,1.7549308398$ $\mathrm{O}, 0,-4.0788745179,-1.6380089677,1.905424079$ $\mathrm{H}, 0,-4.8719501779,-1.2677516395,1.4914500881$ $\mathrm{H}, 0,-4.3008967692,-1.7295949616,2.8415484979$

diol trans-5OH (T1) - water complexed between $\mathrm{C}_{5} \mathrm{OH}$ and $\mathrm{C}_{3}$ carbonyl Charge $=0$ Multiplicity $=3$

C,0,-1.1343996401,2.234808356,-0.15971993

C, $0,-1.9966174468,0.9909565876,0.0753863986$

C,0,-1.3367480607,-0.2299182723,-0.5213000951

C, $0,0.1255729254,-0.3946931677,-0.277382703$

C, $0,0.9794074091,0.8532323536,-0.4110041434$

C, $0,0.3044131723,2.0374500672,0.3004179404$

$\mathrm{H}, 0,-1.1482600848,2.4595838646,-1.2328567543$

$\mathrm{H}, 0,-1.6009719092,3.0789607606,0.3614803903$

$\mathrm{H}, 0,1.052389015,1.113421339,-1.4822270949$

$\mathrm{H}, 0,0.3320864741,1.862482997,1.3833591165$

$\mathrm{H}, 0,0.8815725402,2.9482660615,0.1060509224$

C, $0,-2.1581124018,-1.4375574675,-0.8428187818$

C,0,-3.4050211659,1.2370446953,-0.5056390228

C,0,-4.2120347907,-0.0215958878,-0.6678594738

C, $0,-3.5376450365,-1.1149235505,-1.4452427858$

$\mathrm{H}, 0,-1.6081972278,-2.0933407052,-1.525622489$

$\mathrm{H}, 0,-4.176209692,-1.9999872029,-1.4703964968$

$\mathrm{H}, 0,-2.3116627339,-2.0268986441,0.0782750914$

$\mathrm{H}, 0,-3.4038185878,-0.7445054711,-2.469302367$

C, $0,2.369050658,0.5924989828,0.1555272175$

$\mathrm{H}, 0,2.2451077743,0.4832788191,1.2435415472$

C,0,0.6709312207,-1.6110405887,0.0495477553

$\mathrm{H}, 0,0.0071859498,-2.4580671236,0.2100861808$

C,0,2.077664358,-1.8502891267,0.1045792099

C, $0,2.9738204783,-0.7385494837,-0.3321144185$

C, $0,4.382636885,-0.670692834,0.2729305797$

C, $0,3.4796303745,1.6198396146,-0.0909513692$

$\mathrm{H}, 0,3.401666342,2.0309797629,-1.1040825621$

$\mathrm{H}, 0,3.4243046631,2.4601466871,0.6056650644$

C, $0,4.7951161861,0.8064220958,0.0725401163$

$\mathrm{H}, 0,5.3850079943,1.1380291737,0.9324364773$

$\mathrm{H}, 0,5.4319557569,0.9040105873,-0.8111586788$

C,0,3.1139734792,-0.7850324323,-1.8702154307

$\mathrm{H}, 0,3.608898338,-1.7151297047,-2.1678073049$ 
$\mathrm{H}, 0,2.1327957272,-0.7546473048,-2.3521891682$ $\mathrm{H}, 0,3.7082353364,0.0520584174,-2.2522292787$ O,0,-5.3401826576,-0.1402792488,-0.2005824206 $\mathrm{O}, 0,4.2954518776,-1.0075136948,1.6617130833$ $\mathrm{H}, 0,2.4599415254,-2.8520558201,0.2741626072$ $\mathrm{H}, 0,-3.9409112829,1.9552665038,0.1201817995$ $\mathrm{H}, 0,-3.2917187381,1.6591164425,-1.5127405867$ $\mathrm{O}, 0,-2.0872955019,0.8345583632,1.5070845017$ $\mathrm{H}, 0,5.0734795277,-1.3663510731,-0.2197829786$ $\mathrm{H}, 0,5.1613619418,-0.8527423691,2.0633139089$ $\mathrm{H}, 0,-2.67043651,0.0784367855,1.7221589653$ $\mathrm{O}, 0,-3.9054283809,-1.2232813329,2.1753589503$ $\mathrm{H}, 0,-4.7505458679,-0.9727702375,1.7744918901$ $\mathrm{H}, 0,-4.0559062132,-1.1976795746,3.1299426203$

diol trans-5OH (SO) - water complexed between $\mathrm{C}_{5} \mathrm{OH}$ and $\mathrm{C}_{3}$ carbonyl Charge $=0$ Multiplicity $=1$

C,0,-1.1059609353,2.1727429745,-0.2248436575 C,0,-1.9689069066,0.9506613859,0.0783548855 C,0,-1.2235589253,-0.3717615506,-0.1105798705 C, $0,0.1051411134,-0.4262024799,-0.3654524572$ C,0,0.9692185716,0.8373475947,-0.4227405383 C, $0,0.3062104247,1.9969753331,0.3109656949$ $\mathrm{H}, 0,-1.0557684319,2.3129862323,-1.3111832521$ $\mathrm{H}, 0,-1.605485212,3.0501262845,0.198648414$ $\mathrm{H}, 0,1.0810144264,1.1354261102,-1.4771126755$ $\mathrm{H}, 0,0.2858556248,1.7916068125,1.3900263184$ $\mathrm{H}, 0,0.8884273112,2.912225473,0.1617307956$ $\mathrm{C}, 0,-2.1131083485,-1.5777401546,0.0917973258$ C,0,-3.2218303591,0.9917459221,-0.821831671 C, $0,-4.0812413831,-0.2412387451,-0.7363048553$ C,0,-3.3563436057,-1.5551383474,-0.8170713446 $\mathrm{H}, 0,-1.5862135042,-2.5161166333,-0.0687393834$ $\mathrm{H}, 0,-4.0474302062,-2.3651505255,-0.573103578$ $\mathrm{H}, 0,-2.4497858172,-1.5908119199,1.1366548311$ $\mathrm{H}, 0,-3.0378627679,-1.6718360268,-1.8620209974$ C,0,2.3453036664,0.4940939907,0.1313206049 $\mathrm{H}, 0,2.1851203208,0.0831836769,1.1397789264$ C, $0,0.8143907713,-1.7108971557,-0.5630320972$ $\mathrm{H}, 0,0.2203680074,-2.6163155427,-0.6263418796$ C,0,2.1484408144,-1.8260959243,-0.6908123509 C, $0,3.0359159717,-0.6182826564,-0.6745249968$ C, $0,4.3770997899,-0.72660276,0.0663113802$ C, $0,3.4237547866,1.5728886107,0.2484734723$ $\mathrm{H}, 0,3.4373943228,2.2061867249,-0.6462999554$ $\mathrm{H}, 0,3.2657134544,2.2273942121,1.1093715608$ C, $0,4.7399876626,0.7507153117,0.36665275$ $\mathrm{H}, 0,5.1729925317,0.8164653826,1.3694629414$ $\mathrm{H}, 0,5.4978390296,1.1086004672,-0.3356168538$ C, $0,3.345371819,-0.2249645895,-2.1337731244$ 
$\mathrm{H}, 0,3.8327873903,-1.0651507343,-2.6396303779$ $\mathrm{H}, 0,2.4324083129,0.011753425,-2.685402053$ $\mathrm{H}, 0,4.0194823695,0.6356050453,-2.1932523407$ O,0,-5.3013579805,-0.1708207015,-0.636248029 $0,0,4.1742417575,-1.4797096603,1.2661050275$ $\mathrm{H}, 0,2.5919796349,-2.8049276223,-0.8693865321$ $\mathrm{H}, 0,-3.8184847094,1.8799954558,-0.5935897852$ $\mathrm{H}, 0,-2.8886678284,1.0527960747,-1.8667413672$ $\mathrm{O}, 0,-2.3653298415,1.0801205169,1.4553179813$ $\mathrm{H}, 0,5.1414064824,-1.2263740887,-0.5415299873$ $\mathrm{H}, 0,4.9964680388,-1.4625992685,1.7743093117$ $\mathrm{H}, 0,-3.0807438966,0.4537882966,1.6812068361$ $0,0,-4.5672747597,-0.5357666014,2.2273506485$ $\mathrm{H}, 0,-5.3142855186,-0.3506610229,1.6401512387$ $\mathrm{H}, 0,-4.8431594696,-0.2189596024,3.0981810663$

\section{$17 \beta$}

trenbolone (SO) - complex with two waters near carbonyl Charge $=0$ Multiplicity $=1$

C, $0,-0.7828647999,2.4394142514,-0.0116878066$

$\mathrm{C}, 0,-1.6793991344,1.261146792,-0.2867257321$

C, $0,-1.0837857846,-0.0628318803,-0.486584208$

C, $0,0.2734688251,-0.2238264353,-0.4573560054$

C, $0,1.2081678722,0.9642422288,-0.2267534933$

C, $0,0.5295342845,2.0245270224,0.6335001476$

$\mathrm{H}, 0,-0.5659045386,2.9162008206,-0.9785705786$

$\mathrm{H}, 0,-1.3248023483,3.1745971888,0.5897826878$

$\mathrm{H}, 0,1.4319301343,1.4199142546,-1.2045039645$

$\mathrm{H}, 0,0.3482679882,1.6161972223,1.6368927293$

$\mathrm{H}, 0,1.1860568276,2.8934195365,0.7421252738$

C, $0,-2.063410318,-1.2123042684,-0.6203584819$

C, $0,-3.02781461,1.4572425989,-0.3400607102$

C, $0,-3.9487754461,0.4268635324,-0.7851808741$

C, $0,-3.335471445,-0.8151001725,-1.3688147924$

$\mathrm{H}, 0,-1.6173783544,-2.0642451587,-1.131378999$

$\mathrm{H}, 0,-4.0710577568,-1.6226531965,-1.3653301385$

$\mathrm{H}, 0,-2.3420379815,-1.5514028604,0.3878121421$

$\mathrm{H}, 0,-3.0972360224,-0.5816980826,-2.415830729$

$\mathrm{H}, 0,-3.0449758693,0.1864819127,1.925898441$

$\mathrm{C}, 0,2.5073409092,0.4676073514,0.3883006505$

$\mathrm{H}, 0,2.2409865445,-0.0183053546,1.3409872954$

C, $0,0.8920013977,-1.5490779025,-0.6164875269$

$\mathrm{H}, 0,0.2409294925,-2.4059579909,-0.7480825633$

C, $0,2.223353353,-1.7576169916,-0.6152950994$

$\mathrm{H}, 0,2.613353993,-2.7628061458,-0.7712611052$

C, $0,3.1825497531,-0.6177547391,-0.4622654083$

C, $0,4.4301953315,-0.8726959334,0.3947597219$

$\mathrm{H}, 0,4.1319340625,-1.4444566685,1.2837607872$

C, $0,3.6367427628,1.4602744977,0.6860510776$ 
$\mathrm{H}, 0,3.7470403839,2.1761339759,-0.1366788754$ $\mathrm{H}, 0,3.4497227768,2.0339225504,1.5970968291$ C,0,4.890342902,0.5488678915,0.805710183 $\mathrm{H}, 0,5.3043124396,0.5467630451,1.8171534071$ $\mathrm{H}, 0,5.6848094905,0.8724105836,0.1258670587$ C, $0,3.6005744041,-0.1340075037,-1.864490963$ $\mathrm{H}, 0,4.0723475211,-0.9531856296,-2.4144541953$ $\mathrm{H}, 0,2.7301502781,0.194739364,-2.4383268579$ $\mathrm{H}, 0,4.3161629606,0.6928550894,-1.8140857504$ $\mathrm{O}, 0,5.4114338506,-1.614090733,-0.3216022601$ $\mathrm{H}, 0,6.1320756417,-1.8284536823,0.2859202756$ O,0,-5.1785099395,0.5824011344,-0.7336206135 $\mathrm{H}, 0,-3.4515320327,2.427558044,-0.0928013476$ $\mathrm{O}, 0,-3.6340985017,-0.2656732067,2.5454373452$ $\mathrm{H}, 0,-4.2509212616,-0.7756140879,1.9844633477$ $\mathrm{O}, 0,-5.4944257339,-1.7241654608,0.9739921769$ $\mathrm{H}, 0,-6.3049480187,-1.8790126293,1.4766500309$ $\mathrm{H}, 0,-5.7226895732,-1.0508793041,0.3111204617$

trenbolone (T1) - complex with two waters near carbonyl Charge $=0$ Multiplicity $=3$

C,0,-0.7643133573,2.4449720437,0.0455202937

$\mathrm{C}, 0,-1.6473803112,1.2603166592,-0.2726161306$

$\mathrm{C}, 0,-1.1272855721,-0.0084239642,-0.4586904978$

C, $0,0.3292817506,-0.2544807037,-0.3595983058$

C, $0,1.2378483559,0.9545172716,-0.2218338101$

C, $0,0.5676633274,2.0203158395,0.6487682911$

$\mathrm{H}, 0,-0.5849380707,3.0120661515,-0.8779862042$

$\mathrm{H}, 0,-1.3019032849,3.1169242245,0.7225023679$

$\mathrm{H}, 0,1.3908744341,1.3973561722,-1.2213731096$

$\mathrm{H}, 0,0.4083128736,1.6103116586,1.6553392159$

$\mathrm{H}, 0,1.2296737617,2.8867432897,0.7473694199$

C, $0,-2.056900255,-1.1701248425,-0.6942681334$

C,0,-3.0542793025, 1.4734678457,-0.3075703599

C, $0,-3.9775264792,0.474589948,-0.7441578781$

C,0,-3.3845296248,-0.7733007995,-1.3388623885

$\mathrm{H}, 0,-1.5755648212,-1.9211077278,-1.3232049501$

$\mathrm{H}, 0,-4.1105335108,-1.5880615787,-1.2863755223$

$\mathrm{H}, 0,-2.2436573266,-1.6539795932,0.2771696376$

$\mathrm{H}, 0,-3.2162827331,-0.5502955097,-2.4022107488$

$\mathrm{H}, 0,-3.0644712544,-0.1210242445,1.9329688754$

C, $0,2.5799736611,0.5156512614,0.3449928577$

$\mathrm{H}, 0,2.3824404906,0.1496340631,1.3658806135$

C,0,0.8456456044,-1.5402722556,-0.3525871969

$\mathrm{H}, 0,0.1668990279,-2.3878327432,-0.3783044465$

C, $0,2.2257775885,-1.8189266778,-0.3319090917$

$\mathrm{H}, 0,2.579276836,-2.8459060531,-0.346243487$

C,0,3.1820599594,-0.6767455003,-0.4198924573

C, $0,4.5118180305,-0.8228082672,0.3301374997$

$\mathrm{H}, 0,4.3054946468,-1.2721699245,1.3110371269$ 
C, $0,3.7318077869,1.5260068709,0.430255239$

$\mathrm{H}, 0,3.7523450166,2.1600007604,-0.4635335913$

$\mathrm{H}, 0,3.6344509384,2.1853900734,1.296132167$

$\mathrm{C}, 0,5.0022691626,0.6341807182,0.506674111$

$\mathrm{H}, 0,5.5385159064,0.7545074306,1.4512803886$

$\mathrm{H}, 0,5.7021431737,0.870545093,-0.3011760134$

C, $0,3.4403580825,-0.3604385894,-1.9086862164$

$\mathrm{H}, 0,3.8991835981,-1.2231855195,-2.3993417371$

$\mathrm{H}, 0,2.5016737408,-0.1415251534,-2.4251825428$

$\mathrm{H}, 0,4.1118341989,0.4958068333,-2.0318889094$

$\mathrm{O}, 0,5.420379444,-1.6598436768,-0.3773805068$

$\mathrm{H}, 0,6.192038958,-1.8128937454,0.1842652274$

$0,0,-5.219145445,0.6437564586,-0.6844635031$

$\mathrm{H}, 0,-3.4593114102,2.431184177,0.0096531511$

$\mathrm{O}, 0,-3.6985327338,-0.5695277738,2.508243557$

$\mathrm{H}, 0,-4.372692528,-0.9471970608,1.9099026534$

$\mathrm{O}, 0,-5.7701956671,-1.6537554535,0.8934516995$

$\mathrm{H}, 0,-6.5796818916,-1.6180127594,1.4199649958$

$\mathrm{H}, 0,-5.8416951657,-0.9201706169,0.25581245$

hydration TS1 (common) (T1)

Charge $=0$ Multiplicity $=3$

C, $0,-0.864002301,2.2933736016,-0.3405696027$

C,0,-1.6756466962,1.027447569,-0.3390206301

C, $0,-1.1279449001,-0.2150110141,-0.1986089434$

C, $0,0.3099024795,-0.3906467493,-0.1180158028$

C, $0,1.183940428,0.8315288068,-0.3533861504$

C, $0,0.5265505673,2.0781797153,0.2441599194$

$\mathrm{H}, 0,-0.7883485764,2.6703813503,-1.371581346$

$\mathrm{H}, 0,-1.4040329141,3.0640161258,0.2230274712$

$\mathrm{H}, 0,1.2717051203,0.9955457507,-1.4416738181$

$\mathrm{H}, 0,0.4518272495,1.9485481014,1.3324145646$

$\mathrm{H}, 0,1.155641469,2.9553603941,0.060218875$

C,0,-2.0692092347,-1.4031677774,-0.1464550366

C,0,-3.1601270418,1.1929803146,-0.4161924877

C,0,-3.9404574009,0.0494595639,-0.9981583798

C, $0,-3.2127565328,-1.2526243463,-1.1542812865$

$\mathrm{H}, 0,-1.5457978012,-2.3333254334,-0.3678992357$

$\mathrm{H}, 0,-3.9325426378,-2.0728097523,-1.0942057037$

$\mathrm{H}, 0,-2.4852408574,-1.5030392334,0.8654399662$

$\mathrm{H}, 0,-2.7976565442,-1.2458628707,-2.1723528045$

$\mathrm{H}, 0,-3.5542576552,1.2514250311,0.6837761108$

C, $0,2.569218992,0.5867466517,0.2260246811$

$\mathrm{H}, 0,2.4390651744,0.4898933526,1.3162928208$

C, $0,0.9051919336,-1.6370857512,0.1906666978$

$\mathrm{H}, 0,0.2601675289,-2.473154494,0.4458041999$

C, $0,2.2665900791,-1.8542470877,0.1816095404$

$\mathrm{H}, 0,2.6694409359,-2.8385438671,0.4087448804$

C, $0,3.17934237,-0.7460302963,-0.2416715717$

C, $0,4.5430144775,-0.6613234057,0.4557079553$ 
$\mathrm{H}, 0,4.3948345842,-0.851611218,1.5275398337$ C, $0,3.6830805596,1.6139246988,-0.0139461736$ $\mathrm{H}, 0,3.6436168525,1.9890803611,-1.0431589525$ $\mathrm{H}, 0,3.5957929715,2.4762103196,0.6517058771$ C,0,4.9866979636,0.805056664,0.2337291026 $\mathrm{H}, 0,5.5490332543,1.1778342155,1.0936473018$ $\mathrm{H}, 0,5.6512037617,0.8449421329,-0.6353472139$ C,0,3.3695071218,-0.8135739233,-1.7706885822 $\mathrm{H}, 0,3.8123790982,-1.7737256126,-2.0504526603$ $\mathrm{H}, 0,2.4068271482,-0.7302474633,-2.2826862821$ $\mathrm{H}, 0,4.026644927,-0.0182280694,-2.1380902728$ $\mathrm{O}, 0,5.4556502143,-1.6253456368,-0.0598676228$ $\mathrm{H}, 0,6.2698121755,-1.5774227613,0.4589786212$ $\mathrm{O}, 0,-5.1202697534,0.1865347496,-1.302932618$ $\mathrm{H}, 0,-3.481358546,2.145253645,-0.849086196$ $\mathrm{O}, 0,-4.005826988,0.9850276541,2.0629491653$ $\mathrm{H}, 0,-4.5135608468,0.158655968,1.8571761181$ $\mathrm{O}, 0,-5.4051431441,-1.3190915201,1.2998176816$ $\mathrm{H}, 0,-6.1073106742,-1.5588450614,1.9197235609$ $\mathrm{H}, 0,-5.8553900411,-1.1171574925,0.4660739786$

diradical intermediate (common) (T1)

Charge $=0$ Multiplicity $=3$

C,0,-1.192381888,2.2002911826,-0.6068256031 C, $0,-1.9520322633,0.9023793056,-0.5978845946$ C, $0,-1.3467152282,-0.3096053847,-0.3816187479$ C, $0,0.0839518046,-0.3988173231,-0.1985015639$ C, $0,0.921607258,0.8495473968,-0.4226022149$ C,0,0.1732612633,2.0923578175,0.0609671437 $\mathrm{H}, 0,-1.0766750469,2.5288772902,-1.6511869857$ $\mathrm{H}, 0,-1.7998800359,2.9709863984,-0.1159974514$ $\mathrm{H}, 0,1.0865639299,0.9666140897,-1.5081152231$ $\mathrm{H}, 0,0.0484180314,2.0268519868,1.1494183614$ $\mathrm{H}, 0,0.7666310444,2.9886542958,-0.1482975492$ C,0,-2.1789688706,-1.5810972947,-0.3917644081 C, $0,-3.4367874837,1.0496865058,-0.7878414842$ C, $0,-4.2118470667,-0.2366202493,-0.8757077746$ C, $0,-3.4309442352,-1.4489624985,-1.2703150234$ $\mathrm{H}, 0,-1.5887078478,-2.4122361049,-0.7823906455$ $\mathrm{H}, 0,-4.0656551314,-2.3351291567,-1.2100388553$ $\mathrm{H}, 0,-2.4700569592,-1.8541780565,0.630622676$ $\mathrm{H}, 0,-3.1173136712,-1.305206544,-2.3131666265$ $\mathrm{H}, 0,-3.8691817221,1.6620354491,0.0155096058$ C,0,2.2675765713,0.6885940902,0.2673810551 $\mathrm{H}, 0,2.0587514586,0.6179455032,1.3474439952$ C, $0,0.7155675781,-1.612603324,0.1897606436$ $\mathrm{H}, 0,0.0888619749,-2.4676869896,0.4287444876$ C,0,2.0772658538,-1.765437897,0.2816299149 $\mathrm{H}, 0,2.507153866,-2.7225618759,0.5686988449$ C,0,2.9700553379,-0.6279169785,-0.1087814085 
C, $0,4.2708029309,-0.458313113,0.6863059953$

$\mathrm{H}, 0,4.0492390037,-0.6212441271,1.7499664232$

C, $0,3.3498747304,1.7591438749,0.0778752413$

$\mathrm{H}, 0,3.3714917181,2.1032421197,-0.9626634458$

$\mathrm{H}, 0,3.1744108791,2.6348521695,0.7077094195$

$\mathrm{C}, 0,4.665492658,1.0191040266,0.4468118616$

$\mathrm{H}, 0,5.1476412643,1.4435705881,1.3311490093$

$\mathrm{H}, 0,5.3892229778,1.0615402887,-0.3735114161$

C,0,3.2812471933,-0.7329122023,-1.6154317962

$\mathrm{H}, 0,3.7945986526,-1.675503012,-1.8260648084$

$\mathrm{H}, 0,2.3580366572,-0.7200360846,-2.2013728894$

$\mathrm{H}, 0,3.92193103,0.0853083945,-1.9610637644$

$\mathrm{O}, 0,5.2621670123,-1.3945138108,0.2752839801$

$\mathrm{H}, 0,6.0338691388,-1.2880143862,0.8477334904$

$0,0,-5.4138029161,-0.2676398252,-0.6322675002$

$\mathrm{H}, 0,-3.638616434,1.602189142,-1.7198795809$

$0,0,-1.8145433504,0.4485302428,2.2030633447$

$\mathrm{H}, 0,-2.7370299055,0.1363641676,2.0253477475$

$0,0,-4.4716778495,-0.4489677064,2.1763291112$

$\mathrm{H}, 0,-4.8102592199,-0.1141285091,3.0178104996$

$\mathrm{H}, 0,-5.123443161,-0.1817273396,1.5116372276$

diradical intermediate cis- $120 \mathrm{H}(\mathrm{T} 1)$ - complexed water and hydroxyl radical at $\mathrm{C}_{17}$

Charge $=0$ Multiplicity $=3$

C, $0,-2.3295499952,-2.2489728768,0.3457233443$

C,0,-2.8502626509,-0.8665504522,0.0572702134

C, $0,-2.0238088949,0.181144938,-0.2552347467$

C, $0,-0.595503436,0.0084751177,-0.2928923425$

C, $0,-0.0097564051,-1.3124637357,0.1850451131$

C, $0,-0.9275432493,-2.4695329438,-0.2096075269$

$\mathrm{H}, 0,-2.3295140531,-2.407220477,1.4353062405$

$\mathrm{H}, 0,-3.0284925389,-2.9874635389,-0.0650568804$

$\mathrm{H}, 0,0.0337140388,-1.2952582877,1.2877987337$

$\mathrm{H}, 0,-0.9689222471,-2.5327342713,-1.3053601007$

$\mathrm{H}, 0,-0.5173200581,-3.4156967737,0.1593344231$

C,0,-2.6147489234,1.5473070995,-0.5606275644

C, $0,-4.3500710139,-0.7449118857,0.1010288022$

C, $0,-4.8992497661,0.6321295145,-0.1597677324$

C,0,-3.9683371123,1.76904995,0.1263454442

$\mathrm{H}, 0,-1.93745264,2.3331012206,-0.2186935516$

$\mathrm{H}, 0,-4.4281070251,2.7118412508,-0.176720639$

$\mathrm{H}, 0,-2.7252769339,1.6783318081,-1.6444476943$

$\mathrm{H}, 0,-3.8144275821,1.7837544331,1.2141130032$

$\mathrm{H}, 0,-4.8154602262,-1.4469725516,-0.6021170912$

C,0,1.4029445892,-1.4797837619,-0.3564457779

$\mathrm{H}, 0,1.3182180083,-1.5605289612,-1.452245389$

C, $0,0.2702450165,1.0209308403,-0.8080504708$

$\mathrm{H}, 0,-0.1764758801,1.9006799772,-1.2640675108$

C, $0,1.6376423571,0.9269949685,-0.7858255691$

$\mathrm{H}, 0,2.2537769208,1.7131305611,-1.2167081763$ 
C, $0,2.2710365922,-0.2372611549,-0.0884336897$

C, $0,3.6107276935,-0.7238309773,-0.6459947082$

C, $0,2.2664319568,-2.6524718067,0.1297153073$

$\mathrm{H}, 0,2.1066811975,-2.8292743846,1.1995390376$

$\mathrm{H}, 0,2.0258191825,-3.5813114555,-0.3934191738$

C, $0,3.7236165003,-2.1819259746,-0.1428216664$

$\mathrm{H}, 0,4.233023364,-2.8118751471,-0.8762275646$

$\mathrm{H}, 0,4.3223677858,-2.1942083544,0.7734651943$

C, $0,2.4037075983,0.066286912,1.4190581812$

$\mathrm{H}, 0,3.0702589105,0.9173462264,1.5812084837$

$\mathrm{H}, 0,1.4307493938,0.3244033409,1.845655$

$\mathrm{H}, 0,2.8082659952,-0.7886131512,1.9712792331$

O,0,-6.0462922017,0.7933699994,-0.5585318345

$\mathrm{H}, 0,-4.7144142593,-1.0420468841,1.0982858131$

$0,0,1.5225302569,3.318442959,0.955220926$

$\mathrm{H}, 0,2.4562278536,3.0865758548,0.6974761848$

$\mathrm{O}, 0,4.1716509341,2.8564502948,0.2426793353$

$\mathrm{H}, 0,4.3504622723,1.9163930245,0.034709481$

$\mathrm{H}, 0,4.2935505037,3.3285833848,-0.5922424081$

$\mathrm{H}, 0,3.5633580925,-0.7012518189,-1.742357616$

$0,0,4.6822903759,0.120605035,-0.2191579661$

$\mathrm{H}, 0,5.4706447026,-0.0851040843,-0.7400061038$

hydration TS2 cis- $12 \mathrm{OH}$ (T1)

Charge $=0$ Multiplicity $=3$

C, $0,-2.3619028015,-2.2325913434,0.3904737936$

C, $0,-2.9138537071,-0.8803296086,0.0660249145$

C, $0,-2.0630684127,0.2009767762,-0.3118613498$

C, $0,-0.689145709,0.0598999427,-0.2727203379$

C, $0,-0.0679594036,-1.2395919245,0.226782744$

C, $0,-0.9505473653,-2.4261453448,-0.1596949061$

$\mathrm{H}, 0,-2.350866243,-2.3602793689,1.4853844424$

$\mathrm{H}, 0,-3.042571103,-3.0028285415,0.006993534$

$\mathrm{H}, 0,-0.0167131782,-1.214959018,1.3276551146$

$\mathrm{H}, 0,-0.984989595,-2.5046818463,-1.2545917818$

$\mathrm{H}, 0,-0.5218396958,-3.3568046259,0.2256457422$

C, $0,-2.7615409301,1.4774600724,-0.73272372$

C, $0,-4.4024020384,-0.7328715308,0.0549600133$

C, $0,-4.9276879397,0.6708446419,0.2540171259$

C, $0,-3.9292693794,1.7914117098,0.2097214509$

$\mathrm{H}, 0,-2.0794967074,2.3270381281,-0.7310071123$

$\mathrm{H}, 0,-4.442140375,2.716130301,-0.0643997608$

$\mathrm{H}, 0,-3.1454631943,1.3663922648,-1.7550318486$

$\mathrm{H}, 0,-3.5497359119,1.9083129124,1.2348292132$

$\mathrm{H}, 0,-4.8094000897,-1.0755039222,-0.9128361747$

C, $0,1.3456892959,-1.3477390093,-0.3224164805$

$\mathrm{H}, 0,1.2645556661,-1.3390380923,-1.4218033233$

C, $0,0.2109574482,1.0859653349,-0.8134454362$

$\mathrm{H}, 0,-0.212277618,1.889989013,-1.406178091$

C,0,1.5819289719,1.0928291622,-0.6007996101 
$\mathrm{H}, 0,2.1983500988,1.7043752791,-1.2565385208$ C, $0,2.2113988243,-0.1203418374,0.0549310755$ C,0,3.5457287241,-0.5675563777,-0.5605215983 C,0,2.2099189435,-2.5552093766,0.0595353546 $\mathrm{H}, 0,2.0571382185,-2.8232755372,1.1109003129$ $\mathrm{H}, 0,1.9621552591,-3.434410344,-0.5401873933$ C,0,3.6613573626,-2.0622961743,-0.1858890349 $\mathrm{H}, 0,4.1643615386,-2.6245799596,-0.9764338595$ $\mathrm{H}, 0,4.2700621019,-2.1509832454,0.7194182418$ C,0,2.3594850379,0.0400193281,1.5755140122 $\mathrm{H}, 0,3.0729811531,0.8348880093,1.8048305351$ $\mathrm{H}, 0,1.4065590425,0.3098185224,2.035856383$ $\mathrm{H}, 0,2.7189573494,-0.8857000843,2.0352935241$ $\mathrm{O}, 0,-6.1209815715,0.8650494603,0.4447247453$ $\mathrm{H}, 0,-4.8679838898,-1.3809407728,0.8074095698$ $0,0,1.4998398602,2.6864933467,0.6807890561$ $\mathrm{H}, 0,2.4505292478,2.8613555579,0.5378262769$ $\mathrm{O}, 0,4.351039682,2.9657605017,0.1278829545$ $\mathrm{H}, 0,4.4534693271,2.0003693164,-0.0047720566$ $\mathrm{H}, 0,4.351614142,3.3468657563,-0.7598186391$ $\mathrm{H}, 0,3.4820264838,-0.4516495745,-1.6505498502$ $\mathrm{O}, 0,4.6385979989,0.2169135754,-0.082197628$ $\mathrm{H}, 0,5.4327675748,-0.0253854597,-0.5789651976$

diol cis-12OH (T1) - water complexed between C12 and C17 Charge $=0$ Multiplicity $=3$

C,0,-2.3592348003,-2.2517381943,0.3094889314 $\mathrm{C}, 0,-2.8856495713,-0.9095146617,-0.104621428$ $\mathrm{C}, 0,-2.0438471867,0.2101908565,-0.0932520518$ C, $0,-0.7143541782,0.1030412946,0.3104851855$ $\mathrm{C}, 0,-0.0497924821,-1.2453955265,0.544021694$ $\mathrm{C}, 0,-0.8787505103,-2.394145876,-0.0357693853$ $\mathrm{H}, 0,-2.5029996021,-2.3802922339,1.3948769515$ $\mathrm{H}, 0,-2.9434347242,-3.0446778157,-0.1717911664$ $\mathrm{H}, 0,0.0760723942,-1.4093002933,1.6276040307$ $\mathrm{H}, 0,-0.769516964,-2.3867664189,-1.1291592819$ $\mathrm{H}, 0,-0.4908454048,-3.3530288988,0.3233184784$ C, $0,-2.6180085279,1.5597735641,-0.4820616645$ C, $0,-4.3409959018,-0.8319390688,-0.4605194628$ C, $0,-4.8832034042,0.5563780903,-0.694020675$

C,0,-4.0979739997,1.6876796523,-0.0991528046 $\mathrm{H}, 0,-2.0529291564,2.363246263,-0.0029951385$ $\mathrm{H}, 0,-4.5255079167,2.6393211783,-0.4215365956$ $\mathrm{H}, 0,-2.5112714283,1.7051148937,-1.5645389682$ $\mathrm{H}, 0,-4.1944833394,1.6077789035,0.9923674611$ $\mathrm{H}, 0,-4.574234938,-1.4483801027,-1.3397043199$ C,0,1.3373561095,-1.1951378301,-0.106401653 $\mathrm{H}, 0,1.1620619264,-1.0341308944,-1.1839234353$ $\mathrm{C}, 0,0.1513935208,1.282770001,0.5403178412$ $\mathrm{H}, 0,0.0443160896,1.8379323339,1.4736541953$ 
C, $0,1.5223366893,1.2899352099,-0.0623147036$ $\mathrm{H}, 0,1.4518419442,1.2760259234,-1.1643102664$ C, $0,2.2307767478,-0.0244666639,0.3598197188$ C, $0,3.5107477103,-0.3724686702,-0.4194734713$ C, $0,2.2352863281,-2.434176856,0.0285990448$ $\mathrm{H}, 0,2.1687638517,-2.8410549521,1.0440797597$ $\mathrm{H}, 0,1.9380109887,-3.228000469,-0.6611114928$ C,0,3.6606040669,-1.9004001401,-0.2613711118 $\mathrm{H}, 0,4.1011913157,-2.3434791463,-1.1580463884$ $\mathrm{H}, 0,4.3386292963,-2.1041894566,0.5740828822$ C,0,2.4997664002,-0.0394741666, 1.8680630879 $\mathrm{H}, 0,3.1679949785,0.7792944527,2.1466329827$ $\mathrm{H}, 0,1.5751052739,0.0754216323,2.4402324951$ $\mathrm{H}, 0,2.9729511293,-0.975514612,2.1803330487$ $\mathrm{O}, 0,-5.9207755411,0.7295007341,-1.3218505624$ $\mathrm{H}, 0,-4.94458683,-1.2585945948,0.3584398176$ $\mathrm{O}, 0,2.1965184237,2.4693470273,0.3565410183$ $\mathrm{H}, 0,2.9416634046,2.6277747033,-0.2541514097$ $0,0,4.3635528687,2.6726551605,-1.4199396906$ $\mathrm{H}, 0,4.6428663363,1.8456885309,-0.975272143$ $\mathrm{H}, 0,4.0505989471,2.4018306614,-2.2934146651$ $\mathrm{H}, 0,3.3493022035,-0.117104493,-1.4778129437$ $\mathrm{O}, 0,4.6526466259,0.3514139326,0.0499305969$ $\mathrm{H}, 0,5.4497548363,-0.112687964,-0.2435383425$

diol cis- $12 \mathrm{OH}(\mathrm{SO})$ - water complexed between $\mathrm{C}_{12}$ and $\mathrm{C}_{17}$ Charge $=0$ Multiplicity $=1$

C,0,2.3082555837,2.2576743531,-0.2027060077 C, $0,2.8936433031,0.8845765617,0.0123780978$ C,0,2.1433747022,-0.2348558293,0.0929718469 $\mathrm{C}, 0,0.6668422739,-0.1642704148,-0.0223286349$ $\mathrm{C}, 0,0.0398599502,1.2181982582,0.0492771664$ $\mathrm{C}, 0,0.8858954913,2.2125456869,-0.745921107$ $\mathrm{H}, 0,2.3294732791,2.798744311,0.7549387412$ $\mathrm{H}, 0,2.9612260115,2.8205312323,-0.8800974615$ $\mathrm{H}, 0,0.0531715207,1.5426375257,1.1035191821$ $\mathrm{H}, 0,0.9003613235,1.902654078,-1.7996507692$ $\mathrm{H}, 0,0.4326759269,3.2088010827,-0.703801901$ C, $0,2.7833851707,-1.5921497636,0.3024465588$ C,0,4.3999393682,0.8693111826,0.1005509678 C, $0,5.0209066499,-0.4832946896,0.3234751471$ C, $0,4.1491327548,-1.4996032687,0.9933891438$ $\mathrm{H}, 0,2.1346033315,-2.2155062715,0.9232698449$ $\mathrm{H}, 0,4.6591253565,-2.4645118469,1.022188971$ $\mathrm{H}, 0,2.8865515804,-2.1127454111,-0.6582637006$ $\mathrm{H}, 0,3.9995585368,-1.1492543333,2.0239537718$ $\mathrm{H}, 0,4.8421501211,1.3133073218,-0.7991978131$ $\mathrm{C}, 0,-1.4005206472,1.1664692697,-0.434155988$ $\mathrm{H}, 0,-1.3719439783,0.9526995939,-1.515754044$ C,0,-0.0890455877,-1.2663768109,-0.1850426705 
$\mathrm{H}, 0,0.3750112063,-2.2481888598,-0.2513100958$ C, $0,-1.5953530988,-1.2870320435,-0.3048592142$ $\mathrm{H}, 0,-1.8643800598,-1.3831892177,-1.3705129699$ $\mathrm{C}, 0,-2.2030499514,0.0219639756,0.2178343499$ C,0,-3.6063671009,0.3647296241,-0.3086617916 C, $0,-2.2618258214,2.4231165853,-0.2216313931$ $\mathrm{H}, 0,-1.9883260504,2.9130388696,0.7197509326$ $\mathrm{H}, 0,-2.117285883,3.1536236723,-1.0211601502$ C,0,-3.7164454056,1.8941543158,-0.162451117 $\mathrm{H}, 0,-4.3542816394,2.3185263171,-0.9418795555$ $\mathrm{H}, 0,-4.1799800514,2.121245955,0.8034414617$ C,0,-2.1659198255,0.0797104795,1.7479577026 $\mathrm{H}, 0,-2.8599720868,-0.6451352319,2.1794598469$ $\mathrm{H}, 0,-1.1658921044,-0.1514198848,2.1260431383$ $\mathrm{H}, 0,-2.4464846519,1.0720761208,2.1151436079$ $\mathrm{O}, 0,6.1791334054,-0.7122831298,-0.0034732223$ $\mathrm{H}, 0,4.7239392244,1.5065447105,0.9387074017$ $\mathrm{O}, 0,-2.0540000265,-2.4556121474,0.3766246596$ $\mathrm{H}, 0,-2.9285696362,-2.6897639601,0.0107095264$ $0,0,-4.5760354039,-2.8172483247,-0.8120055482$ $\mathrm{H}, 0,-4.7636407535,-1.9353112146,-0.4268071598$ $\mathrm{H}, 0,-4.442097174,-2.6623823852,-1.7565185333$ $\mathrm{H}, 0,-3.6514152097,0.0846100367,-1.3718873151$ $0,0,-4.6409603691,-0.3373332564,0.3880606065$ $\mathrm{H}, 0,-5.4871877453,0.0852297256,0.1820489096$

diradical intermediate trans-5OH (T1) - water and hydroxyl complexed at carbonyl Charge $=0$ Multiplicity $=3$

C, $0,-1.2011484241,2.1723739751,-0.6527132698$ C, $0,-1.9581595239,0.8741382083,-0.5958854914$ $\mathrm{C}, 0,-1.3484980451,-0.329460903,-0.3473827712$ C, $0,0.0839827392,-0.4105198921,-0.1751036034$ C,0,0.9170653164,0.8315434054,-0.4462978978 C, $0,0.1709291281,2.087696049,0.0051806664$ $\mathrm{H}, 0,-1.0959088438,2.4682100421,-1.7079183798$ $\mathrm{H}, 0,-1.8054967643,2.9570998066,-0.1805809642$ $\mathrm{H}, 0,1.0714015317,0.9147593442,-1.5364819608$ $\mathrm{H}, 0,0.0564649414,2.0561391748,1.0962818005$ $\mathrm{H}, 0,0.7605626577,2.9779549458,-0.237705772$ C,0,-2.1783434321,-1.6020303453,-0.3097493819 C,0,-3.4449073158,1.0129494446,-0.7763808268 $\mathrm{C}, 0,-4.2181817442,-0.2768023278,-0.8162577956$ C, $0,-3.4386945858,-1.4995669212,-1.1802971531$ $\mathrm{H}, 0,-1.5901251166,-2.4440471689,-0.6796008045$ $\mathrm{H}, 0,-4.0710606095,-2.3844850587,-1.0864140661$ $\mathrm{H}, 0,-2.4594012244,-1.8433284253,0.7233772467$ $\mathrm{H}, 0,-3.1350137061,-1.3879365017,-2.2299939668$ C, $0,2.2698880261,0.694541253,0.2354047183$ $\mathrm{H}, 0,2.0715772397,0.657617348,1.3191220766$ C, $0,0.7215921658,-1.6104598999,0.2451214577$ $\mathrm{H}, 0,0.0988156281,-2.4585950519,0.5168976407$ 
C, $0,2.0843866696,-1.7581218831,0.3287087836$

C, $0,2.9712379548,-0.6320374831,-0.1059001999$

C, $0,4.2792433147,-0.4354114373,0.6708301693$

C, $0,3.3482710846,1.760320624,0.0018335007$

$\mathrm{H}, 0,3.3592569938,2.0714292034,-1.0492047484$

$\mathrm{H}, 0,3.1771875513,2.6551909338,0.605388888$

$\mathrm{C}, 0,4.6687647367,1.0343989215,0.3812380788$

$\mathrm{H}, 0,5.158440051,1.4872468723,1.2471709054$

$\mathrm{H}, 0,5.3846046475,1.0522219894,-0.4468744676$

C, $0,3.268145581,-0.7839338243,-1.6114450114$

$\mathrm{H}, 0,3.7810979456,-1.7319394789,-1.7972796563$

$\mathrm{H}, 0,2.3393365671,-0.7908385843,-2.1885689201$

$\mathrm{H}, 0,3.9040475561,0.0239377755,-1.9887974872$

$0,0,-5.4175804685,-0.3022750699,-0.5599389646$

$0,0,5.2684099085,-1.3824510784,0.2798204861$

$\mathrm{H}, 0,2.5188168661,-2.7050094599,0.6417166833$

$\mathrm{H}, 0,-3.8709516801,1.6496624616,0.0112629307$

$\mathrm{H}, 0,-3.6565228524,1.5353476709,-1.7234879281$

$\mathrm{O}, 0,-1.7931238517,0.5089405711,2.2164481811$

$\mathrm{H}, 0,4.0682635539,-0.5651967878,1.7411836469$

$\mathrm{H}, 0,6.0455713303,-1.2564161281,0.8408026397$

$\mathrm{H}, 0,-2.7168105476,0.1901190381,2.0576816021$

$0,0,-4.4484431501,-0.3935658609,2.2434270784$

$\mathrm{H}, 0,-5.106724626,-0.1472266249,1.5770497987$

$\mathrm{H}, 0,-4.7794601748,-0.0334498615,3.0774565089$

hydration TS2 trans-5OH (T1)

Charge $=0$ Multiplicity $=3$

C, $0,-1.1665225109,2.2911090104,-0.3000993521$

C, $0,-1.9746938901,1.0236500355,-0.3584419538$

C, $0,-1.3639962009,-0.2221867432,-0.4286764925$

C, $0,0.0969882623,-0.3514061714,-0.2019428881$

C, $0,0.9404354563,0.9038743127,-0.3158182923$

C, $0,0.2146766622,2.0991387149,0.3099697425$

$\mathrm{H}, 0,-1.0751623744,2.6633832512,-1.3309417212$

$\mathrm{H}, 0,-1.7382885551,3.0438837635,0.2527025719$

$\mathrm{H}, 0,1.0844871265,1.1309794333,-1.3867966186$

$\mathrm{H}, 0,0.1240638433,1.933585224,1.3897925171$

$\mathrm{H}, 0,0.8110406744,3.00576648,0.1641168002$

C, $0,-2.1634317529,-1.4761163772,-0.6485273613$

C, $0,-3.4163095707,1.2281732054,-0.7550046424$

C, $0,-4.2299147277,-0.0342902014,-0.7151889506$

C, $0,-3.5506129712,-1.251280155,-1.2676853746$

$\mathrm{H}, 0,-1.5970541235,-2.157292082,-1.2901757533$

$\mathrm{H}, 0,-4.1894784463,-2.1261602355,-1.133378337$

$\mathrm{H}, 0,-2.2713584055,-1.9887111787,0.3172498274$

$\mathrm{H}, 0,-3.4328724382,-1.0658859168,-2.3437051331$

C, $0,2.2936265348,0.6632901954,0.3375994343$

$\mathrm{H}, 0,2.0977046083,0.523177506,1.4133377061$

C, $0,0.6684926711,-1.5796486474,0.0934261686$ 
$\mathrm{H}, 0,0.0225448808,-2.4422841293,0.2345415543$ C, $0,2.0557794456,-1.7795706786,0.1932987533$ $\mathrm{C}, 0,2.9615565209,-0.6390899117,-0.1386341822$ $\mathrm{C}, 0,4.2831481282,-0.5492382807,0.634543853$ C, $0,3.3955722151,1.7222420628,0.202544576$ $\mathrm{H}, 0,3.403388054,2.1393525469,-0.8109494887$ $\mathrm{H}, 0,3.2513003699,2.5534928646,0.8970157346$ C, $0,4.7033152553,0.9328242483,0.4878191335$ $\mathrm{H}, 0,5.2133254271,1.2854246224,1.3878483131$ $\mathrm{H}, 0,5.408575635,1.0182248737,-0.3450097479$ C,0,3.2325222132,-0.6470186685,-1.6581975847 $\mathrm{H}, 0,3.733795149,-1.5756197714,-1.945060807$ $\mathrm{H}, 0,2.2944977604,-0.5866435928,-2.2173382446$ $\mathrm{H}, 0,3.8695742806,0.1902553551,-1.9622769128$ $0,0,-5.3746635805,-0.0511432333,-0.2793609851$ $\mathrm{O}, 0,5.2431059806,-1.4751290601,0.1365863207$ $\mathrm{H}, 0,2.4566066573,-2.7673808745,0.4021390733$ $\mathrm{H}, 0,-3.8794948883,1.9893812292,-0.1222873044$ $\mathrm{H}, 0,-3.4438541599,1.5976865439,-1.7920980076$ $\mathrm{O}, 0,-2.2082617557,0.7784958743,1.684125118$ $\mathrm{H}, 0,4.081416757,-0.779659963,1.6893806198$ $\mathrm{H}, 0,6.0161331874,-1.4496016429,0.7162134907$ $\mathrm{H}, 0,-2.7935069908,-0.0020353483,1.7417444348$ $\mathrm{O}, 0,-4.1448836075,-1.4714217756,2.0252996661$ $\mathrm{H}, 0,-4.9209128309,-1.1724449282,1.5290263129$ $\mathrm{H}, 0,-4.4120539756,-1.445025786,2.9536714135$

diol trans-5OH (T1) - water complexed between $\mathrm{C}_{5} \mathrm{OH}$ and $\mathrm{C}_{3}$ carbonyl Charge $=0$ Multiplicity $=1$

C, $0,-1.1332898064,2.2527647556,-0.1851678244$

C,0,-2.0008081758,1.0132009687,0.0480156639

$\mathrm{C}, 0,-1.3442910331,-0.2139269642,-0.5382172219$

C, $0,0.1161684605,-0.3825547304,-0.2856608165$

C, $0,0.9765550179,0.8612694955,-0.4178118835$

C, $0,0.302132096,2.0519579181,0.2837159122$

$\mathrm{H}, 0,-1.1415672844,2.4750313135,-1.2588658505$

$\mathrm{H}, 0,-1.599422216,3.0999379697,0.3314852532$

$\mathrm{H}, 0,1.0613623939,1.1171844184,-1.4890593006$

$\mathrm{H}, 0,0.3225819792,1.882483608,1.3676884125$

$\mathrm{H}, 0,0.8837226073,2.9595832417,0.0881303916$

C, $0,-2.1699530392,-1.4246559384,-0.835261435$

C, $0,-3.404799103,1.2613699048,-0.5417936016$

C, $0,-4.2177945453,0.0017222948,-0.6472111166$

C,0,-3.5635294151,-1.1130869253,-1.4112157025

$\mathrm{H}, 0,-1.6319717708,-2.0852402889,-1.523122915$

$\mathrm{H}, 0,-4.2033681633,-1.9975248962,-1.3969771876$

$\mathrm{H}, 0,-2.3036006937,-2.0058590001,0.0937328097$

$\mathrm{H}, 0,-3.4553021676,-0.7700450705,-2.4477228115$

C, $0,2.3595491147,0.5973319607,0.1622932151$

$\mathrm{H}, 0,2.2227201963,0.4911273541,1.2509033541$ 
C, $0,0.6542270924,-1.5983601593,0.05437421$

$\mathrm{H}, 0,-0.0128315533,-2.4422984123,0.2167310824$

C,0,2.0599487629,-1.8395280053,0.1248055011

C, $0,2.96735685,-0.7354851827,-0.3091294749$

C,0,4.3384976332,-0.650340018,0.3727630653

C, $0,3.4751944957,1.6270197834,-0.0657799392$

$\mathrm{H}, 0,3.4259532401,2.0240548231,-1.0861813874$

$\mathrm{H}, 0,3.3942541711,2.4752306578,0.6186418948$

C,0,4.7817614404,0.8147518437,0.1501759532

$\mathrm{H}, 0,5.3644380193,1.1810902192,0.9992515946$

$\mathrm{H}, 0,5.4266869858,0.8577231499,-0.7334728996$

C,0,3.1345784335,-0.7945891386,-1.8424168238

$\mathrm{H}, 0,3.5917133436,-1.7442381902,-2.1344131117$

$\mathrm{H}, 0,2.1611157211,-0.7264567996,-2.3366982219$

$\mathrm{H}, 0,3.7684912151,0.0165771277,-2.2166818336$

$\mathrm{O}, 0,-5.3298096999,-0.105587314,-0.1403978319$

$\mathrm{O}, 0,5.2450625252,-1.6149920841,-0.15258016$

$\mathrm{H}, 0,2.439021743,-2.8418280047,0.3011464542$

$\mathrm{H}, 0,-3.9299539061,2.0062912846,0.0614106741$

$\mathrm{H}, 0,-3.2872046984,1.6460524067,-1.5629228753$

$\mathrm{O}, 0,-2.1039664708,0.8586181708,1.4791669925$

$\mathrm{H}, 0,4.1996544286,-0.84178865,1.4457320758$

$\mathrm{H}, 0,6.0647485528,-1.567893337,0.357592585$

$\mathrm{H}, 0,-2.6550181828,0.0771949669,1.6883206069$

$0,0,-3.8233245763,-1.2898882702,2.1578121381$

$\mathrm{H}, 0,-4.6806756959,-1.0906197413,1.7537290274$

$\mathrm{H}, 0,-3.9801084626,-1.269175966,3.1115627888$

diol trans- $5 \mathrm{OH}(\mathrm{SO})$ - water complexed between $\mathrm{C}_{5} \mathrm{OH}$ and $\mathrm{C}_{3}$ carbonyl

Charge $=0$ Multiplicity $=1$

C,0,-1.1005227154,2.1742752957,-0.224567145

C, $0,-1.9700689094,0.9558851231,0.0709910888$

C, $0,-1.2404029085,-0.370165893,-0.1549119656$

C, $0,0.0902541307,-0.4304672651,-0.3998441838$

C, $0,0.9666721429,0.8255201932,-0.4315538227$

C, $0,0.3104444038,1.9850790688,0.3081049053$

$\mathrm{H}, 0,-1.0532350132,2.3217346874,-1.3101384971$

$\mathrm{H}, 0,-1.5927616993,3.0519558088,0.2069249871$

$\mathrm{H}, 0,1.0951241345,1.1325860215,-1.4812935275$

$\mathrm{H}, 0,0.2883743075,1.7733990349,1.3858332589$

$\mathrm{H}, 0,0.8992440494,2.8971069431,0.1651029943$

C, $0,-2.1397830464,-1.5742285198,0.0219791423$

C, $0,-3.238343476,1.0325958268,-0.8043704319$

C, $0,-4.1156250262,-0.185592568,-0.7069677336$

C, $0,-3.4116566356,-1.5059846932,-0.8452453752$

$\mathrm{H}, 0,-1.6301894736,-2.5109065266,-0.194659166$

$\mathrm{H}, 0,-4.1045718798,-2.3145293317,-0.6016728289$

$\mathrm{H}, 0,-2.4450748555,-1.6276777433,1.0750153389$

$\mathrm{H}, 0,-3.128980467,-1.596680607,-1.9029846572$

C, $0,2.3310065944,0.4647322747,0.1386981102$ 
$\mathrm{H}, 0,2.1483801803,0.040471057,1.1399052857$

C, $0,0.7940086689,-1.716572417,-0.6058544067$

$\mathrm{H}, 0,0.1963891269,-2.6183736583,-0.6838563462$

C, $0,2.1293041393,-1.8385834311,-0.716037539$

C, $0,3.0304584636,-0.6414015619,-0.6648964385$

C, $0,4.3181218885,-0.7700503194,0.1614081079$

C, $0,3.4134910538,1.5374994678,0.3011320198$

$\mathrm{H}, 0,3.4590135256,2.1790970649,-0.5864776657$

$\mathrm{H}, 0,3.2288636596,2.1828672236,1.1636218079$

C, $0,4.7150135462,0.7007638901,0.453137218$

$\mathrm{H}, 0,5.1503967034,0.7947320573,1.4512687789$

$\mathrm{H}, 0,5.4773800786,1.0116490937,-0.2682020117$

C, $0,3.3830072476,-0.2326655514,-2.1083260741$

$\mathrm{H}, 0,3.852117926,-1.0738358969,-2.6274204449$

$\mathrm{H}, 0,2.4843122217,0.0387834908,-2.6681127001$

$\mathrm{H}, 0,4.081104375,0.6098322841,-2.1397091207$

$0,0,-5.3286603774,-0.0993942601,-0.5498403442$

$0,0,5.3159509813,-1.5110933789,-0.5335826238$

$\mathrm{H}, 0,2.5690227304,-2.8181909174,-0.9014904721$

$\mathrm{H}, 0,-3.8117150534,1.9299385482,-0.5539672574$

$\mathrm{H}, 0,-2.9241163304,1.0969849971,-1.854815298$

O,0,-2.3400135924,1.0614836168,1.4575159316

$\mathrm{H}, 0,4.0803524608,-1.2897987837,1.0990613833$

$\mathrm{H}, 0,6.0603494771,-1.6527369243,0.0666282252$

$\mathrm{H}, 0,-3.0330818156,0.4138757572,1.6934253195$

$0,0,-4.495188066,-0.6075114373,2.2590424939$

$\mathrm{H}, 0,-5.2671112937,-0.4037759892,1.7114834222$

H, $0,-4.7321497229,-0.3190246017,3.1509376879$

\section{Structures for acidic reversion}

\section{$17 \alpha$}

cis- $12 \mathrm{OH}(\mathrm{SO})$ - complex with $\mathrm{H}_{3} \mathrm{O}^{+}$at $\mathrm{C}_{12}$

Charge $=1$ Multiplicity $=1$

C, $0,-2.2864785959,-2.2079627067,-0.2418140297$

C, $0,-2.8439052018,-0.8120492924,-0.1221573743$

C, $0,-2.0700809944,0.2902888562,-0.0314274784$

C, $0,-0.5930140611,0.1747495894,-0.0370123693$

$\mathrm{C}, 0,-0.0193146461,-1.2173765906,0.1699253661$

C, $0,-0.8198808234,-2.2265632908,-0.653525674$

$\mathrm{H}, 0,-2.4131002419,-2.7140376396,0.7266910535$

$\mathrm{H}, 0,-2.8940533066,-2.7728991958,-0.9584777743$

$\mathrm{H}, 0,-0.1523634442,-1.4774258543,1.2334579812$

$\mathrm{H}, 0,-0.7280199684,-1.9674067715,-1.7167551283$

$\mathrm{H}, 0,-0.4025837839,-3.2307184456,-0.5238348549$

C, $0,-2.6833362942,1.67076718,0.0824410786$

C, $0,-4.3511422592,-0.7524683433,-0.1398422127$

C, $0,-4.9475058258,0.6221505378,0.0065804906$

C, $0,-4.0887123407,1.6379020437,0.6946511698$

$\mathrm{H}, 0,-2.0553803051,2.3040046911,0.7148288245$ 
$\mathrm{H}, 0,-4.5725234658,2.6159929969,0.6620122603$ $\mathrm{H}, 0,-2.7173237396,2.152446079,-0.9031661565$ $\mathrm{H}, 0,-4.0089821956,1.3182025815,1.7427758416$ $\mathrm{H}, 0,-4.7422197972,-1.2077323915,-1.0575062235$ C,0,1.4664444147,-1.2445941666,-0.1601444796 $\mathrm{H}, 0,1.5667626502,-1.1400261443,-1.25216183$ C, $0,0.2035612958,1.2453481616,-0.2124882042$ $\mathrm{H}, 0,-0.2224924999,2.23228338,-0.3872760328$ $\mathrm{C}, 0,1.7100387001,1.1857316945,-0.2531150873$ $\mathrm{H}, 0,2.0329131949,1.152960923,-1.3032420159$ C,0,2.223969356,-0.0576229244,0.4699714656 C,0,3.6951568792,-0.45831599,0.2488541228 C, $0,2.2588361198,-2.4823077783,0.2812904135$ $\mathrm{H}, 0,1.8644090796,-2.8631772908,1.2298876344$ $\mathrm{H}, 0,2.1887758223,-3.291959542,-0.4484472447$ C,0,3.7153981323,-1.9822559744,0.459198921 $\mathrm{H}, 0,4.4068881266,-2.4435009583,-0.2524294929$ $\mathrm{H}, 0,4.0921860871,-2.2067445687,1.4599637708$ C,0,2.0071104135,0.045626702,1.9859341078 $\mathrm{H}, 0,2.672199609,0.8013376439,2.4126392489$ $\mathrm{H}, 0,0.9785252619,0.3240033093,2.2269113996$ $\mathrm{H}, 0,2.2237895908,-0.9074784641,2.4781665288$ $\mathrm{O}, 0,-6.0785726249,0.8688844088,-0.3934255011$ $\mathrm{H}, 0,-4.7502390472,-1.3593268769,0.6882476093$ $\mathrm{O}, 0,2.2919833947,2.3815377215,0.3279492785$ $\mathrm{H}, 0,1.7485029118,3.1518081465,0.0957423202$ $\mathrm{H}, 0,3.5849470689,2.5252707792,-0.3820304825$ $0,0,4.4617445692,2.3216747212,-0.9106938912$ $\mathrm{H}, 0,4.4028092235,1.2788478404,-1.0995141392$ $\mathrm{H}, 0,4.445229449,2.7975322176,-1.7584888672$ $\mathrm{H}, 0,4.3718785932,0.0702044282,0.9288447184$ $\mathrm{O}, 0,4.0913423877,-0.1167060414,-1.1054305546$ $\mathrm{H}, 0,4.8869891308,-0.621230391,-1.3388935064$

TS1 cis- $12 \mathrm{OH}(\mathrm{S} 0)$ - complex

Charge $=1$ Multiplicity $=1$

C, $0,-2.384509,-2.241246,-0.009897$

C, $0,-2.914467,-0.838279,-0.065869$

$\mathrm{C}, 0,-2.115105,0.259977,-0.125463$

$\mathrm{C}, 0,-0.667653,0.104536,-0.099366$

C, $0,-0.090532,-1.264462,0.202983$

C, $0,-0.939311,-2.34277,-0.469339$

$\mathrm{H}, 0,-2.482286,-2.587274,1.030529$

$\mathrm{H}, 0,-3.034681,-2.888898,-0.607496$

$\mathrm{H}, 0,-0.160256,-1.409667,1.293818$

$\mathrm{H}, 0,-0.883136,-2.214153,-1.557703$

$\mathrm{H}, 0,-0.53132,-3.329329,-0.229745$

$\mathrm{C}, 0,-2.703716,1.655085,-0.186114$

C, $0,-4.413165,-0.74134,-0.06952$

C, $0,-4.987307,0.652097,-0.036484$ 
C,0,-4.081996, 1.727113,0.479993

$\mathrm{H}, 0,-2.044592,2.359404,0.32668$

$\mathrm{H}, 0,-4.550546,2.70311,0.341789$

$\mathrm{H}, 0,-2.773925,1.989261,-1.228574$

$\mathrm{H}, 0,-3.964775,1.542091,1.556819$

$\mathrm{H}, 0,-4.816986,-1.272176,-0.940176$

C, $0,1.373185,-1.326464,-0.213158$

$\mathrm{H}, 0,1.408406,-1.267834,-1.310798$

$\mathrm{C}, 0,0.158321,1.164204,-0.422255$

$\mathrm{H}, 0,-0.261234,2.077288,-0.831476$

C, $0,1.563741,1.040271,-0.393303$

$\mathrm{H}, 0,2.145138,1.564437,-1.14544$

C,0,2.158381,-0.103315,0.347192

$\mathrm{C}, 0,3.612693,-0.471136,0.025216$

$\mathrm{C}, 0,2.216522,-2.527312,0.228961$

$\mathrm{H}, 0,1.896659,-2.874873,1.216636$

$\mathrm{H}, 0,2.106691,-3.363899,-0.464217$

C,0,3.673102,-1.992621,0.278429

$\mathrm{H}, 0,4.312933,-2.461305,-0.474874$

$\mathrm{H}, 0,4.12696,-2.187063,1.253401$

C, $0,2.000949,0.016664,1.873143$

$\mathrm{H}, 0,2.73852,0.719185,2.264682$

$\mathrm{H}, 0,1.008656,0.375559,2.153932$

$\mathrm{H}, 0,2.164698,-0.953871,2.349306$

O,0,-6.141183,0.861199,-0.384114

$\mathrm{H}, 0,-4.806863,-1.274768,0.809383$

$0,0,2.231115,2.708074,0.826026$

$\mathrm{H}, 0,1.724602,3.44463,0.451895$

$\mathrm{H}, 0,3.125761,2.774381,0.418092$

$0,0,4.621889,2.510048,-0.541728$

$\mathrm{H}, 0,4.463455,1.671482,-1.015622$

$\mathrm{H}, 0,4.69496,3.186069,-1.228743$

$\mathrm{H}, 0,4.31492,0.080129,0.661805$

$0,0,3.869098,-0.134351,-1.342583$

$\mathrm{H}, 0,4.676094,-0.587696,-1.625592$

trans-5OH(SO) - complex with $\mathrm{H}_{3} \mathrm{O}^{+}$at $\mathrm{C}_{5}$

Charge $=1$ Multiplicity $=1$

C, $0,-1.0961556789,2.1225691241,-0.5566558087$

C,0,-1.9501458212,0.9172148711,-0.1828312922

C, $0,-1.1946752209,-0.4053906668,-0.2009488609$

C, $0,0.149379366,-0.4496468817,-0.3682206947$

C, $0,1.0017187069,0.8166780809,-0.4855894895$

C, $0,0.2819626403,2.0355621201,0.0757206836$

$\mathrm{H}, 0,-0.9932858011,2.13997849,-1.6477827877$

$\mathrm{H}, 0,-1.6312324486,3.0355407277,-0.2702760946$

$\mathrm{H}, 0,1.1847733657,1.0036549681,-1.5551889155$

$\mathrm{H}, 0,0.1995956711,1.9517285703,1.1676080317$

$\mathrm{H}, 0,0.8574689811,2.9404033469,-0.1427402305$

C, $0,-2.0745622672,-1.6178753098,0.0211056033$ 
C,0,-3.2033089988,0.8770850944,-1.0738644194

C, $0,-4.0428990776,-0.3555256718,-0.8625941239$

C,0,-3.2947082194,-1.6554984428,-0.9206407183

$\mathrm{H}, 0,-1.5255613942,-2.5494468753,-0.0960170253$

$\mathrm{H}, 0,-3.9665860613,-2.4771742892,-0.6646041671$

$\mathrm{H}, 0,-2.4373817457,-1.6089386555,1.0563890467$

$\mathrm{H}, 0,-2.9476928135,-1.7877325319,-1.9534176011$

C, $0,2.3362861178,0.553233879,0.1979502178$

$\mathrm{H}, 0,2.1056794693,0.231209867,1.2249277572$

C,0,0.8880915574,-1.7318196805,-0.4183426197

$\mathrm{H}, 0,0.3156557953,-2.6517856514,-0.4542865924$

C,0,2.229527473,-1.8296639999,-0.4361048435

C,0,3.1009370378,-0.6114516775,-0.4499806551

C, $0,4.3780900406,-0.6322153284,0.403162302$

C, $0,3.3889520446,1.6583207444,0.2969449784$

$\mathrm{H}, 0,3.4705639088,2.201945674,-0.6514903852$

$\mathrm{H}, 0,3.1540414196,2.3893319622,1.0746585268$

C,0,4.6975559723,0.8728733959,0.601793012

$\mathrm{H}, 0,5.0375776239,1.0323893862,1.6295698111$

$\mathrm{H}, 0,5.5117272901,1.182408249,-0.0590839997$

C, $0,3.5269811562,-0.342415998,-1.9079801044$

$\mathrm{H}, 0,4.0503372753,-1.2211222296,-2.2995137852$

$\mathrm{H}, 0,2.6629708139,-0.1572777522,-2.5507258786$

$\mathrm{H}, 0,4.2069196209,0.5118490527,-1.9852249223$

$0,0,-5.2465761777,-0.2881580849,-0.6499909069$

$0,0,4.0862006211,-1.2833582435,1.6432909275$

$\mathrm{H}, 0,2.6961801228,-2.8117127752,-0.5033126501$

$\mathrm{H}, 0,-3.8030557141,1.7780394355,-0.9137330398$

$\mathrm{H}, 0,-2.866990197,0.8582824603,-2.1180482562$

$\mathrm{O}, 0,-2.3881510836,1.1226489095,1.2149385614$

$\mathrm{H}, 0,-2.7250544729,2.0318223783,1.3035898734$

$\mathrm{H}, 0,5.1951136247,-1.1673477654,-0.0960345106$

$\mathrm{H}, 0,4.8649062963,-1.2094265136,2.2115443696$

$\mathrm{H}, 0,-3.2465178165,0.3846975689,1.8621890459$

$\mathrm{O}, 0,-3.9807376866,-0.1971142453,2.480005694$

$\mathrm{H}, 0,-4.8277925809,-0.2782010055,2.0053103445$

$\mathrm{H}, 0,-4.1587197356,0.2641969194,3.318561592$

TS1 trans-5OH(S0) - complex
Charge $=1$ Multiplicity $=1$
C, $0,-1.069065,2.127533,-0.594407$
C, $0,-1.86493,0.871975,-0.4747$
C, $0,-1.215211,-0.404905,-0.32733$
C, $0,0.162364,-0.459406,-0.315337$
$\mathrm{C}, 0,1.012634,0.797729,-0.466406$
C, $0,0.28519,2.019818,0.077209$
$\mathrm{H}, 0,-0.939719,2.279723,-1.678378$
$\mathrm{H}, 0,-1.666494,2.969382,-0.234112$
$\mathrm{H}, 0,1.167283,0.951113,-1.547218$
$\mathrm{H}, 0,0.16413,1.927639,1.163471$ 
$\mathrm{H}, 0,0.87286,2.920182,-0.121132$

C, $0,-2.108159,-1.613005,-0.12235$

$\mathrm{C}, 0,-3.241542,0.949778,-1.068001$

$\mathrm{C}, 0,-4.097141,-0.264145,-0.812813$

$\mathrm{C}, 0,-3.39629,-1.578663,-0.960844$

$\mathrm{H}, 0,-1.588942,-2.538784,-0.360912$

$\mathrm{H}, 0,-4.069361,-2.389521,-0.676545$

$\mathrm{H}, 0,-2.380149,-1.663012,0.939623$

$\mathrm{H}, 0,-3.139336,-1.685495,-2.022902$

C,0,2.361929,0.571535,0.197119

$\mathrm{H}, 0,2.161712,0.344365,1.2545$

C, $0,0.86228,-1.737708,-0.182156$

$\mathrm{H}, 0,0.275126,-2.640716,-0.072844$

C, $0,2.207818,-1.850762,-0.188645$

$\mathrm{C}, 0,3.085558,-0.657232,-0.372179$

C,0,4.402013,-0.617381,0.416184

C, $0,3.427904,1.667099,0.15637$

$\mathrm{H}, 0,3.464589,2.13412,-0.834424$

$\mathrm{H}, 0,3.237823,2.458097,0.885567$

C, $0,4.74547,0.893338,0.454993$

$\mathrm{H}, 0,5.150631,1.142964,1.440032$

$\mathrm{H}, 0,5.518275,1.127528,-0.281874$

C, $0,3.426144,-0.543568,-1.873333$

$\mathrm{H}, 0,3.94097,-1.453111,-2.19848$

$\mathrm{H}, 0,2.525898,-0.430962,-2.482109$

$\mathrm{H}, 0,4.08858,0.304839,-2.070094$

$0,0,-5.276391,-0.154424,-0.512468$

$0,0,4.153582,-1.143731,1.722188$

$\mathrm{H}, 0,2.660907,-2.837789,-0.10757$

$\mathrm{H}, 0,-3.753184,1.865943,-0.76652$

$\mathrm{H}, 0,-3.079218,0.998881,-2.157603$

$0,0,-2.43957,1.14864,1.479447$

$\mathrm{H}, 0,-2.891196,2.00708,1.522374$

$\mathrm{H}, 0,5.187084,-1.21124,-0.066707$

$\mathrm{H}, 0,4.958333,-1.040002,2.248012$

$\mathrm{H}, 0,-3.110184,0.485536,1.789543$

$0,0,-4.306527,-0.618069,2.346792$

$\mathrm{H}, 0,-5.039677,-0.624104,1.713479$

$\mathrm{H}, 0,-4.687287,-0.294159,3.174913$

common int1(SO) - complex with two $\mathrm{H}_{2} \mathrm{O}$ at $\mathrm{C}_{4}$

$\mathrm{C}, 0,-0.8500098394,2.5268978159,0.2284340449$

$\mathrm{C}, 0,-1.6915596315,1.4015571431,-0.2720706231$

$\mathrm{C}, 0,-1.1728069493,0.1731255688,-0.6342621117$

C, $0,0.2270623238,-0.0505397153,-0.4868624463$

C, $0,1.149490405,1.0832103656,-0.1049422145$

C, $0,0.4543135619,2.0537508805,0.8438076037$

$\mathrm{H}, 0,-0.6459896737,3.1656453111,-0.6473533542$

$\mathrm{H}, 0,-1.4363278904,3.1347845953,0.9227353982$

$\mathrm{H}, 0,1.3511826312,1.6238446758,-1.0478354094$ 
$\mathrm{H}, 0,0.2577711611,1.5497443526,1.7984482981$ $\mathrm{H}, 0,1.1121293105,2.9039997291,1.0411769086$ C,0,-2.0726898488,-0.9327414883,-1.1547742678 C,0,-3.1523790754,1.7065344759,-0.3521555761 C, $0,-4.032899723,0.5764191621,-0.8115417557$ C, $0,-3.3886399636,-0.4053557531,-1.7368300806$ $\mathrm{H}, 0,-1.5628472937,-1.4832888024,-1.9475380576$ $\mathrm{H}, 0,-4.078675413,-1.2206385145,-1.9609119234$ $\mathrm{H}, 0,-2.2813043173,-1.6484654306,-0.350174311$ $\mathrm{H}, 0,-3.1732722187,0.1417585845,-2.6649967501$ $\mathrm{H}, 0,-3.5001166926,2.0824999421,0.616538747$ C, $0,2.4483464167,0.5298628039,0.4555638024$ $\mathrm{H}, 0,2.1863413336,-0.0144421201,1.3747352903$ $\mathrm{C}, 0,0.7664165258,-1.3556772391,-0.7241375781$ $\mathrm{H}, 0,0.0925831518,-2.1748165831,-0.9419035706$ C,0,2.10952107,-1.5975343327,-0.7121152457 $\mathrm{H}, 0,2.4692545264,-2.600688031,-0.9373722206$ C, $0,3.0862837607,-0.5044752526,-0.481611289$ C, $0,4.3692788939,-0.8563175403,0.283148315$ $\mathrm{H}, 0,5.1173528964,-1.3261143188,-0.3653411633$ C, $0,3.6064783761,1.4697341942,0.7885588578$ $\mathrm{H}, 0,3.7251762488,2.2274298669,0.0061993676$ $\mathrm{H}, 0,3.4552116793,1.9923280151,1.7354699312$ $\mathrm{C}, 0,4.8366460119,0.5158624639,0.8311605839$ $\mathrm{H}, 0,5.2115483691,0.3814664363,1.8497188003$ $\mathrm{H}, 0,5.6612178031,0.906382018,0.2294249869$ C, $0,3.4842242776,0.0542185598,-1.8683037607$ $\mathrm{H}, 0,3.9314079849,-0.7435134776,-2.4682735408$ $\mathrm{H}, 0,2.6207553021,0.4459112641,-2.4107878366$ $\mathrm{H}, 0,4.2236226579,0.8529155101,-1.7615538421$ $\mathrm{O}, 0,4.0123656237,-1.7622074381,1.3286249985$ $\mathrm{H}, 0,4.7982789077,-1.9369461676,1.8646034057$ $0,0,-5.2013640325,0.5059533234,-0.4573469007$ $\mathrm{H}, 0,-3.2822078627,2.5414860294,-1.0605363497$ $0,0,-3.1455721985,-0.5321491887,2.0585815276$ $\mathrm{H}, 0,-3.6778884455,-1.1629133098,1.5352974735$ $\mathrm{H}, 0,-2.2253954241,-0.7183156253,1.8312415394$ $0,0,-4.8915947308,-2.1930788387,0.5773418078$ $\mathrm{H}, 0,-5.508287089,-2.6425815112,1.1704800385$ $\mathrm{H}, 0,-5.4032962075,-1.4800441986,0.1630228224$

TS2 common(S0) - complex
Charge $=1$ Multiplicity $=1$
C,0,-0.8430218116,2.2339406089,-0.432399437
C, $0,-1.6499251113,0.9813440894,-0.4083469486$
C, $0,-1.0493829397,-0.2978189882,-0.2745323615$
C, $0,0.3363056813,-0.4024624678,-0.2345529892$
C, $0,1.2119853948,0.8334159294,-0.3797379864$
C, $0,0.5237775161,2.0608505533,0.2045733185$
$\mathrm{H}, 0,-0.7331021586,2.4962947925,-1.4977631685$ 
$\mathrm{H}, 0,-1.4230003029,3.0420579037,0.0229794749$ $\mathrm{H}, 0,1.3373711698,1.0002899121,-1.46320793$ $\mathrm{H}, 0,0.4199830677,1.937127854,1.2900424033$ $\mathrm{H}, 0,1.1368156055,2.9482598327,0.0255005751$ C, $0,-2.0005207515,-1.473386802,-0.1715449725$ C, $0,-3.0705684033,1.1053880866,-0.4633552338$ C, $0,-3.8712276838,-0.0174399264,-0.9976989169$ C, $0,-3.1679783128,-1.3345458744,-1.150707405$ $\mathrm{H}, 0,-1.4991762072,-2.4185432294,-0.3697754776$ $\mathrm{H}, 0,-3.8931029891,-2.1433333727,-1.0335740329$ $\mathrm{H}, 0,-2.3942865814,-1.5241048537,0.852814551$ $\mathrm{H}, 0,-2.7958494561,-1.3652325197,-2.184289079$ $\mathrm{H}, 0,-3.37335814,0.9695738784,0.8019116993$ C $, 0,2.572218797,0.5701574293,0.2449943528$ $\mathrm{H}, 0,2.3966084188,0.363474113,1.3110056297$ C, $0,0.9844083183,-1.6938454353,-0.0733279363$ $\mathrm{H}, 0,0.3660875342,-2.5724412296,0.0625488648$ C, $0,2.3290759285,-1.8499277615,-0.0970406932$ $\mathrm{H}, 0,2.7517345366,-2.848943868,0.0000461619$ C, $0,3.2371095939,-0.6900168785,-0.3275630299$ C, $0,4.5795442465,-0.6834690531,0.4161260047$ $\mathrm{H}, 0,5.3281361995,-1.3087052845,-0.0844626134$ C, $0,3.6720701931,1.6283828921,0.1552972264$ $\mathrm{H}, 0,3.6917058906,2.0804107526,-0.8428112511$ $\mathrm{H}, 0,3.5318427024,2.4345392719,0.8789689502$ C, $0,4.9723460867,0.815425934,0.4229689694$ $\mathrm{H}, 0,5.4159457524,1.064236135,1.3914447262$ $\mathrm{H}, 0,5.7287286277,1.0150257484,-0.340532713$ C, $0,3.528951082,-0.6121081174,-1.841779389$ $\mathrm{H}, 0,4.0100514283,-1.5395453098,-2.1673703234$ $\mathrm{H}, 0,2.6119719017,-0.4855778259,-2.4223118017$ $\mathrm{H}, 0,4.2054694609,0.2163758166,-2.071902568$ O,0,4.3561557333,-1.1853157776,1.736038525 $\mathrm{H}, 0,5.1788697672,-1.0982343324,2.236772944$ $0,0,-5.0614206519,0.1292561429,-1.2723542444$ $\mathrm{H}, 0,-3.4667391902,2.0955423381,-0.6913941035$ $0,0,-3.8515709251,0.7745854622,2.0565531707$ $\mathrm{H}, 0,-4.5647241979,0.0872887158,1.9307842$ $\mathrm{H}, 0,-3.1427150406,0.3504507857,2.5664339375$ $0,0,-5.7131738925,-1.0247978766,1.4269937329$ $\mathrm{H}, 0,-6.5612550413,-0.9199794437,1.8801974739$ $\mathrm{H}, 0,-5.8879408458,-0.8105927506,0.4967547136$

trenbolone( $\mathrm{SO}$ - complex with $\mathrm{H}_{3} \mathrm{O}^{+}$and $\mathrm{H}_{2} \mathrm{O}$ at $\mathrm{C}_{3}$ carbonyl Charge $=1$ Multiplicity $=1$

C, $0,-0.2361652003,2.6712525181,0.0998509471$

C, $0,-1.2567272915,1.573955788,0.1641189506$

C, $0,-0.8435601537,0.1882442057,0.1345837169$

C, $0,0.4830816328,-0.1322805171,-0.0286320806$

C, $0,1.5400676141,0.9557406681,-0.1977734366$ 
C,0,1.1382344209,2.2166119422,0.5586374466

$\mathrm{H}, 0,-0.180759738,2.98513256,-0.953722746$

$\mathrm{H}, 0,-0.6011210818,3.5345575327,0.663364395$

$\mathrm{H}, 0,1.589041249,1.2070268304,-1.2699092739$

$\mathrm{H}, 0,1.1293531341,2.0059928721,1.6359493734$

$\mathrm{H}, 0,1.8719549728,3.0078884187,0.3794375634$

C, $0,-1.9373273929,-0.8300776239,0.3803935434$

C,0,-2.5904021978,1.9334894675,0.2274067544

C, $0,-3.606145308,0.9861171868,0.0146984879$

$\mathrm{C}, 0,-3.2622717113,-0.4363036452,-0.2676918924$

$\mathrm{H}, 0,-1.6646354861,-1.818095142,0.0144466036$

$\mathrm{H}, 0,-4.0710787038,-1.0893432227,0.0696062038$

$\mathrm{H}, 0,-2.0909959907,-0.9158168738,1.4641409298$

$\mathrm{H}, 0,-3.2056530387,-0.5255356788,-1.3624034753$

$\mathrm{H}, 0,-5.3935635858,-2.7931495807,1.0518895031$

C,0,2.8912185999,0.4176923629,0.2471028321

$\mathrm{H}, 0,2.7887730568,0.1428951142,1.307384756$

C, $0,0.9295227623,-1.527630189,-0.0381394546$

$\mathrm{H}, 0,0.1905375214,-2.3055879759,0.1121343129$

C,0,2.2123421014,-1.8957322517,-0.2375993326

$\mathrm{H}, 0,2.4703647777,-2.9533123029,-0.2645142876$

C, $0,3.2746629056,-0.874695938,-0.4897240974$

C,0,4.6679417732,-1.1468652265,0.0945032684

$\mathrm{H}, 0,5.2478909341,-1.8351989091,-0.5320972176$

C,0,4.139971213,1.2917080812,0.1176040165

$\mathrm{H}, 0,4.1354208932,1.8330223645,-0.8354344193$

$\mathrm{H}, 0,4.2094965678,2.0343501488,0.915939394$

C, $0,5.3067452256,0.2627995696,0.1664236302$

$\mathrm{H}, 0,5.887451737,0.3454739728,1.0900503788$

$\mathrm{H}, 0,6.0003654701,0.410110886,-0.6656827912$

C, $0,3.4251790288,-0.6981683712,-2.0147893204$

$\mathrm{H}, 0,3.7158845674,-1.6524559883,-2.4654258615$

$\mathrm{H}, 0,2.4861034031,-0.3813452667,-2.475172634$

$\mathrm{H}, 0,4.1973557343,0.0378906059,-2.2591769139$

$\mathrm{O}, 0,4.5006731074,-1.7233143655,1.3924799847$

$\mathrm{H}, 0,5.370684842,-1.7946525385,1.8080500478$

$\mathrm{O}, 0,-4.8299078295,1.3826157971,-0.0110841486$

$\mathrm{H}, 0,-2.874354417,2.9763210623,0.3294921833$

O,0,-6.1420541511,-2.7690673794,0.4397093866

$\mathrm{H}, 0,-6.4052990504,-1.1991993394,-0.1650280431$

$\mathrm{H}, 0,-5.9049133994,-3.3778953227,-0.2732070523$

$\mathrm{O}, 0,-6.5687952309,-0.2932190489,-0.5308537177$

$\mathrm{H}, 0,-7.4198155484,-0.001809612,-0.1729862774$

$\mathrm{H}, 0,-5.5471867391,0.6271863544,-0.2133961363$

\section{$17 \beta$}

cis- $12 \mathrm{OH}(\mathrm{SO})$ - complex with $\mathrm{H}_{3} \mathrm{O}^{+}$at $\mathrm{C}_{12}$

Charge $=1$ Multiplicity $=1$ 
C, $0,-2.3525946491,-2.2220306456,0.150796421$ C, $0,-2.9030506608,-0.820297717,0.0822987602$ C, $0,-2.1234935994,0.2739165787,-0.0482370325$ C, $0,-0.6499101484,0.1420122686,-0.1285178104$ C, $0,-0.0559536832,-1.2128634511,0.208661604$ C, $0,-0.9285518759,-2.322034798,-0.379623432$ $\mathrm{H}, 0,-2.3908314655,-2.5588934262,1.1972855577$ $\mathrm{H}, 0,-3.0173782763,-2.8920416977,-0.4066657204$ $\mathrm{H}, 0,-0.0660004362,-1.3275134394,1.305346601$ $\mathrm{H}, 0,-0.9325429817,-2.225762885,-1.473723754$ $\mathrm{H}, 0,-0.5030979192,-3.3015778762,-0.1380030419$ C, $0,-2.7249875486,1.6624188917,-0.119069091$ $\mathrm{C}, 0,-4.4087370571,-0.7492173065,0.143228276$ C, $0,-4.9924739671,0.636839218,0.0818065321$ C, $0,-4.100221254,1.742523729,0.5542593226$ $\mathrm{H}, 0,-2.0650986051,2.3780247379,0.3784502164$ $\mathrm{H}, 0,-4.5830118832,2.706962664,0.385053827$ $\mathrm{H}, 0,-2.802407623,1.9871133341,-1.1646704429$ $\mathrm{H}, 0,-3.9717673784,1.5971695365,1.6356487815$ $\mathrm{H}, 0,-4.8530989735,-1.3523141245,-0.6572792607$ C, $0,1.3787783653,-1.2911768097,-0.2861791066$ $\mathrm{H}, 0,1.3440040271,-1.2718752717,-1.3879869743$ C, $0,0.1358893529,1.1707090553,-0.4940299454$ $\mathrm{H}, 0,-0.3037769872,2.1292755371,-0.7652340622$ C, $0,1.6408361207,1.1291213185,-0.5938097355$ $\mathrm{H}, 0,1.9450831142,1.0907831104,-1.6497891678$ C, $0,2.2344726062,-0.0762847694,0.1453687734$ C, $0,3.6121623827,-0.5721155233,-0.3391432319$ $\mathrm{H}, 0,3.6673613632,-0.4554155376,-1.4286255773$ C, $0,2.1940833305,-2.5205952688,0.139685661$ $\mathrm{H}, 0,1.9342149287,-2.8096303445,1.1640173239$ $\mathrm{H}, 0,1.9918562917,-3.3799806552,-0.5032613037$ C, $0,3.666653762,-2.057320274,0.0564916933$ $\mathrm{H}, 0,4.2511172514,-2.6301322355,-0.6669984477$ $\mathrm{H}, 0,4.164805785,-2.1430229512,1.0273901311$ C, $0,2.2315756339,0.1415326901,1.6613164147$ $\mathrm{H}, 0,2.9543680932,0.9110647022,1.9425331504$ $\mathrm{H}, 0,1.2464122659,0.4645516295,2.009395276$ $\mathrm{H}, 0,2.4978804428,-0.7782563083,2.190839388$ $0,0,4.7105103743,0.1613305943,0.2432290461$ $\mathrm{H}, 0,5.5394865677,-0.3041299294,0.0486305067$ O,0,-6.1385145616,0.8265179214,-0.3064481781 $\mathrm{H}, 0,-4.7582213149,-1.1990310359,1.0859826263$ O,0,2.158945538,2.3692420117,-0.0402073858 $\mathrm{H}, 0,1.6482828532,3.1143836376,-0.3953856871$ $\mathrm{H}, 0,3.5260862718,2.5654785457,-0.34055947$ $0,0,4.5748169657,2.507029174,-0.47308338$ $\mathrm{H}, 0,4.762720254,1.4994155841,-0.2123402807$ $\mathrm{H}, 0,4.789082907,2.6469938111,-1.4100833707$ 
TS1 cis-12OH(S0) - complex

Charge $=1$ Multiplicity $=1$

C,0,-2.425066,-2.187022,0.450721

C, $0,-2.950643,-0.81036,0.166046$

$\mathrm{C}, 0,-2.152184,0.240273,-0.163848$

$\mathrm{C}, 0,-0.709456,0.057151,-0.238838$

$\mathrm{C}, 0,-0.114242,-1.251717,0.237477$

C, $0,-1.037646,-2.413344,-0.125599$

$\mathrm{H}, 0,-2.411121,-2.313947,1.544098$

$\mathrm{H}, 0,-3.136908,-2.925394,0.067515$

$\mathrm{H}, 0,-0.052214,-1.198195,1.337146$

$\mathrm{H}, 0,-1.096933,-2.494512,-1.218471$

$\mathrm{H}, 0,-0.614403,-3.34791,0.2541$

$\mathrm{C}, 0,-2.734982,1.613409,-0.432097$

C, $0,-4.444848,-0.687882,0.253899$

C, $0,-5.009054,0.694136,0.047246$

C, $0,-4.067175,1.83128,0.294311$

$\mathrm{H}, 0,-2.039886,2.384002,-0.091208$

$\mathrm{H}, 0,-4.53703,2.772672,0.004144$

$\mathrm{H}, 0,-2.86738,1.762024,-1.510814$

$\mathrm{H}, 0,-3.880666,1.849148,1.377051$

$\mathrm{H}, 0,-4.913709,-1.375766,-0.460013$

C, $0,1.280278,-1.420239,-0.341679$

$\mathrm{H}, 0,1.165658,-1.495141,-1.434196$

$\mathrm{C}, 0,0.09604,1.035666,-0.789406$

$\mathrm{H}, 0,-0.348667,1.901465,-1.268167$

C, $0,1.504931,0.940658,-0.80489$

$\mathrm{H}, 0,2.053741,1.463866,-1.583483$

C, $0,2.171761,-0.179936,-0.068611$

C,0,3.497942,-0.681872,-0.658688

$\mathrm{H}, 0,3.425586,-0.64921,-1.753342$

C,0,2.140322,-2.604918,0.113832

$\mathrm{H}, 0,1.991944,-2.802839,1.180362$

$\mathrm{H}, 0,1.876961,-3.515153,-0.429047$

C,0,3.594205,-2.144457,-0.171026

$\mathrm{H}, 0,4.0913,-2.769613,-0.915944$

$\mathrm{H}, 0,4.200184,-2.167802,0.739768$

$\mathrm{C}, 0,2.307944,0.108334,1.436204$

$\mathrm{H}, 0,3.075504,0.864687,1.609286$

$\mathrm{H}, 0,1.369363,0.475318,1.856816$

$\mathrm{H}, 0,2.599099,-0.800467,1.969235$

$\mathrm{O}, 0,4.601374,0.117515,-0.245812$

$\mathrm{H}, 0,5.402288,-0.217896,-0.672818$

$\mathrm{O}, 0,-6.179442,0.852103,-0.271181$

$\mathrm{H}, 0,-4.772192,-1.02717,1.248932$

$\mathrm{O}, 0,1.921369,2.691917,0.347315$

$\mathrm{H}, 0,1.593283,3.378934,-0.252259$

$\mathrm{H}, 0,2.905192,2.816616,0.388358$

$\mathrm{O}, 0,4.595017,2.764165,0.51236$

$\mathrm{H}, 0,4.708292,1.850323,0.178596$

$\mathrm{H}, 0,5.017413,3.341414,-0.137434$ 
trans-5OH(SO) - complex with $\mathrm{H}_{3} \mathrm{O}^{+}$at $\mathrm{C}_{5}$

Charge $=1$ Multiplicity $=1$

C, $0,-1.1846206927,2.0118456394,-0.8131522135$

C, $0,-2.0259939041,0.8505073178,-0.2993101253$

C, $0,-1.2474189767,-0.4494650533,-0.142770495$

C, $0,0.1026159784,-0.4816937835,-0.2523996038$

C, $0,0.9370380796,0.778428761,-0.4946515653$

C, $0,0.1736057905,2.0456674395,-0.1340658548$

$\mathrm{H}, 0,-1.0492195988,1.8806990382,-1.8927935654$

$\mathrm{H}, 0,-1.7475030137,2.9416475851,-0.6696890252$

$\mathrm{H}, 0,1.1731865576,0.8269386428,-1.5689196238$

$\mathrm{H}, 0,0.0562027158,2.1140508153,0.9556667136$

$\mathrm{H}, 0,0.7384696287,2.9246724758,-0.4594609201$

C, $0,-2.112817168,-1.6438379249,0.1969159207$

$\mathrm{C}, 0,-3.2719042,0.6848474123,-1.1859260328$

C, $0,-4.0804668116,-0.5454934008,-0.8662846251$

C, $0,-3.2952648949,-1.820487721,-0.7761858038$

$\mathrm{H}, 0,-1.5449794844,-2.5714002434,0.213275057$

$\mathrm{H}, 0,-3.9508078091,-2.633904819,-0.4589719389$

$\mathrm{H}, 0,-2.5151484288,-1.5133370154,1.2099053637$

$\mathrm{H}, 0,-2.9077858862,-2.0436500948,-1.7783540628$

C,0,2.2368880587,0.6373262261,0.2855728407

$\mathrm{H}, 0,1.9516335564,0.4387189638,1.331974637$

C, $0,0.8679263871,-1.740596026,-0.1072383124$

$\mathrm{H}, 0,0.3158706942,-2.671885803,-0.048893346$

C, $0,2.2104762301,-1.8041168033,-0.0488440063$

C, $0,3.0617881814,-0.5762634076,-0.1641533912$

C, $0,4.2423004708,-0.4466657372,0.8096990474$

$\mathrm{C}, 0,3.256960447,1.7798758551,0.314765302$

$\mathrm{H}, 0,3.3934699809,2.2034424428,-0.6867899601$

$\mathrm{H}, 0,2.9475376256,2.5928361371,0.9764754285$

C, $0,4.5527006725,1.0738310549,0.805953371$

$\mathrm{H}, 0,4.850733287,1.4119050834,1.8016744798$

$\mathrm{H}, 0,5.3925046139,1.2625783556,0.129960367$

C,0,3.579022695,-0.4772951094,-1.6128629689

$\mathrm{H}, 0,4.1287611278,-1.3870744078,-1.8711038332$

$\mathrm{H}, 0,2.7508551552,-0.3787855048,-2.319348881$

$\mathrm{H}, 0,4.2559922923,0.3717051849,-1.7496523456$

$0,0,-5.2894912567,-0.4925818411,-0.6817131364$

$\mathrm{O}, 0,5.3394216638,-1.2621394644,0.4126835192$

$\mathrm{H}, 0,2.6998284262,-2.7746239824,0.0281437616$

$\mathrm{H}, 0,-3.8979450036,1.5793198462,-1.1186708954$

$\mathrm{H}, 0,-2.9277535446,0.5736435362,-2.222003275$

$0,0,-2.4748062909,1.2195306893,1.0608740983$

$\mathrm{H}, 0,-2.7341120981,2.1577065199,1.0592426446$

$\mathrm{H}, 0,3.9091847176,-0.7620253354,1.8072613132$

$\mathrm{H}, 0,6.0018999674,-1.2409326773,1.1161453236$

$\mathrm{H}, 0,-3.3896061587,0.5974405247,1.7374430238$

$\mathrm{O}, 0,-4.1696704888,0.0981956235,2.3786708124$ 
$\mathrm{H}, 0,-5.0163246268,0.0443545831,1.8996645698$ $\mathrm{H}, 0,-4.3246606645,0.6148754021,3.1888132122$

TS1 trans-5OH(SO) - complex

Charge $=1$ Multiplicity $=1$

C,0,-1.1466290589,2.0144848616,-0.8718269305

C, $0,-1.9254409308,0.7759219344,-0.5845577061$

C, $0,-1.2594398629,-0.4529188965,-0.240116393$

C, $0,0.1184456898,-0.4778810961,-0.1957955422$

C, $0,0.9524999543,0.7653052412,-0.4834398386$

C, $0,0.1852729496,2.036849058,-0.1486221357$

$\mathrm{H}, 0,-0.9827064504,2.0015829942,-1.9616547936$

$\mathrm{H}, 0,-1.7690954769,2.8890261396,-0.6635763117$

$\mathrm{H}, 0,1.1568250673,0.7697204585,-1.5666477817$

$\mathrm{H}, 0,0.0269656146,2.1016409766,0.9347991692$

$\mathrm{H}, 0,0.7639839211,2.9116610226,-0.4569949882$

C,0,-2.1364293702,-1.6351461387,0.1214206505

C, $0,-3.2928924876,0.7415608737,-1.203209636$

C,0,-4.132218212,-0.4411645839,-0.7926127092

C, $0,-3.4067434675,-1.7491088996,-0.7371274404$

$\mathrm{H}, 0,-1.5974284972,-2.5759558241,0.0334159281$

$\mathrm{H}, 0,-4.0724063566,-2.522832683,-0.350347221$

$\mathrm{H}, 0,-2.4307026528,-1.5320811192,1.1735213512$

$\mathrm{H}, 0,-3.1263788266,-2.0028584543,-1.7677977708$

C, $0,2.2702784421,0.6590278564,0.2677938885$

$\mathrm{H}, 0,2.0169609407,0.5533035457,1.3348524563$

C,0,0.8395944378,-1.7148315805,0.1067595262

$\mathrm{H}, 0,0.2681579263,-2.6150235784,0.294580027$

C,0,2.1865581246,-1.7927096557,0.1605519859

C, $0,3.0518246036,-0.6057003178,-0.1084736731$

C, $0,4.2821716511,-0.4215049244,0.7919147882$

C, $0,3.3105450464,1.7781026451,0.1605902016$

$\mathrm{H}, 0,3.4036325698,2.1220753789,-0.8756268621$

$\mathrm{H}, 0,3.0478723016,2.6434097437,0.7736441372$

C,0,4.6176125126,1.0851655358,0.6395324343

$\mathrm{H}, 0,4.979089604,1.4982639094,1.584258989$

$\mathrm{H}, 0,5.419640452,1.1951726418,-0.0967833155$

C,0,3.4880342991,-0.6576661843,-1.5868417197

$\mathrm{H}, 0,4.0187620232,-1.5931486314,-1.7840677792$

$\mathrm{H}, 0,2.6234868529,-0.6197445308,-2.2543702823$

$\mathrm{H}, 0,4.1596818523,0.1690730821,-1.8365810292$

$0,0,-5.3192543997,-0.3120814732,-0.5326100101$

$0,0,5.3397326718,-1.2930170165,0.4132596997$

$\mathrm{H}, 0,2.6577494279,-2.7544834537,0.3605886975$

$\mathrm{H}, 0,-3.8246181772,1.681059719,-1.0391248859$

$\mathrm{H}, 0,-3.1137564649,0.6424265374,-2.2868167197$

$0,0,-2.5423272692,1.3346013923,1.3025076398$

$\mathrm{H}, 0,-3.0052236091,2.1819460182,1.20236926$

$\mathrm{H}, 0,3.9925693653,-0.6409165766,1.8278708968$

$\mathrm{H}, 0,6.0393167977,-1.2264922736,1.077151676$ 
$\mathrm{H}, 0,-3.2135192564,0.7141738419,1.6888013518$ O,0,-4.410186594,-0.3464274013,2.3375723596 $\mathrm{H}, 0,-5.1286912777,-0.4248594197,1.69185641$ $\mathrm{H}, 0,-4.814215539,0.0389968521,3.1272661723$

common int1(SO) - complex with two $\mathrm{H}_{2} \mathrm{O}$ at $\mathrm{C}_{4}$ Charge $=1$ Multiplicity $=1$

C, $0,-0.8560604075,2.5265428043,0.2226892139$

C, $0,-1.6951712359,1.4007479056,-0.2820244456$

C, $0,-1.1758360117,0.1678220785,-0.6285606685$

C, $0,0.2210760575,-0.0579570699,-0.4636265492$

C, $0,1.1423458801,1.0780681115,-0.086406368$

C, $0,0.4411636457,2.0531718349,0.8532026109$

$\mathrm{H}, 0,-0.6405436531,3.1613173307,-0.6532574309$

$\mathrm{H}, 0,-1.4484078786,3.1383982348,0.9084625619$

$\mathrm{H}, 0,1.345617792,1.6134427791,-1.0317512477$ $\mathrm{H}, 0,0.2344031313,1.5533285897,1.8078707217$ $\mathrm{H}, 0,1.0986193974,2.902953165,1.0540061605$ C, $0,-2.0710538616,-0.9408283512,-1.1503872982$ C, $0,-3.1542885704,1.7079789128,-0.3819678819$ C, $0,-4.0311679519,0.5745467794,-0.8413854526$ C, $0,-3.3784675365,-0.4148331753,-1.7526315018$ $\mathrm{H}, 0,-1.5515710101,-1.4983308902,-1.9317032518$ $\mathrm{H}, 0,-4.0676138287,-1.2301912604,-1.9795463618$ $\mathrm{H}, 0,-2.2910611015,-1.6498920419,-0.3427576987$ $\mathrm{H}, 0,-3.1505150839,0.1253527523,-2.6817984451$ $\mathrm{H}, 0,-3.5121334461,2.0936967848,0.5790835423$ C, $0,2.4402839538,0.530752212,0.4803734864$ $\mathrm{H}, 0,2.1769790972,-0.0067546813,1.405211516$ C, $0,0.7598804377,-1.3669432833,-0.6807747826$ $\mathrm{H}, 0,0.0858757938,-2.1885818034,-0.8895258979$ C, $0,2.1034935635,-1.6041923232,-0.6691643471$ $\mathrm{H}, 0,2.467093825,-2.6073101163,-0.8900710474$ C, $0,3.0804776288,-0.5091899215,-0.4455612205$ C, $0,4.3410016902,-0.8492223716,0.3649456374$ $\mathrm{H}, 0,4.0493016659,-1.4729075305,1.2194486571$ C, $0,3.5929131132,1.4817791638,0.8140609341$ $\mathrm{H}, 0,3.7041818742,2.2405972699,0.0318477454$ $\mathrm{H}, 0,3.4317682004,2.0022796965,1.7605294138$ C, $0,4.8282788501,0.5378973028,0.8579411985$ $\mathrm{H}, 0,5.2520111455,0.4626437835,1.8618807468$ $\mathrm{H}, 0,5.620018405,0.8894818684,0.189719356$ C, $0,3.4763247523,0.0376236279,-1.8367925994$ $\mathrm{H}, 0,3.9149435018,-0.7611119191,-2.4397188829$ $\mathrm{H}, 0,2.6064301787,0.4252989082,-2.3724321054$ $\mathrm{H}, 0,4.2163234585,0.837147225,-1.7413431283$ $0,0,5.2859232758,-1.5539870807,-0.4257519217$ $\mathrm{H}, 0,5.9999630044,-1.8575399209,0.1510014692$ $0,0,-5.2037147384,0.5080374263,-0.4998480556$ H, $0,-3.2748350995,2.5360377001,-1.1001499342$ 
$0,0,-3.1700825322,-0.5185798865,2.043557363$

$\mathrm{H}, 0,-3.7184099398,-1.1440742076,1.5312681392$ $\mathrm{H}, 0,-2.2548542196,-0.7506191502,1.8402897497$ O,0,-4.9620763324,-2.1699888757,0.5883826639 $\mathrm{H}, 0,-5.6238033766,-2.5475381755,1.1829618418$ $\mathrm{H}, 0,-5.416053504,-1.4506592118,0.1206067955$

TS2 common(S0) - complex

Charge $=1$ Multiplicity $=1$

C,0,-0.9248661901,2.1692721772,-0.6847457306

C, $0,-1.7147718584,0.9170747259,-0.5152078509$

C, $0,-1.0994061304,-0.3224690648,-0.196183673$

C, $0,0.2854294851,-0.3961655113,-0.1103179873$

C, $0,1.1479804939,0.8275957672,-0.3805890044$

C, $0,0.4220603913,2.1079934798,0.0133384341$

$\mathrm{H}, 0,-0.7821530582,2.2873053695,-1.7717688597$

$\mathrm{H}, 0,-1.5303543799,3.0214541743,-0.3622837776$

$\mathrm{H}, 0,1.3159605239,0.8558442516,-1.4706128755$

$\mathrm{H}, 0,0.282524833,2.1267169031,1.1017443218$

$\mathrm{H}, 0,1.027816468,2.9756207858,-0.2616175382$

C, $0,-2.0377311457,-1.4898872855,0.0368694196$

C,0,-3.1347554992,1.0047771741,-0.6235303681

C, $0,-3.9017228413,-0.1945691894,-1.0288052067$

C,0,-3.1713319928,-1.505577432,-0.9904751734

$\mathrm{H}, 0,-1.5175982639,-2.4442416631,-0.0095846791$

$\mathrm{H}, 0,-3.8858377247,-2.3082178508,-0.7934265791$

$\mathrm{H}, 0,-2.4669759649,-1.4047445294,1.0445089109$

$\mathrm{H}, 0,-2.7613681965,-1.6601132083,-1.9983473439$

$\mathrm{H}, 0,-3.4650802032,1.0317625727,0.6417779003$

C,0,2.483580559,0.6711777092,0.3272928254

$\mathrm{H}, 0,2.2615931686,0.5880575098,1.4031809228$

C,0,0.949711846,-1.6468239188,0.2209394275

$\mathrm{H}, 0,0.3426970365,-2.5177613422,0.4343210547$

C,0,2.2965735909,-1.7748330951,0.260920529

$\mathrm{H}, 0,2.7339500525,-2.7480859964,0.4810384474$

C,0,3.1977972279,-0.629895195,-0.0580739798

C, $0,4.4696095559,-0.4857375321,0.7908294824$

$\mathrm{H}, 0,4.2121253627,-0.676818385,1.8407778069$

C, $0,3.5647120839,1.7443934461,0.1689754915$

$\mathrm{H}, 0,3.628350251,2.0770396736,-0.8730925286$

$\mathrm{H}, 0,3.3624981453,2.6238784377,0.7845310633$

$\mathrm{C}, 0,4.861267725,1.0024908185,0.6003066362$

$\mathrm{H}, 0,5.2834968803,1.4125305072,1.5207457591$

$\mathrm{H}, 0,5.6322100724,1.0665062711,-0.1736250963$

C,0,3.5699412257,-0.7241978422,-1.5526470865

$\mathrm{H}, 0,4.056578184,-1.6821712555,-1.7544617709$

$\mathrm{H}, 0,2.6798266916,-0.6628493951,-2.1838680062$

$\mathrm{H}, 0,4.260787785,0.0730971882,-1.8419148531$

$\mathrm{O}, 0,5.4727478146,-1.40685369,0.3849879283$

$\mathrm{H}, 0,6.2019707566,-1.358066075,1.0178208675$ 
$0,0,-5.0848694172,-0.1089857771,-1.3525846556$

$\mathrm{H}, 0,-3.5403459858,1.948504038,-0.9897179151$

$\mathrm{O}, 0,-3.9656876849,1.0043164071,1.9035469254$

$\mathrm{H}, 0,-4.6470981232,0.2763961118,1.8652329058$

$\mathrm{H}, 0,-3.2560785669,0.6932444735,2.4881590799$

O,0,-5.7401004633,-0.9455226435,1.4997774169

$\mathrm{H}, 0,-6.5890613826,-0.8415560942,1.9515184648$

$\mathrm{H}, 0,-5.9382961376,-0.861762001,0.5539605182$

trenbolone(SO) - complex with $\mathrm{H}_{3} \mathrm{O}^{+}$and $\mathrm{H}_{2} \mathrm{O}$ at $\mathrm{C}_{3}$ carbonyl

Charge $=1$ Multiplicity $=1$

C,0,-0.4219517666,2.6784445585,-0.6575812992

C, $0,-1.3478492284,1.5142146576,-0.4644466769$

$\mathrm{C}, 0,-0.8266732892,0.2219950575,-0.0753854508$

C, $0,0.5288952363,0.0368358185,0.0539090736$

C, $0,1.5089497488,1.1724485093,-0.2272821802$

C, $0,0.8803287236,2.5201368539,0.1076536008$

$\mathrm{H}, 0,-0.2045980622,2.72961137,-1.7354044569$

$\mathrm{H}, 0,-0.9480174871,3.6022015631,-0.4012675619$

$\mathrm{H}, 0,1.7291885385,1.1632931724,-1.307084264$

$\mathrm{H}, 0,0.6960173626,2.5766816564,1.1883125814$

$\mathrm{H}, 0,1.5681931258,3.3286023196,-0.1566112795$

C,0,-1.8604729729,-0.8340876575,0.2568211192

$\mathrm{C}, 0,-2.6964118178,1.7162569912,-0.69030765$

C,0,-3.5844619101,0.6319443559,-0.7952363515

C, $0,-3.0868771103,-0.7659257998,-0.6503291906$

$\mathrm{H}, 0,-1.4504022021,-1.8401504148,0.1911120847$

$\mathrm{H}, 0,-3.8855265703,-1.4099781387,-0.2728347782$

$\mathrm{H}, 0,-2.1857882258,-0.6825843107,1.2945431944$

$\mathrm{H}, 0,-2.843607476,-1.1089250227,-1.66653261$

$\mathrm{H}, 0,-5.3800344133,-2.3779627849,1.3309913205$

C, $0,2.7974493749,0.927504369,0.5413176374$

$\mathrm{H}, 0,2.5278514745,0.9037017393,1.609431426$

$\mathrm{C}, 0,1.0811391716,-1.252401244,0.4777463845$

$\mathrm{H}, 0,0.3919018031,-2.0527501125,0.7200210374$

C,0,2.4048266225,-1.4960789398,0.5740245018

$\mathrm{H}, 0,2.7503662001,-2.4880915943,0.8623905041$

C, $0,3.4123415369,-0.4446315719,0.2331439286$

C,0,4.6615692077,-0.36862616,1.1226604038

$\mathrm{H}, 0,4.3526998834,-0.4935079708,2.1690747375$

C, $0,3.9711946955,1.9013801015,0.3917833067$

$\mathrm{H}, 0,4.0976416786,2.1951819674,-0.6564682085$

$\mathrm{H}, 0,3.8230090114,2.81415958,0.9736945471$

C, $0,5.1869552206,1.0706608274,0.8902723907$

$\mathrm{H}, 0,5.6186297401,1.4797492868,1.8069482922$

$\mathrm{H}, 0,5.9814301081,1.0376012354,0.1383089369$

C,0,3.8256272336,-0.6243256186,-1.2412946879

$\mathrm{H}, 0,4.248211723,-1.621784691,-1.3907367375$

$\mathrm{H}, 0,2.9615664947,-0.5265341668,-1.9037509095$

$\mathrm{H}, 0,4.5798246885,0.109710254,-1.5410606782$ 
O,0,5.598404195,-1.387106484,0.7938266616

$\mathrm{H}, 0,6.3221204677,-1.3493330221,1.4336687316$

$0,0,-4.8128903898,0.8647202726,-1.0978458145$

$\mathrm{H}, 0,-3.0749546308,2.7112978734,-0.9022335083$

$0,0,-6.0163720403,-2.7653117901,0.7137396306$

$\mathrm{H}, 0,-6.2100031266,-1.7277058748,-0.6126511818$

$\mathrm{H}, 0,-5.6146255738,-3.59746089,0.4278667079$

$\mathrm{O}, 0,-6.2723674295,-1.1062235387,-1.3830123542$

$\mathrm{H}, 0,-7.1976085391,-0.8279131536,-1.4429327856$

$\mathrm{H}, 0,-5.4261090049,0.0022195615,-1.1974471253$

\section{Structures for alkaline reversion}

\section{$17 \alpha$}

cis- $12 \mathrm{OH}(\mathrm{SO})$ - complex with $\mathrm{HO}^{-}$and $\mathrm{H}_{2} \mathrm{O}$ at $\mathrm{C}_{3}$ carbonyl and $\mathrm{H}_{2} \mathrm{O}$ and $\mathrm{C}_{12}$

Charge $=-1$ Multiplicity $=1$

C,0,-1.6272147223,-2.0031709688,-0.7246381762

C, $0,-2.1407890892,-0.6123072563,-0.4500092609$

C, $0,-1.3327167512,0.4314155531,-0.1654129664$

C, $0,0.138233488,0.2533146464,-0.1062806459$

C, $0,0.6573260377,-1.1752966224,-0.0952015013$

$\mathrm{C}, 0,-0.1461712027,-2.0253717581,-1.0790899065$

$\mathrm{H}, 0,-1.8122856621,-2.6224932392,0.1655634668$

$\mathrm{H}, 0,-2.2214824965,-2.4487917742,-1.5308349043$

$\mathrm{H}, 0,0.4887313533,-1.5873353891,0.9140888022$

$\mathrm{H}, 0,-0.0015818503,-1.6253967323,-2.0918241463$

$\mathrm{H}, 0,0.2279031088,-3.0548862443,-1.07735366$

C,0,-1.8938609123,1.813043368,0.1042537382

C,0,-3.6430919421,-0.4865689291,-0.5399507421

C,0,-4.1659294798,0.9107592518,-0.3554159345

C,0,-3.3599340927,1.7803898898,0.5578169793

$\mathrm{H}, 0,-1.3091164765,2.3011499273,0.8888312826$

$\mathrm{H}, 0,-3.7953188324,2.7806299667,0.6032973729$

$\mathrm{H}, 0,-1.7956817083,2.4407665316,-0.7906358153$

$\mathrm{H}, 0,-3.410958053,1.3230201679,1.5553855623$

$\mathrm{H}, 0,-4.1100420766,-1.1045102623,0.2442326845$

C, $0,2.1480606661,-1.1947638178,-0.3978163173$

$\mathrm{H}, 0,2.2680496957,-0.8924230442,-1.4493535159$

C, $0,0.9797664132,1.303434444,-0.0631584594$

$\mathrm{H}, 0,0.5913497725,2.3205805384,-0.0892495575$

C, $0,2.4881089601,1.2158875413,0.0037392135$

$\mathrm{H}, 0,2.9037143791,1.4213476845,-0.993364128$

C,0,2.9325173662,-0.1738150783,0.4505253049

C, $0,4.3853200369,-0.5764286121,0.1411898283$

C, $0,2.8958861265,-2.522772376,-0.185319425$

$\mathrm{H}, 0,2.4326735613,-3.0949814429,0.626473971$

$\mathrm{H}, 0,2.8638213549,-3.1498804692,-1.0794313738$

C,0,4.3462779544,-2.114607337,0.1905343786 
$\mathrm{H}, 0,5.0950049906,-2.5304801753,-0.4905575663$ $\mathrm{H}, 0,4.6030072304,-2.4572607878,1.1966614115$ C, $0,2.6879312538,-0.3658370219,1.9548956193$ $\mathrm{H}, 0,3.4289351125,0.1892221559,2.5370825811$ $\mathrm{H}, 0,1.6966052148,-0.0052299623,2.2426621763$ $\mathrm{H}, 0,2.7578397062,-1.4198792485,2.238987049$ $\mathrm{O}, 0,-5.1871320596,1.2938591382,-0.9145783555$ $\mathrm{H}, 0,-4.0081690131,-0.8801698234,-1.4956091799$ $\mathrm{O}, 0,3.0025293508,2.2055999181,0.9124856641$ $\mathrm{H}, 0,2.64096244,3.0675713045,0.6583566194$ $\mathrm{H}, 0,4.7596297179,2.5393663572,0.2098033582$ $\mathrm{O}, 0,5.531284402,2.4771724165,-0.3812212613$ $\mathrm{H}, 0,5.3851485344,1.6241174009,-0.8296679$ $\mathrm{H}, 0,5.096007148,-0.1490496364,0.8597677317$ $\mathrm{O}, 0,4.7307524714,-0.121263513,-1.1768164818$ $\mathrm{H}, 0,5.532429033,-0.5872057901,-1.4542181583$ $0,0,-6.0668827031,-0.0721730634,2.1324412136$ $\mathrm{H}, 0,-6.5918456997,-0.3881555391,-0.7128156523$ $0,0,-7.0189549234,-0.9047986248,-0.0161107421$ $\mathrm{H}, 0,-6.600338098,-0.5315846535,0.8853143487$ $\mathrm{H}, 0,-5.8109770357,0.8514019909,2.0147373764$

TS1 cis-12OH(SO) - complex

Charge $=-1$ Multiplicity $=1$

C,0,-1.7715559771,-1.8429203572,-0.7328586122 $\mathrm{C}, 0,-2.2478024868,-0.4247850464,-0.5306382382$ $\mathrm{C}, 0,-1.3955863128,0.6013126959,-0.2863468431$ C, $0,0.0611076944,0.3793235217,-0.1842362865$ C, $0,0.5270376935,-1.0662361623,-0.0960584858$ C,0,-0.2868465072,-1.9288562038,-1.060454965 $\mathrm{H}, 0,-1.989423312,-2.4152741317,0.1812505799$ $\mathrm{H}, 0,-2.3696929795,-2.3066959701,-1.5262636183$ $\mathrm{H}, 0,0.3195024983,-1.4270306421,0.9256576733$ $\mathrm{H}, 0,-0.1108416585,-1.5745813177,-2.085393903$ $\mathrm{H}, 0,0.0533523955,-2.9691664681,-1.011063099$ C, $0,-1.9315346398,2.0125135753,-0.14542931$ C, $0,-3.7242264428,-0.2561369899,-0.5915733618$ C, $0,-4.2580582435,1.079529004,-0.4302135781$ C, $0,-3.385144551,2.0412346335,0.3355801885$ $\mathrm{H}, 0,-1.3260179002,2.5765847398,0.5697872276$ $\mathrm{H}, 0,-3.811335544,3.0459521981,0.2832163361$ $\mathrm{H}, 0,-1.8450094402,2.5429204761,-1.1044893798$ $\mathrm{H}, 0,-3.4210379238,1.7116358469,1.3845166555$ $\mathrm{H}, 0,-4.1832432123,-0.8270703859,0.4765795256$ C,0,2.0227538185,-1.156227911,-0.3580566926 $\mathrm{H}, 0,2.1822060639,-0.9045272366,-1.4176062507$ $\mathrm{C}, 0,0.9459998346,1.3972828092,-0.1674968243$ $\mathrm{H}, 0,0.5995599897,2.4261320842,-0.2557958957$ C, $0,2.4461362277,1.2547600409,-0.0484753415$ $\mathrm{H}, 0,2.9060486488,1.4042146241,-1.0363024327$ 
C, $0,2.8223067685,-0.130489521,0.4695374144$ $\mathrm{C}, 0,4.2665846902,-0.6021817495,0.2256505111$ C, $0,2.7123399102,-2.5014548072,-0.0683924557$ $\mathrm{H}, 0,2.1999924357,-3.0239995954,0.7473687469$ $\mathrm{H}, 0,2.6878205484,-3.1619950861,-0.9384571852$ C, $0,4.163723637,-2.1334256156,0.3440136902$ $\mathrm{H}, 0,4.9194889939,-2.6105590436,-0.2873643589$ $\mathrm{H}, 0,4.3682908285,-2.4368316315,1.3744807636$ C, $0,2.5253698294,-0.2497946125,1.9721418606$ $\mathrm{H}, 0,3.2793372268,0.2860432094,2.5559917468$ $\mathrm{H}, 0,1.5477711655,0.1746749554,2.2159723586$ $\mathrm{H}, 0,2.5283459569,-1.2940566331,2.297834317$ $0,0,-5.4082527461,1.4080793362,-0.7829643362$ $\mathrm{H}, 0,-4.2114317805,-0.8280370944,-1.3874855468$ $\mathrm{O}, 0,2.9715037878,2.2606321262,0.8387863018$ $\mathrm{H}, 0,2.635010056,3.1214548228,0.5500582786$ $\mathrm{H}, 0,4.7637227004,2.4967045657,0.1706166152$ $0,0,5.5474335563,2.3732953647,-0.3942714741$ $\mathrm{H}, 0,5.3787312155,1.5043835573,-0.80339418$ $\mathrm{H}, 0,4.9716953747,-0.1691253849,0.9463879867$ $0,0,4.6703090417,-0.2231871491,-1.100087986$ $\mathrm{H}, 0,5.4645547204,-0.7272820621,-1.3277726206$ O,0,-4.7146769291,-1.4674518251,1.5628248989 $\mathrm{H}, 0,-6.6384934616,-0.0743042339,-0.2107532251$ $0,0,-6.9626326832,-0.8076086395,0.3370778573$ $\mathrm{H}, 0,-6.1561220142,-1.0783791504,0.8652927427$ H, $0,-4.5356675625,-0.8833705297,2.3123802096$

enolate cis- $12 \mathrm{OH}(\mathrm{SO})$ - complex with three $\mathrm{H}_{2} \mathrm{O}$ Charge $=-1$ Multiplicity $=1$

C,0,-1.78358,-1.818431,-0.921855

$\mathrm{C}, 0,-2.265494,-0.3965,-0.743639$

C, $0,-1.407613,0.625643,-0.448251$

$\mathrm{C}, 0,0.033217,0.402365,-0.280668$

$\mathrm{C}, 0,0.489798,-1.048526,-0.190053$

C, $0,-0.287366,-1.901863,-1.192549$

$\mathrm{H}, 0,-2.029452,-2.388804,-0.013555$

$\mathrm{H}, 0,-2.351437,-2.286168,-1.734565$

$\mathrm{H}, 0,0.242321,-1.419182,0.819099$

$\mathrm{H}, 0,-0.071287,-1.539294,-2.207071$

$\mathrm{H}, 0,0.051078,-2.94276,-1.138751$

C, $0,-1.981183,2.02695,-0.399431$

C, $0,-3.692929,-0.179555,-0.90464$

C, $0,-4.302967,0.996852,-0.510287$

C,0,-3.414504,2.045291,0.131419

$\mathrm{H}, 0,-1.367438,2.680952,0.225995$

$\mathrm{H}, 0,-3.868447,3.031565,-0.00373$

$\mathrm{H}, 0,-1.96627,2.461867,-1.412294$

$\mathrm{H}, 0,-3.413473,1.839475,1.214337$

$\mathrm{H}, 0,-3.93966,-0.824297,1.14975$ 
C,0,1.993934,-1.145864,-0.394844

$\mathrm{H}, 0,2.197023,-0.878227,-1.442977$

C, $0,0.930308,1.412764,-0.211058$

$\mathrm{H}, 0,0.59771,2.445658,-0.304853$

C, $0,2.422005,1.255782,-0.028874$

$\mathrm{H}, 0,2.929144,1.419549,-0.991223$

C, $0,2.767659,-0.140712,0.480539$

$\mathrm{C}, 0,4.217034,-0.620888,0.286993$

C, $0,2.661285,-2.501684,-0.101339$

$\mathrm{H}, 0,2.112144,-3.034302,0.683526$

$\mathrm{H}, 0,2.667204,-3.146792,-0.983302$

C,0,4.097461,-2.152994,0.375686

$\mathrm{H}, 0,4.874293,-2.625992,-0.232941$

$\mathrm{H}, 0,4.258255,-2.475601,1.408112$

C,0,2.40987,-0.282392,1.968113

$\mathrm{H}, 0,3.143626,0.237882,2.590517$

$\mathrm{H}, 0,1.426543,0.146587,2.178868$

$\mathrm{H}, 0,2.391437,-1.331791,2.276517$

$\mathrm{O}, 0,-5.577694,1.227595,-0.559351$

$\mathrm{H}, 0,-4.302761,-0.989352,-1.301811$

$\mathrm{O}, 0,2.919423,2.242042,0.898508$

$\mathrm{H}, 0,2.589627,3.108352,0.619081$

$\mathrm{H}, 0,4.731867,2.47238,0.308307$

$0,0,5.538221,2.353843,-0.225395$

$\mathrm{H}, 0,5.380076,1.495248,-0.659665$

$\mathrm{H}, 0,4.896326,-0.205633,1.042155$

$0,0,4.677088,-0.224772,-1.015432$

$\mathrm{H}, 0,5.475611,-0.732441,-1.218514$

$0,0,-4.20822,-1.210082,2.005438$

$\mathrm{H}, 0,-6.387861,-0.143617,0.113739$

$0,0,-6.771989,-0.910501,0.60554$

$\mathrm{H}, 0,-6.059304,-1.174486,1.209974$

$\mathrm{H}, 0,-4.143145,-0.483198,2.639234$

TS2 cis- $12 \mathrm{OH}(\mathrm{SO})$ - complex
Charge $=-1$ Multiplicity $=1$
C, $0,-1.6832155963,-2.3716685159,-0.2933636718$
C, $0,-2.2832203756,-1.0310252118,-0.6416975747$
C, $0,-1.4560842787,0.1192276913,-0.7398736196$
C, $0,-0.0811876834,0.0562826367,-0.4817425545$
C, $0,0.5190338548,-1.2509251556,0.0361950241$
C, $0,-0.1958797799,-2.440381986,-0.6006389882$
$\mathrm{H}, 0,-1.8370585908,-2.5324295663,0.7844363968$
$\mathrm{H}, 0,-2.2420111752,-3.1597254485,-0.8077325757$
$\mathrm{H}, 0,0.3419205627,-1.3058714018,1.1221662112$
$\mathrm{H}, 0,-0.0318229668,-2.4224720026,-1.6863946553$
$\mathrm{H}, 0,0.2264046725,-3.3754042981,-0.2175019925$
C,0,-2.119254244,1.3838518217,-1.2441329652
C, $0,-3.6623155439,-0.9723393627,-0.8386141835$
C,0,-4.3624967614,0.2659418449,-0.9079434787 
C, $0,-3.5489914543,1.5252836157,-0.7261079647$ $\mathrm{H}, 0,-1.5529410177,2.2689326745,-0.9479464221$ $\mathrm{H}, 0,-4.0693980392,2.3591035067,-1.2045420112$ $\mathrm{H}, 0,-2.1380085149,1.371622999,-2.3449350155$ $\mathrm{H}, 0,-3.5363467528,1.7274240341,0.3563835278$ $\mathrm{H}, 0,-3.5637186588,-0.7179972338,1.5168241824$ C, $0,2.0216686909,-1.2702987799,-0.2073156101$ $\mathrm{H}, 0,2.1846696023,-1.2620802199,-1.29457876$ C, $0,0.796374438,1.1252524539,-0.8041825437$ $\mathrm{H}, 0,0.4179535491,1.9402318017,-1.4135130654$ C, $0,2.149752275,1.1477559978,-0.4708724944$ $\mathrm{H}, 0,2.8282755633,1.6712310584,-1.1344713629$ C, $0,2.6842447988,0.0082330841,0.360549559$ C, $0,4.1781987828,-0.3224349506,0.2298908843$ C, $0,2.8421687572,-2.4206396697,0.3876858497$ $\mathrm{H}, 0,2.415005757,-2.7472564704,1.3423652764$ $\mathrm{H}, 0,2.8553877088,-3.2902332874,-0.2739551893$ C, $0,4.2602177643,-1.8211580064,0.5954753602$ $\mathrm{H}, 0,5.0147972196,-2.3085639452,-0.029381872$ $\mathrm{H}, 0,4.5841097641,-1.9320274553,1.6341136628$ C, $0,2.3677462609,0.17165487,1.8579527507$ $\mathrm{H}, 0,3.043243428,0.9033648502,2.3051868739$ $\mathrm{H}, 0,1.3449233196,0.521807927,2.0145099753$ $\mathrm{H}, 0,2.4903311483,-0.7770425992,2.3898105346$ $0,0,-5.6142163773,0.3418870278,-1.0139845219$ $\mathrm{H}, 0,-4.2472390552,-1.8889701316,-0.8484097818$ $\mathrm{O}, 0,2.5372755459,2.7895164445,0.7711021655$ $\mathrm{H}, 0,2.0670113943,3.4277593507,0.2176099557$ $\mathrm{H}, 0,4.0040048011,2.8130488619,0.2333513063$ $0,0,4.9507807005,2.8053218475,-0.1475030292$ $\mathrm{H}, 0,5.0186119517,1.9711063157,-0.6364122668$ $\mathrm{H}, 0,4.7898876268,0.2935239819,0.8999553267$ $0,0,4.5858281604,-0.0740312672,-1.1210208643$ $\mathrm{H}, 0,5.4693332094,-0.4477606513,-1.244816317$ $0,0,-3.6203116366,-0.2530099483,2.3675224723$ $\mathrm{H}, 0,-6.132534269,0.9464395634,0.8111438482$ O,0,-6.1030878417,1.0706258989,1.7743343704 $\mathrm{H}, 0,-5.2671351408,0.6458791532,2.0426116825$ $\mathrm{H}, 0,-2.9446805543,0.4371392522,2.3076161549$

trans-5OH(SO) - complex with $\mathrm{HO}^{-}$and $\mathrm{H}_{2} \mathrm{O}$ at $\mathrm{C}_{4}$ and $\mathrm{H}_{2} \mathrm{O}$ and $\mathrm{C}_{5}$

Charge $=-1$ Multiplicity $=1$

C, $0,0.8453832669,-0.704485739,-2.1287000917$

C, $0,1.7021793694,-0.7551282461,-0.8649052175$

C, $0,0.9646730449,-0.317064957,0.3945774634$

C, $0,-0.3471837806,0.0181169673,0.3820377337$

C, $0,-1.2065252173,-0.1369185176,-0.8743374792$

C, $0,-0.5854161684,-1.1321601463,-1.8466100661$

$\mathrm{H}, 0,0.8386739779,0.3250189693,-2.5079288643$

$\mathrm{H}, 0,1.3248965527,-1.3286111858,-2.8917774469$ 
$\mathrm{H}, 0,-1.2531721413,0.8386140523,-1.3845291844$

$\mathrm{H}, 0,-0.6083135585,-2.1412064791,-1.4124641521$

$\mathrm{H}, 0,-1.1660168234,-1.1587720157,-2.7743755099$

C,0,1.8455340062,-0.3224998293,1.6224099589

C,0,2.9608219223,0.1125959509,-1.0751676631

C,0,3.8257227767,0.1699730019,0.1539619704

C,0,3.1203007244,0.5226837408,1.4332102491

$\mathrm{H}, 0,1.3232974922,0.0318422311,2.5089732809$

$\mathrm{H}, 0,3.8090867515,0.403687596,2.2727265367$

$\mathrm{H}, 0,2.1410740454,-1.3579087833,1.8302192767$

$\mathrm{H}, 0,2.8362689977,1.581484535,1.3554515697$

$\mathrm{H}, 0,2.6369348374,1.138258862,-1.2958449222$

$\mathrm{C}, 0,-2.6114547211,-0.5261401647,-0.4352869127$

$\mathrm{H}, 0,-2.509781217,-1.4326693867,0.1809218965$

$\mathrm{C}, 0,-1.0344199373,0.5353110697,1.5867783955$

$\mathrm{H}, 0,-0.4308075157,0.7690518825,2.4573599333$

C,0,-2.3578541554,0.7642328339,1.6575403472

$\mathrm{H}, 0,-2.7853700156,1.1861603636,2.5663325107$

C,0,-3.2491818603,0.5366030096,0.4745858948

C,0,-4.6302971391,-0.0852260566,0.7277418651

C,0,-3.6992128782,-0.8044751464,-1.4740750004

$\mathrm{H}, 0,-3.6637769473,-0.0609412665,-2.278782289$

$\mathrm{H}, 0,-3.5937930223,-1.7903255758,-1.9338392471$

C, $0,-5.0156754122,-0.6741827823,-0.654300621$

$\mathrm{H}, 0,-5.5003318629,-1.6440899099,-0.505279728$

$\mathrm{H}, 0,-5.7379862265,-0.0271085485,-1.1592999618$

C, $0,-3.4756625148,1.894282757,-0.2222290354$

$\mathrm{H}, 0,-3.9296039322,2.5934318106,0.4880259922$

$\mathrm{H}, 0,-2.5332765772,2.3315656376,-0.5618906116$

$\mathrm{H}, 0,-4.1483623988,1.8075441336,-1.081439897$

$\mathrm{O}, 0,5.0297926515,-0.0577795122,0.1091592053$

$\mathrm{H}, 0,3.5384868615,-0.2669804814,-1.9242957903$

$\mathrm{H}, 0,-5.3559361954,0.6588652278,1.0786446106$

$0,0,-4.4946070269,-1.1054625686,1.7212748171$

$\mathrm{H}, 0,-5.3389893197,-1.571061229,1.7911523207$

$\mathrm{O}, 0,1.0034873037,3.0611450395,-0.3973370359$

$\mathrm{H}, 0,3.9143743912,3.0115447494,-0.8361519924$

$\mathrm{O}, 0,3.4074832871,3.6704452923,-0.3461651993$

$\mathrm{H}, 0,2.3869621116,3.3565078875,-0.3732640494$

$\mathrm{H}, 0,0.8695605314,2.14841505,-0.1091018473$

$\mathrm{O}, 0,2.1234961702,-2.1159703639,-0.613622893$

$\mathrm{H}, 0,2.5534477416,-2.4479824585,-1.4174648223$

$\mathrm{H}, 0,3.4734329371,-2.6630226515,0.62058826$

$\mathrm{O}, 0,4.1988150328,-2.9839141231,1.1873391562$

$\mathrm{H}, 0,4.8418722436,-2.2621581741,1.1955343266$

TS1 trans-5OH(SO) - complex

Charge $=-1$ Multiplicity $=1$

C, $0,-0.7739233785,0.0097204857,2.1673659958$

C,0,-1.5725117001,-0.5956018757,1.0100979507 
C, $0,-0.8142606018,-0.6050242751,-0.3111079604$

C, $0,0.4600484736,-0.1643475605,-0.4224879836$

C, $0,1.273127298,0.2621258448,0.8024352982$

C, $0,0.7015631362,-0.345714097,2.0783206365$

$\mathrm{H}, 0,-0.876227263,1.1004316514,2.1345369611$

$\mathrm{H}, 0,-1.2258804281,-0.3266952758,3.1084594577$

$\mathrm{H}, 0,1.2094986823,1.3578261853,0.9013367962$

$\mathrm{H}, 0,0.8373523171,-1.4360285633,2.0618896871$

$\mathrm{H}, 0,1.2422722672,0.0401017194,2.9489925171$

C,0,-1.6517824338,-1.1541746131,-1.4410677693

C,0,-2.8905432073,0.1510959117, 0.8463907723

$\mathrm{C}, 0,-3.6791102761,-0.0734118025,-0.3358232488$

C, $0,-2.9437007582,-0.3498393765,-1.6295574808$

$\mathrm{H}, 0,-1.1080809952,-1.1929485031,-2.3838352062$

$\mathrm{H}, 0,-3.6281910252,-0.8466789422,-2.322750767$

$\mathrm{H}, 0,-1.9191864063,-2.1890053477,-1.1934952931$

$\mathrm{H}, 0,-2.7043771736,0.6368932882,-2.0538330176$

$\mathrm{H}, 0,-2.5365426002,1.4033867551,0.6004022865$

C, $0,2.7264623588,-0.1214225699,0.5596050474$

$\mathrm{H}, 0,2.736051659,-1.2021664276,0.3500807154$

C, $0,1.1558062059,-0.1043039283,-1.727538627$

$\mathrm{H}, 0,0.5748891832,-0.304646151,-2.6218863534$

C, $0,2.456145973,0.2063545332,-1.875626296$

$\mathrm{H}, 0,2.8887993697,0.2659755529,-2.8736067029$

C, $0,3.30713212,0.5576199238,-0.6922351177$

C, $0,4.7497117243,0.0310116625,-0.6680820581$

C, $0,3.7859798166,0.1407706874,1.6314478601$

$\mathrm{H}, 0,3.6461817151,1.1317047577,2.0790626497$

$\mathrm{H}, 0,3.7488320021,-0.5921561889,2.4413515078$

C, $0,5.1223289917,0.0741148521,0.8367779276$

$\mathrm{H}, 0,5.7012707638,-0.8206430469,1.0861202$

$\mathrm{H}, 0,5.757160191,0.9384409355,1.0501030697$

C, $0,3.3764158819,2.0945395791,-0.5855529052$

$\mathrm{H}, 0,3.8017167489,2.5030707137,-1.5084528601$

$\mathrm{H}, 0,2.3827076792,2.5305946041,-0.4560302988$

$\mathrm{H}, 0,4.009024421,2.4217040573,0.2459674728$

$\mathrm{O}, 0,-4.9200607803,0.1030951327,-0.3720278424$

$\mathrm{H}, 0,-3.4836312202,0.174126236,1.7670768704$

$\mathrm{H}, 0,5.4204729286,0.6473042162,-1.2795648529$

$0,0,4.7541187821,-1.3056508233,-1.1779863522$

$\mathrm{H}, 0,5.6381051775,-1.67447154,-1.0478940691$

$\mathrm{O}, 0,-2.1518826335,2.6509450736,0.3451093635$

$\mathrm{H}, 0,-4.7825573534,2.1750753915,-1.0145087417$

$0,0,-4.3211894692,3.0212421075,-1.1057461705$

$\mathrm{H}, 0,-3.4711766356,2.9021660407,-0.585667516$

$\mathrm{H}, 0,-1.3485221369,2.5390794914,-0.18305382$

$\mathrm{O}, 0,-1.8182058555,-2.0048040945,1.315505526$

$\mathrm{H}, 0,-2.2197678755,-2.0389067111,2.1976118815$

$\mathrm{H}, 0,-3.3748338926,-2.6919862275,0.4830365037$

$\mathrm{O}, 0,-4.2062454719,-3.0547831789,0.1205871172$

$\mathrm{H}, 0,-4.8174242954,-2.3060352697,0.0894232387$ 
enolate trans- $5 \mathrm{OH}(\mathrm{SO})$ - complex with three $\mathrm{H}_{2} \mathrm{O}$

Charge $=-1$ Multiplicity $=1$

C, $0,-0.7811851684,-0.1338619772,2.2485937662$

C, $0,-1.5723817573,-0.7603821297,1.095184543$

C, $0,-0.8345535631,-0.7031858622,-0.2346799278$

C, $0,0.4238035236,-0.2200021223,-0.350401793$

C, $0,1.2405993156,0.2031387757,0.8722482189$

C, $0,0.7047286478,-0.4436227744,2.1438180909$

$\mathrm{H}, 0,-0.9166866855,0.9545049711,2.2200262082$

$\mathrm{H}, 0,-1.2152423349,-0.4838384738,3.1932188897$

$\mathrm{H}, 0,1.1381950493,1.2936159228,0.9957094347$

$\mathrm{H}, 0,0.8722962736,-1.528977208,2.1108311218$

$\mathrm{H}, 0,1.2422270116,-0.0528223161,3.0144070002$

$\mathrm{C}, 0,-1.6991453684,-1.1840621772,-1.3718402496$

C, $0,-2.9233273905,-0.1035786868,0.967529962$

C,0,-3.5809967755,0.1039837642,-0.2129323032

C,0,-2.9263753835,-0.2795498817,-1.5285061054

$\mathrm{H}, 0,-1.1578845912,-1.2491460625,-2.315420417$

$\mathrm{H}, 0,-3.6836574544,-0.7620778226,-2.1571378506$

$\mathrm{H}, 0,-2.0387040683,-2.1996343727,-1.1357569198$

$\mathrm{H}, 0,-2.6419261377,0.652550423,-2.0390236041$

$\mathrm{H}, 0,-1.8845157761,1.866729447,0.2771790113$

C,0,2.703079936,-0.1126147595,0.5936192004

$\mathrm{H}, 0,2.7546527129,-1.1830824052,0.3422938574$

$\mathrm{C}, 0,1.0886392885,-0.0801508799,-1.6648324565$

$\mathrm{H}, 0,0.4995897336,-0.2766601134,-2.5550764815$

C,0,2.3719579741,0.291519179,-1.8243176148

$\mathrm{H}, 0,2.7839918407,0.404103965,-2.8264458557$

$\mathrm{C}, 0,3.2275263332,0.6392261049,-0.6425409209$

C, $0,4.6933264686,0.181274587,-0.6634553339$

C, $0,3.7711565071,0.1557088944,1.6553967636$

$\mathrm{H}, 0,3.5939522875,1.1185723774,2.1485883561$

$\mathrm{H}, 0,3.7848732642,-0.6116370321,2.4335666177$

C, $0,5.0930694233,0.1872737727,0.8346057195$

$\mathrm{H}, 0,5.7223681238,-0.6840427606,1.0407346697$

$\mathrm{H}, 0,5.686628364,1.0753855284,1.0685856403$

C,0,3.2260405932,2.1735006202,-0.4804551701

$\mathrm{H}, 0,3.6221075641,2.6333211622,-1.3921619388$

$\mathrm{H}, 0,2.2143095692,2.5586836722,-0.3279369859$

$\mathrm{H}, 0,3.8511650471,2.4999641728,0.3569763109$

$0,0,-4.7604129651,0.6755844412,-0.313715943$

$\mathrm{H}, 0,-3.4112526914,0.1906217419,1.8977953428$

$\mathrm{H}, 0,5.3228828458,0.8507774087,-1.2628091972$

$0,0,4.7488955969,-1.1338977632,-1.2254835699$

$\mathrm{H}, 0,5.6516381303,-1.4659444907,-1.1285989044$

$\mathrm{O}, 0,-1.4162669529,2.6177126694,-0.1333001676$

$\mathrm{H}, 0,-4.3914878579,2.2289078374,-0.9554034046$

$0,0,-4.0654760025,3.1101893187,-1.269581422$

$\mathrm{H}, 0,-3.1294827768,3.1164922147,-1.0090034741$ 
$\mathrm{H}, 0,-0.737687131,2.1965981989,-0.6814336524$

O,0,-1.7152489334,-2.1965856533,1.3868113159

$\mathrm{H}, 0,-2.120954863,-2.2683845794,2.2647606207$

$\mathrm{H}, 0,-3.3069872149,-2.8072585791,0.5596870253$

$\mathrm{O}, 0,-4.1515782046,-3.1643990872,0.2222546024$

$\mathrm{H}, 0,-4.6669513775,-2.3782322002,-0.0036526262$

TS2 trans-5OH(SO) - complex

Charge $=-1$ Multiplicity $=1$

C, $0,0.832901236,-1.2158119518,-1.8618200184$

C, $0,1.64869233,-0.8843159973,-0.6323129211$

C, $0,0.9421560878,-0.3643096222,0.5616886285$

C, $0,-0.3750619537,-0.0384961249,0.5043220953$

C,0,-1.1917535093,-0.2935919772,-0.7657793685

C,0,-0.628200955,-1.4828177917,-1.5391632919

$\mathrm{H}, 0,0.8875616057,-0.3385887912,-2.5226217259$

$\mathrm{H}, 0,1.307058989,-2.0398793361,-2.4034509267$

$\mathrm{H}, 0,-1.1078233197,0.5920593004,-1.4170597816$

$\mathrm{H}, 0,-0.7330335084,-2.3957338661,-0.939450209$

$\mathrm{H}, 0,-1.1946333605,-1.6243714328,-2.4653639227$

C, $0,1.8503418841,-0.2100513538,1.7533321348$

C, $0,2.9729176574,-0.460954159,-0.8465580795$

C, $0,3.6985129272,0.3076147013,0.0867121129$

$\mathrm{C}, 0,3.0497162564,0.6728816392,1.4044225121$

$\mathrm{H}, 0,1.3384584535,0.1905667777,2.6275077855$

$\mathrm{H}, 0,3.8154150054,0.627341403,2.1849991121$

$\mathrm{H}, 0,2.2160036512,-1.2096380673,2.0200452662$

$\mathrm{H}, 0,2.7367331207,1.7238870589,1.3223605694$

$\mathrm{H}, 0,1.7996109793,1.6424671521,-1.3978333392$

C, $0,-2.6527784824,-0.4728849609,-0.3828394499$

$\mathrm{H}, 0,-2.6992441012,-1.3266155725,0.3098677156$

C,0,-1.0735246099,0.5768424052,1.6483486654

$\mathrm{H}, 0,-0.4999769147,0.7722635962,2.5481376176$

$\mathrm{C}, 0,-2.3727444136,0.9283037686,1.6315900435$

$\mathrm{H}, 0,-2.8174999852,1.4095526017,2.5014744565$

C, $0,-3.1991262787,0.7365484439,0.3958390709$

C, $0,-4.6683983039,0.3310267856,0.5781517108$

C,0,-3.7021148501,-0.7044539784,-1.473020528

$\mathrm{H}, 0,-3.5011671283,-0.0670634986,-2.3421027303$

$\mathrm{H}, 0,-3.7137764939,-1.7393339617,-1.8239067341$

C, $0,-5.0390106317,-0.2996824816,-0.7871422939$

$\mathrm{H}, 0,-5.6920695933,-1.1615780478,-0.6189709188$

$\mathrm{H}, 0,-5.5988047858,0.4140815648,-1.3977966895$

C, $0,-3.1701939117,2.0498886042,-0.412684134$

$\mathrm{H}, 0,-3.6025084846,2.8566919034,0.1884684083$

$\mathrm{H}, 0,-2.1455609107,2.3365780524,-0.6647700438$

$\mathrm{H}, 0,-3.7472281562,1.9749044689,-1.340052346$

$0,0,4.8558822442,0.7685948626,-0.1538597176$

$\mathrm{H}, 0,3.4527961823,-0.6952640035,-1.7949097457$

$\mathrm{H}, 0,-5.3034515024,1.1907210457,0.8256575295$ 
$0,0,-4.7458920362,-0.6250083178,1.6401213551$

$\mathrm{H}, 0,-5.649893255,-0.9660407555,1.6712884844$

$0,0,1.3981442337,2.4958731264,-1.1639178199$

$\mathrm{H}, 0,4.452363152,2.6558807631,-0.2808847257$

$\mathrm{O}, 0,4.0110424848,3.5095922941,-0.4405040932$

$\mathrm{H}, 0,3.0926377807,3.2635031333,-0.6531150477$

$\mathrm{H}, 0,0.8530976123,2.2893538698,-0.3896130399$

$\mathrm{O}, 0,1.7486018209,-2.8160259967,0.094124262$

$\mathrm{H}, 0,1.6660387164,-3.3379098398,-0.715535903$

$\mathrm{H}, 0,3.1761809495,-2.8659144819,0.5563761983$

O,0,4.1460634697,-2.9146806588,0.9070194357

$\mathrm{H}, 0,4.5893472827,-2.1504062083,0.5168326178$

trenbolone(SO) - complex with two $\mathrm{H}_{2} \mathrm{O}$ at $\mathrm{C}_{4}$ and $\mathrm{HO}^{-}$and $\mathrm{H}_{2} \mathrm{O}$ at $\mathrm{C}_{5}$

Charge $=-1$ Multiplicity $=1$

C,0,0.874339147,-0.7810998764,-2.0176733985

C, $0,1.6624730156,-0.4312166189,-0.7798243161$

C, $0,0.9549349701,-0.1023166941,0.4611119784$

C, $0,-0.3999064843,0.0745393009,0.4565820695$

C,0,-1.202436192,-0.1106141569,-0.8326817623

C,0,-0.5560606772,-1.1851509496,-1.7017469225

$\mathrm{H}, 0,0.8580871926,0.1146140912,-2.6568908225$

$\mathrm{H}, 0,1.4102261351,-1.5548158554,-2.5766578965$

$\mathrm{H}, 0,-1.1743774969,0.8349215082,-1.3997964029$

$\mathrm{H}, 0,-0.57473113,-2.1439311341,-1.1656484666$

$\mathrm{H}, 0,-1.1236464146,-1.3101562204,-2.6291024686$

C,0,1.8267900093,-0.0293320933,1.6950379058

C, $0,3.0208448699,-0.3914276617,-0.8594612417$

C,0,3.8472798926,0.1676798964,0.1938656509

C, $0,3.1515758442,0.6818481438,1.4246638298$

$\mathrm{H}, 0,1.3262999413,0.4706811687,2.5223649578$

$\mathrm{H}, 0,3.8320056071,0.6031450122,2.276540964$

$\mathrm{H}, 0,2.0339951382,-1.0581675729,2.0208870884$

$\mathrm{H}, 0,2.9762817344,1.7533024873,1.2434176589$

$\mathrm{H}, 0,0.7063083705,2.2408781956,-1.5855522866$

C,0,-2.6492630504,-0.4265804137,-0.4877145347

$\mathrm{H}, 0,-2.6438153495,-1.3655050598,0.0854217793$

C,0,-1.1383077505,0.4343338037,1.6771011219

$\mathrm{H}, 0,-0.5861680637,0.5143074963,2.6066948968$

C, $0,-2.4609978537,0.6889136403,1.6991226858$

$\mathrm{H}, 0,-2.9409777905,0.9849504463,2.6306078977$

C, $0,-3.2681821806,0.6328485688,0.4392126521$

C, $0,-4.7131072095,0.1265265783,0.5469425231$

C, $0,-3.6790842576,-0.5737840373,-1.6108132917$

$\mathrm{H}, 0,-3.5092569675,0.1788046259,-2.3896749271$

$\mathrm{H}, 0,-3.6314644304,-1.5550760863,-2.0890622265$

C, $0,-5.039748887,-0.3333042894,-0.895046135$

$\mathrm{H}, 0,-5.6487405961,-1.2413921625,-0.8534633197$

$\mathrm{H}, 0,-5.6309515402,0.4274477131,-1.4121363989$

C, $0,-3.3042694366,2.0443131722,-0.1815751169$ 
$\mathrm{H}, 0,-3.783265786,2.7380930502,0.5169454107$ $\mathrm{H}, 0,-2.2956115454,2.4151133063,-0.3822948162$ $\mathrm{H}, 0,-3.872586944,2.0626842488,-1.1170801792$ $0,0,5.0703933686,0.3135385759,0.0455450298$ $\mathrm{H}, 0,3.5252388829,-0.6798239403,-1.7792381278$ $\mathrm{H}, 0,-5.3962814087,0.9090152777,0.8995534419$ $0,0,-4.7383923438,-0.9656833927,1.4707937647$ $\mathrm{H}, 0,-5.6244707173,-1.3522679195,1.457166951$ O,0,1.2035908574,2.8299070543,-0.9992492854 $\mathrm{H}, 0,4.4633643155,2.0723667792,-1.3143845754$ $0,0,3.9642746544,2.790066587,-1.7278960618$ $\mathrm{H}, 0,3.0441527103,2.6907502413,-1.417671843$ $\mathrm{H}, 0,1.0840644933,2.4426098635,-0.1193844416$ $0,0,1.764961205,-3.5569025679,0.4697240475$ $\mathrm{H}, 0,1.5665382456,-3.0234169748,-0.3102379252$ $\mathrm{H}, 0,3.048089769,-3.1905866655,0.9281022114$ $\mathrm{O}, 0,4.0395175666,-2.9936704679,1.2779079834$ $\mathrm{H}, 0,3.9823665678,-2.2245350217,1.8578746905$

\section{$17 \beta$}

cis- $12 \mathrm{OH}(\mathrm{SO})$ - complex with $\mathrm{HO}^{-}$and $\mathrm{H}_{2} \mathrm{O}$ at $\mathrm{C}_{3}$ carbonyl and $\mathrm{H}_{2} \mathrm{O}$ and $\mathrm{C}_{12}$

Charge $=-1$ Multiplicity $=1$

C, $0,-2.013022828,-1.68972283,-1.2573429887$

C, $0,-2.4047600724,-0.2525588392,-1.0522836205$

$\mathrm{C}, 0,-1.532534516,0.7354050606,-0.7601884183$

C, $0,-0.1000624965,0.4399863289,-0.5462238647$

C, $0,0.2590149434,-1.0310355027,-0.4078127973$

$\mathrm{C}, 0,-0.509096393,-1.8537932341,-1.4449715996$

$\mathrm{H}, 0,-2.356652153,-2.2699078509,-0.3868799362$

$\mathrm{H}, 0,-2.5593314582,-2.0883489621,-2.1207776288$

$\mathrm{H}, 0,-0.0727880778,-1.3655533414,0.5900323735$

$\mathrm{H}, 0,-0.2207219534,-1.5136684997,-2.4488279952$

$\mathrm{H}, 0,-0.230887194,-2.9098625189,-1.3643594675$

C, $0,-2.0864780755,2.1380276484,-0.6086193905$

C, $0,-3.8734699764,0.0757037439,-1.1816718286$

C, $0,-4.3203288236,1.0459637785,-0.1167706878$

C, $0,-3.3456989129,2.1286969634,0.2639986517$

$\mathrm{H}, 0,-1.3572932185,2.8119259501,-0.1574997272$

$\mathrm{H}, 0,-3.8739659385,3.0870655344,0.2346761194$

$\mathrm{H}, 0,-2.3301619142,2.5480582151,-1.5974045849$

$\mathrm{H}, 0,-3.076831551,1.9422573622,1.3115803409$

$\mathrm{H}, 0,-4.5006514035,-0.818687015,-1.1484317487$

C, $0,1.7632417937,-1.2176021889,-0.5194811078$

$\mathrm{H}, 0,2.0435535182,-0.9880175582,-1.5609400472$

C, $0,0.8398278485,1.3994271124,-0.4620823643$

$\mathrm{H}, 0,0.5696994491,2.4469492397,-0.5877813497$

C, $0,2.3109413855,1.1567020239,-0.2111007816$

$\mathrm{H}, 0,2.8585708541,1.2299867559,-1.1649259023$

C, $0,2.5503406765,-0.2357621385,0.3741766492$ 
C, $0,3.9668698424,-0.8063291714,0.1934221244$

C, $0,2.3279754178,-2.6055342923,-0.1710951078$

$\mathrm{H}, 0,1.7448273995,-3.0587984194,0.6383562559$

$\mathrm{H}, 0,2.2797933266,-3.2853331415,-1.0250412609$

C, $0,3.7825350765,-2.3331206294,0.2847574483$

$\mathrm{H}, 0,4.5237199805,-2.8552479855,-0.3255886712$

$\mathrm{H}, 0,3.9343421063,-2.6419875782,1.3244060307$

C, $0,2.1155811844,-0.3073277615,1.8421296213$

$\mathrm{H}, 0,2.7915000841,0.2729965483,2.4739660028$

$\mathrm{H}, 0,1.1064116039,0.0938820151,1.973634487$

$\mathrm{H}, 0,2.1190116524,-1.3399017088,2.2049773429$

O,0,-5.4189169688,0.9504469829,0.4185201714

$\mathrm{H}, 0,-4.0610038951,0.5764738505,-2.1463338574$

$\mathrm{O}, 0,2.8269702931,2.1636589203,0.6748118856$

$\mathrm{H}, 0,2.4964851679,3.0256887452,0.382838852$

$\mathrm{H}, 0,4.6454000517,2.4044237336,0.1181818473$

$\mathrm{O}, 0,5.5661452424,2.162357697,-0.0867981166$

$\mathrm{H}, 0,5.6844454281,-0.8545488669,1.1339684131$

$\mathrm{H}, 0,4.3193714669,-0.5316875677,-0.8120858836$

$\mathrm{O}, 0,4.8924580245,-0.2986287382,1.1557860589$

$\mathrm{H}, 0,5.6237181518,1.2776077423,0.3147715286$

$0,0,-2.5828179981,-0.7831805349,2.2532768464$

$\mathrm{H}, 0,-5.2577499593,-1.1587542335,1.1540100438$

$0,0,-4.7108499354,-1.8550410309,1.5415006949$

$\mathrm{H}, 0,-3.8005998328,-1.377739214,1.8200809402$

$\mathrm{H}, 0,-2.1408744245,-0.4369135989,1.4664670047$

TS1 cis-12OH(SO) - complex

Charge $=-1$ Multiplicity $=1$

C, $0,-1.8550335444,-1.8243620116,-0.73889104$

C, $0,-2.3037739374,-0.3996864162,-0.5201736127$

C, $0,-1.4284995563,0.6125166948,-0.3008573893$

C, $0,0.0269231127,0.3689012005,-0.2546008749$

C, $0,0.4786531084,-1.0817934813,-0.1976483074$

C, $0,-0.3869201191,-1.9293332728,-1.130279519$

$\mathrm{H}, 0,-2.039257498,-2.3922161863,0.1853242437$

$\mathrm{H}, 0,-2.4925513353,-2.2822389233,-1.5044237565$

$\mathrm{H}, 0,0.3148979421,-1.4491705786,0.8294869656$

$\mathrm{H}, 0,-0.2502373279,-1.5732322876,-2.160586694$

$\mathrm{H}, 0,-0.0599697302,-2.9743970337,-1.0991664073$

C, $0,-1.937034109,2.0315828754,-0.1410767233$

C, $0,-3.778061891,-0.2068153271,-0.5407872479$

C, $0,-4.2848357952,1.1374430219,-0.3623944665$

C, $0,-3.3756096953,2.0824820881,0.38160286$

$\mathrm{H}, 0,-1.3017959798,2.5841750951,0.5571957162$

$\mathrm{H}, 0,-3.7864043115,3.0942248692,0.344013294$

$\mathrm{H}, 0,-1.8698488059,2.562891727,-1.1012544621$

$\mathrm{H}, 0,-3.3870747166,1.7500597619,1.4301079844$

$\mathrm{H}, 0,-4.2243170017,-0.7763935429,0.5332205074$

C, $0,1.9590481703,-1.1867286014,-0.5273900102$ 
$\mathrm{H}, 0,2.0757367842,-0.9177698696,-1.5904002121$ C, $0,0.9271053321,1.3728446791,-0.2696371454$ $\mathrm{H}, 0,0.5922738619,2.4072493822,-0.3393459447$ C, $0,2.4283835587,1.2047353478,-0.2249198696$ $\mathrm{H}, 0,2.8404412197,1.3354741036,-1.239345359$ C,0,2.8191040746,-0.187503956,0.2746751187 C,0,4.220509753,-0.6791025037,-0.1259229221 C,0,2.6336341684,-2.5513849486,-0.3071290945 $\mathrm{H}, 0,2.1987042804,-3.0514784906,0.5656807297$ $\mathrm{H}, 0,2.4917006345,-3.2141666378,-1.1640630044$ C,0,4.1251265616,-2.2156215792,-0.0615024486 $\mathrm{H}, 0,4.7906529367,-2.6807191333,-0.7931554056$ $\mathrm{H}, 0,4.4463372359,-2.5454098599,0.9322067095$ C,0,2.6074024657,-0.3187883568, 1.7865988703 $\mathrm{H}, 0,3.3399811511,0.2786722001,2.3335459508$ $\mathrm{H}, 0,1.6108785961,0.0270668288,2.0753871503$ $\mathrm{H}, 0,2.7129777562,-1.3587196548,2.1113880234$ $\mathrm{O}, 0,-5.4379494506,1.4857654794,-0.6851586878$ $\mathrm{H}, 0,-4.2939688532,-0.7663453544,-1.3274538419$ $\mathrm{O}, 0,3.0100065718,2.2159223155,0.6172742777$ $\mathrm{H}, 0,2.5691187195,3.057924326,0.4325719024$ $\mathrm{H}, 0,4.6976424834,2.5687244457,-0.1863845245$ $0,0,5.5974281627,2.3784867124,-0.5082466296$ $\mathrm{H}, 0,6.0527943739,-0.6732074658,0.5698950813$ $\mathrm{H}, 0,4.4105544546,-0.3591451535,-1.1612654832$ $\mathrm{O}, 0,5.2509852471,-0.1496912032,0.7106782741$ $\mathrm{H}, 0,5.7446379767,1.4870303299,-0.1445891469$ O,0,-4.7499224029,-1.4162006909,1.6227639701 $\mathrm{H}, 0,-6.6752831129,0.0078817461,-0.1249571599$ O,0,-7.0023904152,-0.7287360889,0.4168912607 $\mathrm{H}, 0,-6.1952085785,-1.0110494288,0.9375182419$ $\mathrm{H}, 0,-4.5608095261,-0.8357351918,2.3726382588$

enolate cis- $12 \mathrm{OH}(\mathrm{SO})$ - complex with three $\mathrm{H}_{2} \underline{\mathrm{O}}$ Charge $=-1$ Multiplicity $=1$

C, $0,-1.8390373844,-1.823076755,-0.9252263069$

C, $0,-2.2960207648,-0.3970227394,-0.7169607683$

C, $0,-1.4144838322,0.6085756418,-0.433749946$

C, $0,0.0274602012,0.3634368746,-0.3210799699$

C, $0,0.4726662826,-1.092355262,-0.278623453$

C, $0,-0.3567739884,-1.9206308897,-1.260437097$

$\mathrm{H}, 0,-2.0517675435,-2.3977697767,-0.011360702$

$\mathrm{H}, 0,-2.4455902209,-2.2781720791,-1.7167553162$ $\mathrm{H}, 0,0.2700123428,-1.481733174,0.7334371725$ $\mathrm{H}, 0,-0.1800434363,-1.5448474649,-2.277782608$ $\mathrm{H}, 0,-0.0304384308,-2.9663157765,-1.2368467191$ C,0,-1.9635471513,2.0179767526,-0.3519310298 C, $0,-3.7230154162,-0.1563674697,-0.8400225527$ C, $0,-4.3039514714,1.0264835456,-0.4209682786$ C,0,-3.3838443567,2.0525946888,0.211907881 
$\mathrm{H}, 0,-1.325274546,2.6526544421,0.2689652446$ $\mathrm{H}, 0,-3.8245915036,3.0476569046,0.099549865$ $\mathrm{H}, 0,-1.9632798733,2.4673940739,-1.3585956367$ $\mathrm{H}, 0,-3.3611832774,1.8328110529,1.2917772508$ $\mathrm{H}, 0,-3.955759901,-0.8312090582,1.2038444809$ C, $0,1.9640110551,-1.1985707135,-0.5542948602$ $\mathrm{H}, 0,2.1237430374,-0.8997288555,-1.60370943$ $\mathrm{C}, 0,0.940375627,1.360141208,-0.2683022637$ $\mathrm{H}, 0,0.6184359321,2.3990284682,-0.3292648568$ C,0,2.4361234535,1.1788722572,-0.1622176976 $\mathrm{H}, 0,2.8976635024,1.3395498922,-1.1510547798$ C,0,2.7997576821,-0.2304108698,0.3087900962 C,0,4.2115279472,-0.7197614642,-0.056377027 C, $0,2.6210774915,-2.574217975,-0.349449459$ $\mathrm{H}, 0,2.1520841095,-3.0957077523,0.492763503$ $\mathrm{H}, 0,2.5055117205,-3.2116266623,-1.2293194349$ C,0,4.1050761385,-2.2568398014,-0.0418470152 $\mathrm{H}, 0,4.7930227149,-2.7044802966,-0.7636777853$ $\mathrm{H}, 0,4.3894588189,-2.6185917703,0.9520086541$ C,0,2.5324576528,-0.4063153403,1.8072333205 $\mathrm{H}, 0,3.2517472404,0.1647375458,2.3983846017$ $\mathrm{H}, 0,1.5299457294,-0.0572057249,2.0702875211$ $\mathrm{H}, 0,2.6147323023,-1.4570612236,2.1027374201$ $0,0,-5.5748910363,1.2794622589,-0.4391972652$ $\mathrm{H}, 0,-4.3549515062,-0.9515499288,-1.2320753398$ $\mathrm{O}, 0,2.9890645771,2.1598085635,0.7366540146$ $\mathrm{H}, 0,2.5557250566,3.0080022827,0.5628316792$ $\mathrm{H}, 0,4.7031305599,2.5235126811,0.0238322598$ $0,0,5.6151026482,2.3551203711,-0.27667725$ $\mathrm{H}, 0,6.0220442606,-0.740987789,0.690666805$ $\mathrm{H}, 0,4.4390964799,-0.3705568676,-1.0745944036$ $\mathrm{O}, 0,5.2161079542,-0.2234218716,0.8301613614$ $\mathrm{H}, 0,5.7577687262,1.4484717789,0.0480535665$ O,0,-4.221717278,-1.2199532775,2.0590000095 $\mathrm{H}, 0,-6.4006814787,-0.09444099,0.2005005606$ $\mathrm{O}, 0,-6.7933736884,-0.866954623,0.6771396319$ $\mathrm{H}, 0,-6.0828817632,-1.1511276991,1.2747922512$ $\mathrm{H}, 0,-4.1392373959,-0.4992983428,2.6977921014$

TS2 cis- $12 \mathrm{OH}(\mathrm{SO})$ - complex
Charge $=-1$ Multiplicity $=1$
C,0,-1.7622516304,-2.3220255546,-0.2738772857
C, $0,-2.3314013525,-0.9745339798,-0.6416882169$
C,0,-1.4789980808,0.1550442738,-0.7463926985
C, $0,-0.0990910467,0.0535977335,-0.5201796323$
C, $0,0.4937080163,-1.2755443818,-0.0558046533$
C, $0,-0.2923224197,-2.4470870791,-0.6387984581$
$\mathrm{H}, 0,-1.8764699934,-2.4428509942,0.8139666558$
$\mathrm{H}, 0,-2.3643512188,-3.1080337083,-0.7396977694$
$\mathrm{H}, 0,0.4114943189,-1.3303454596,1.0414924633$ 
$\mathrm{H}, 0,-0.1748048703,-2.4516370551,-1.7310339981$ $\mathrm{H}, 0,0.1124752766,-3.3908751578,-0.2591630883$ C,0,-2.1180115022,1.4411817662,-1.2296837486 C, $0,-3.7086055092,-0.8906414207,-0.8497795182$ C, $0,-4.3845471234,0.3587398598,-0.9302830175$ C, $0,-3.5507855003,1.6008480468,-0.725059964$ $\mathrm{H}, 0,-1.5420040682,2.3111798996,-0.9090796592$ $\mathrm{H}, 0,-4.0503114689,2.450130534,-1.1985988418$ $\mathrm{H}, 0,-2.1231431188,1.453637635,-2.330556592$ $\mathrm{H}, 0,-3.5463800274,1.7889024403,0.3600797962$ $\mathrm{H}, 0,-3.7657631967,-0.6865580506,1.5029860833$ C, $0,1.9606117826,-1.3185239925,-0.4481600088$ $\mathrm{H}, 0,1.9954404918,-1.1995422207,-1.5421837726$ C, $0,0.7918406003,1.1225070746,-0.7915551048$ $\mathrm{H}, 0,0.4067334478,2.0134307625,-1.2767531429$ C, $0,2.1377382378,1.1362322033,-0.4202372223$ $\mathrm{H}, 0,2.802235445,1.8309347402,-0.9276067421$ C, $0,2.7576937045,-0.132338573,0.1399142827$ C, $0,4.1500879176,-0.4912811486,-0.4103437475$ C,0,2.8034589532,-2.5527164816,-0.1130129209 $\mathrm{H}, 0,2.5778274841,-2.9246325537,0.8924175156$ $\mathrm{H}, 0,2.6156275598,-3.3709448423,-0.812636407$ C, $0,4.2588941037,-2.021293501,-0.202338775$ $\mathrm{H}, 0,4.82016488,-2.4848274748,-1.017498216$ $\mathrm{H}, 0,4.8095956546,-2.2098737287,0.7246067245$ C, $0,2.7813748311,-0.1589738004,1.6755397641$ $\mathrm{H}, 0,3.4556686791,0.6103657543,2.0557352367$ $\mathrm{H}, 0,1.7891211606,0.0368744789,2.0877466844$ $\mathrm{H}, 0,3.1261103227,-1.1295773407,2.0436407778$ $\mathrm{O}, 0,-5.6329430554,0.458616139,-1.060785552$ $\mathrm{H}, 0,-4.309513668,-1.7968943388,-0.8615848185$ $\mathrm{O}, 0,2.2023472378,2.5080197248,1.1310063746$ $\mathrm{H}, 0,1.7252265437,3.1847268587,0.6321067583$ $\mathrm{H}, 0,3.6943640866,2.8170141864,1.0424864983$ $\mathrm{O}, 0,4.7072429743,2.9483191654,0.9633497315$ $\mathrm{H}, 0,6.0335558004,-0.0115604614,-0.1733324328$ $\mathrm{H}, 0,4.1715920472,-0.2510244901,-1.4819220301$ $\mathrm{O}, 0,5.1933771722,0.2306983775,0.2398764458$ $\mathrm{H}, 0,5.0202298918,2.0723552149,0.6821960783$ $\mathrm{O}, 0,-3.8068919106,-0.2160059974,2.3517105447$ $\mathrm{H}, 0,-6.164995498,1.1482846852,0.7134867711$ $0,0,-6.1751058849,1.2744072526,1.6773272317$ $\mathrm{H}, 0,-5.3825651094,0.7963177351,1.9843029967$ $\mathrm{H}, 0,-3.0893963684,0.4311142448,2.2995216195$

trans-5OH(SO) - complex with $\mathrm{HO}^{-}$and $\mathrm{H}_{2} \mathrm{O}$ at $\mathrm{C}_{4}$ and $\mathrm{H}_{2} \underline{\mathrm{O} \text { and } \mathrm{C}_{5}}$ Charge $=-1$ Multiplicity $=1$

C, $0,0.8560799823,-1.2236969558,-1.8963496445$

C,0,1.6967796782,-1.0452675324,-0.6330582965

C, $0,0.9548692242,-0.3574238189,0.5068067161$ 
C, $0,-0.3520951057,-0.0160301425,0.4135520263$

C, $0,-1.1962969487,-0.3865064798,-0.8075714544$

C, $0,-0.5869077537,-1.5622570666,-1.5611705328$

$\mathrm{H}, 0,0.875138489,-0.2859428351,-2.4656287127$

$\mathrm{H}, 0,1.3316962983,-1.9906255174,-2.5185416765$

$\mathrm{H}, 0,-1.2077721105,0.4754125763,-1.4938075849$

$\mathrm{H}, 0,-0.6377973786,-2.468340226,-0.9417836374$

$\mathrm{H}, 0,-1.156551213,-1.7549530626,-2.4759186608$

C, $0,1.823224017,-0.1366731379,1.7240910374$

C, $0,2.9762241794,-0.2618021921,-0.990681208$

$\mathrm{C}, 0,3.8301237658,0.0218058537,0.2134848834$

C, $0,3.1191529786,0.6272896899,1.3907365855$

$\mathrm{H}, 0,1.3011657375,0.4004620442,2.5136155401$

$\mathrm{H}, 0,3.7957962594,0.6598990315,2.2477621587$

$\mathrm{H}, 0,2.0916611181,-1.1154738101,2.1396457657$

$\mathrm{H}, 0,2.8615637005,1.6568854101,1.1051886703$

$\mathrm{H}, 0,2.6756462289,0.7061092712,-1.4127138175$

C, $0,-2.6184475405,-0.6524852743,-0.3363187717$

$\mathrm{H}, 0,-2.551504031,-1.4323824921,0.4400828157$

C, $0,-1.0490516083,0.715278372,1.4947327063$

$\mathrm{H}, 0,-0.4583479953,1.0721523546,2.3314144102$

C, $0,-2.3672967332,0.9831688243,1.4927382916$

$\mathrm{H}, 0,-2.8022199402,1.5628353087,2.306337417$

C, $0,-3.2445506132,0.5719722966,0.3483902181$

C, $0,-4.619549836,-0.0157701898,0.6966113763$

C, $0,-3.6925034848,-1.1045326538,-1.3319076453$

$\mathrm{H}, 0,-3.6240422836,-0.5302042017,-2.2628945954$

$\mathrm{H}, 0,-3.5947258687,-2.1616978629,-1.5913350764$

C, $0,-5.0191417159,-0.7968360837,-0.5817425223$

$\mathrm{H}, 0,-5.5680346691,-1.7067079782,-0.3258678174$

$\mathrm{H}, 0,-5.683646733,-0.1709271292,-1.1856863917$

C, $0,-3.4200738806,1.784403442,-0.5871483843$

$\mathrm{H}, 0,-3.8672590825,2.6160005502,-0.034630814$

$\mathrm{H}, 0,-2.4539684833,2.1257513056,-0.9684757017$

$\mathrm{H}, 0,-4.0713338528,1.5581198285,-1.4374593889$

$0,0,5.0311730246,-0.2258445068,0.2282302946$

$\mathrm{H}, 0,3.5543676581,-0.8123477415,-1.7401024164$

$\mathrm{H}, 0,-4.4986019007,-0.709067848,1.5397399502$

O,0,-5.5464093696,0.9996003015,1.067140572

$\mathrm{H}, 0,-6.3625259651,0.5721266632,1.3598679273$

O,0,1.0726443315,2.7640815382,-1.0061066784

$\mathrm{H}, 0,3.9710751921,2.5740498057,-1.4467589254$

$0,0,3.493134932,3.303964407,-1.0344868062$

$\mathrm{H}, 0,2.4619139186,3.0240005479,-1.0172954859$

$\mathrm{H}, 0,0.9084932662,1.9688960661,-0.4816082787$

$0,0,2.0836973545,-2.3391975006,-0.1138741146$

$\mathrm{H}, 0,2.5198217877,-2.8294853715,-0.8285506071$

$\mathrm{H}, 0,3.4331165925,-2.6467241293,1.207318428$

$0,0,4.167446477,-2.8332053433,1.820684215$

$\mathrm{H}, 0,4.7933059055,-2.1101994052,1.6776226417$ 
TS1 trans-5OH(SO) - complex

Charge $=-1$ Multiplicity $=1$

C, $0,0.8307764247,-1.1937746745,-1.8222518657$

C, $0,1.662447796,-1.019534275,-0.5488882903$

C,0,0.8958773606,-0.3650518047,0.5939867885

$\mathrm{C}, 0,-0.4106029787,-0.0272194686,0.4935574114$

C, $0,-1.2388570027,-0.3821931006,-0.7441103379$

C, $0,-0.6139898519,-1.5449753051,-1.5063774782$

$\mathrm{H}, 0,0.8496510475,-0.2548498039,-2.3871874516$

$\mathrm{H}, 0,1.3160452764,-1.953494224,-2.4467810876$

$\mathrm{H}, 0,-1.2485436757,0.4871393674,-1.4210540171$

$\mathrm{H}, 0,-0.6668724543,-2.4584779478,-0.8978924108$

$\mathrm{H}, 0,-1.1747932096,-1.7304390758,-2.4284985789$

C, $0,1.7632253161,-0.1566734462,1.8128268951$

C, $0,2.9175815461,-0.2149555888,-0.8609635194$

C, $0,3.7161981105,0.2828198966,0.227278926$

C, $0,2.9988570273,0.6948894016,1.4943316912$

$\mathrm{H}, 0,1.2191677499,0.295625056,2.6407742116$

$\mathrm{H}, 0,3.714533089,0.6857141901,2.3211321314$

$\mathrm{H}, 0,2.0990297165,-1.140633934,2.1628322738$

$\mathrm{H}, 0,2.6939120304,1.7402286225,1.3360281469$

$\mathrm{H}, 0,2.4635854706,0.9478841212,-1.3066450425$

C, $0,-2.665364849,-0.6643313938,-0.2957710606$

$\mathrm{H}, 0,-2.6029772358,-1.4599546892,0.4651219235$

C,0,-1.1276446557,0.6682098918,1.5855579203

$\mathrm{H}, 0,-0.5497938305,1.0033056382,2.4406143047$

C, $0,-2.4478461462,0.927481072,1.5752005674$

$\mathrm{H}, 0,-2.8984779709,1.4778183657,2.400590575$

C, $0,-3.3075673417,0.5408403214,0.4085048434$

C, $0,-4.6834567238,-0.0627893497,0.7250462368$

C, $0,-3.7243963961,-1.1018421772,-1.3137798084$

$\mathrm{H}, 0,-3.6465292047,-0.5094193543,-2.2327085462$

$\mathrm{H}, 0,-3.6182042542,-2.1533844286,-1.5921320081$

C, $0,-5.0626241757,-0.8153390188,-0.5761447296$

$\mathrm{H}, 0,-5.612981671,-1.7320747833,-0.3494958404$

$\mathrm{H}, 0,-5.7199620397,-0.1770763256,-1.1751377713$

C, $0,-3.4797300581,1.7719615249,-0.5030479853$

$\mathrm{H}, 0,-3.9339664197,2.5904854134,0.0631538996$

$\mathrm{H}, 0,-2.5122542855,2.1233861331,-0.8709808187$

$\mathrm{H}, 0,-4.123921339,1.5615244026,-1.362828543$

$\mathrm{O}, 0,4.9383842814,0.5387168578,0.1150193174$

$\mathrm{H}, 0,3.5105160083,-0.6486833537,-1.6736058458$

$\mathrm{H}, 0,-4.569182559,-0.7749556976,1.553413671$

$\mathrm{O}, 0,-5.6214404365,0.9383157638,1.1072980641$

$\mathrm{H}, 0,-6.4428017484,0.4997757878,1.3672092824$

$\mathrm{O}, 0,1.979130676,2.109521013,-1.736088924$

$\mathrm{H}, 0,4.6402203216,2.5998254979,-0.4481249595$

$0,0,4.1351123643,3.3303322704,-0.8336487466$

$\mathrm{H}, 0,3.2889558753,2.9038001461,-1.1644574704$

$\mathrm{H}, 0,1.2024332408,2.2568645736,-1.1777097744$

$\mathrm{O}, 0,2.0157156813,-2.3491562769,-0.0514923092$ 
$\mathrm{H}, 0,2.4494423781,-2.8204242659,-0.7798236092$ $\mathrm{H}, 0,3.5941160727,-2.3919195094,0.9782750835$ $0,0,4.4414960922,-2.4622783345,1.4590924643$ $\mathrm{H}, 0,5.0298595607,-1.8268577211,1.0293532015$

enolate trans-5OH(SO) - complex with three $\mathrm{H}_{2} \mathrm{O}$ Charge $=-1$ Multiplicity $=1$

C,0,0.844225896,-1.3900976856,-1.7920876438

C, $0,1.6763772133,-1.2262249091,-0.5151205278$

C, $0,0.9283068434,-0.5076988171,0.5988330215$

C, $0,-0.3648971541,-0.1276352892,0.4816190533$

C, $0,-1.1990766024,-0.4840055216,-0.7503632163$

C,0,-0.6145410898,-1.6877918877,-1.4793182456

$\mathrm{H}, 0,0.8963479847,-0.4591794984,-2.3703147094$

$\mathrm{H}, 0,1.3078527353,-2.1716906748,-2.4062463531$

$\mathrm{H}, 0,-1.1612482069,0.3657537332,-1.4510516807$

$\mathrm{H}, 0,-0.701391139,-2.5846786966,-0.8506187075$

$\mathrm{H}, 0,-1.1756557619,-1.8753132492,-2.4010414602$

C, $0,1.8235122508,-0.2311369112,1.7798407887$

C, $0,2.9658616738,-0.5081386649,-0.8192455303$

$\mathrm{C}, 0,3.597670768,0.3548250711,0.032081833$

C,0,2.9701263284,0.7007794594,1.3712382495

$\mathrm{H}, 0,1.2852457329,0.1883986555,2.6295581308$

$\mathrm{H}, 0,3.7621545445,0.6934690819,2.1290106675$

$\mathrm{H}, 0,2.2477130945,-1.1841945339,2.1176856418$

$\mathrm{H}, 0,2.6056940784,1.736855504,1.3071831563$

$\mathrm{H}, 0,1.7397952275,1.4327087155,-1.2453843297$

C, $0,-2.6424713145,-0.6745105808,-0.3105164487$

$\mathrm{H}, 0,-2.6352096958,-1.442015208,0.4810381927$

C, $0,-1.0510473857,0.6480388635,1.5378520399$

$\mathrm{H}, 0,-0.4626044898,0.9809295244,2.3869185403$

$\mathrm{C}, 0,-2.3553963599,0.9773889277,1.5019955797$

$\mathrm{H}, 0,-2.7854683021,1.5816303598,2.3002384746$

C,0,-3.2200243796,0.5950287608,0.3369929037

C, $0,-4.6359186543,0.0921283486,0.652540864$

C,0,-3.7128559491,-1.0864125474,-1.3270214256

$\mathrm{H}, 0,-3.5860706168,-0.5349868674,-2.2658962878$

$\mathrm{H}, 0,-3.6668762123,-2.1522935553,-1.5644114108$

C, $0,-5.0412310087,-0.6911506812,-0.6229133462$

$\mathrm{H}, 0,-5.6477583536,-1.563919249,-0.3676561126$

$\mathrm{H}, 0,-5.6520010258,-0.0407620475,-1.2571767114$

C, $0,-3.3004304213,1.7978946602,-0.6239304268$

$\mathrm{H}, 0,-3.7108417139,2.664600658,-0.0973572804$

$\mathrm{H}, 0,-2.3064874711,2.0749432335,-0.9861890789$

$\mathrm{H}, 0,-3.9424167872,1.5959643978,-1.4875299469$

$0,0,4.7255013207,0.9718877767,-0.2431879517$

$\mathrm{H}, 0,3.4333902356,-0.7340024174,-1.7785667451$

$\mathrm{H}, 0,-4.5810743016,-0.5884084061,1.5127728094$

$0,0,-5.5141323167,1.1666148843,0.9730491346$

$\mathrm{H}, 0,-6.3589184187,0.7925026949,1.2568665236$ 
$0,0,1.2373205324,2.2650023127,-1.3256102898$ $\mathrm{H}, 0,4.2152469487,2.5948880685,-0.5103551908$ $\mathrm{O}, 0,3.8118155123,3.4789445831,-0.7024582856$ $\mathrm{H}, 0,2.8837243739,3.2700218793,-0.9013629442$ $\mathrm{H}, 0,0.5707268534,2.2068142854,-0.6255973427$ $\mathrm{O}, 0,1.9419216002,-2.5733434908,0.0154103005$ $\mathrm{H}, 0,2.3628350758,-3.0798975626,-0.6965167248$ $\mathrm{H}, 0,3.5958463711,-2.5093344037,0.9672406402$ $\mathrm{O}, 0,4.4684858877,-2.551178611,1.4034287592$ $\mathrm{H}, 0,4.9010490493,-1.7264764724,1.1436990504$

TS2 trans-5OH(SO) - complex

Charge $=-1$ Multiplicity $=1$

C, $0,0.9280522204,-1.4060022891,-1.7145880859$

C, $0,1.7303480024,-0.922541302,-0.5273687503$

C, $0,1.0017639717,-0.3353800545,0.6215173059$

C, $0,-0.3335512035,-0.0971083112,0.5515833014$ C, $0,-1.1396765028,-0.506750766,-0.6839306337$ C, $0,-0.5142118674,-1.7259418635,-1.3566279304$ $\mathrm{H}, 0,0.9290836993,-0.5867779261,-2.4483126339$ $\mathrm{H}, 0,1.4463481109,-2.2452253606,-2.1883011488$ $\mathrm{H}, 0,-1.1076480136,0.3215443903,-1.4104927001$ $\mathrm{H}, 0,-0.5658269615,-2.587695105,-0.679364397$ $\mathrm{H}, 0,-1.0750081148,-1.9795174969,-2.2621755433$ C,0,1.9073246629,-0.0168907375,1.7822977867 C,0,3.0296092767,-0.4533127118,-0.7909821495 C,0,3.7085599588,0.4497318614,0.0514490772 C,0,3.0456624295,0.9017061259,1.334588711 $\mathrm{H}, 0,1.3791729069,0.4333830127,2.6219380557$ $\mathrm{H}, 0,3.8176714081,0.9812975048,2.1062801695$ $\mathrm{H}, 0,2.3373888136,-0.9625649258,2.135928269$ $\mathrm{H}, 0,2.6660560134,1.9180512602,1.1544478341$ $\mathrm{H}, 0,1.7118848714,1.5548404178,-1.623884427$ C,0,-2.5868799852,-0.7313875845,-0.2757813685 $\mathrm{H}, 0,-2.58278485,-1.5229690674,0.4912201419$ C, $0,-1.064130374,0.5637612501,1.6489188228$ $\mathrm{H}, 0,-0.5033272798,0.8504823688,2.5321422695$ C, $0,-2.3805410335,0.8421935051,1.6092428162$ $\mathrm{H}, 0,-2.8529013958,1.3621405519,2.4418896689$ C,0,-3.1998356612,0.5062786678,0.3991369118 C, $0,-4.6197928956,-0.019934665,0.6484342039$ C,0,-3.6236481676,-1.1279794893,-1.3334187093 $\mathrm{H}, 0,-3.46956713,-0.5572632332,-2.2565694807$ $\mathrm{H}, 0,-3.5628290317,-2.1884252295,-1.5903830726$ C, $0,-4.9775221298,-0.7556177199,-0.6671230153$ $\mathrm{H}, 0,-5.5952995662,-1.6347555691,-0.4670522491$ $\mathrm{H}, 0,-5.5622675645,-0.0811967313,-1.3008028803$ C,0,-3.2519064099,1.7435625152,-0.5186037302 $\mathrm{H}, 0,-3.7017886411,2.5849507685,0.0164413552$ $\mathrm{H}, 0,-2.2451616857,2.0459565068,-0.8205393657$ 
$\mathrm{H}, 0,-3.8460824804,1.5622806122,-1.4201563255$ $\mathrm{O}, 0,4.8323160589,0.9581181881,-0.2476862536$ $\mathrm{H}, 0,3.5176857888,-0.7496656305,-1.7176170762$ $\mathrm{H}, 0,-4.5847124558,-0.734352108,1.4820542738$ $0,0,-5.517419798,1.0333369958,0.9835951982$ $\mathrm{H}, 0,-6.3699178515,0.6410508079,1.215229572$ $\mathrm{O}, 0,1.2772474619,2.3810123582,-1.3575268535$ $\mathrm{H}, 0,4.3348967365,2.7915230387,-0.5787908884$ $0,0,3.8510469607,3.6017379418,-0.8215300256$ $\mathrm{H}, 0,2.9418394408,3.2917932796,-0.9836054673$ $\mathrm{H}, 0,0.8415213779,2.1494356532,-0.5230587447$ $0,0,1.931490357,-2.7716220504,0.3703016826$ $\mathrm{H}, 0,1.8519787758,-3.372056615,-0.3835363198$ $\mathrm{H}, 0,3.3709961043,-2.7224413699,0.7918860742$ $\mathrm{O}, 0,4.3497502965,-2.7032307772,1.1197119468$ $\mathrm{H}, 0,4.7501433459,-1.9536048926,0.6608127778$

trenbolone(SO) - complex with two $\mathrm{H}_{2} \mathrm{O}$ at $\mathrm{C}_{4}$ and $\mathrm{HO}^{-}$and $\mathrm{H}_{2} \mathrm{O}$ at $\mathrm{C}_{5}$ Charge $=-1$ Multiplicity $=1$

C, $0,0.9604516621,-0.9931718521,-1.8988427944$ C, $0,1.7276457115,-0.4853843903,-0.703264921$ $\mathrm{C}, 0,1.0044353245,-0.0906744635,0.5093486724$ C, $0,-0.3580961796,0.0063155644,0.4991626743$ $\mathrm{C}, 0,-1.1494286762,-0.3237903552,-0.7672236567$ C,0,-0.4502482102,-1.4343494229,-1.5461649873 $\mathrm{H}, 0,0.9044673573,-0.1664207881,-2.6231414783$ $\mathrm{H}, 0,1.5355176974,-1.7911636655,-2.3795572343$ $\mathrm{H}, 0,-1.1678318348,0.5727350108,-1.4093422101$ $\mathrm{H}, 0,-0.425960701,-2.3453606502,-0.9328178958$ $\mathrm{H}, 0,-1.0089131669,-1.6617145032,-2.459463042$ C,0,1.8741772297,0.1420804006,1.7248290856 C,0,3.0800802149,-0.3650367416,-0.7968958616 C, $0,3.8690014156,0.3449499399,0.1922111115$ C, $0,3.144288467,0.9177099961,1.3798035632$ $\mathrm{H}, 0,1.3440228067,0.6740855807,2.5130464788$ $\mathrm{H}, 0,3.8315573828,0.9627460239,2.2285907095$ $\mathrm{H}, 0,2.1559804709,-0.8396035851,2.1310740153$ $\mathrm{H}, 0,2.8934434086,1.9540639192,1.1067447635$ $\mathrm{H}, 0,0.6544805947,2.0471043933,-1.7987997386$ C,0,-2.5776598635,-0.6863274493,-0.3929809249 $\mathrm{H}, 0,-2.519941881,-1.5777773915,0.2521809987$ C,0,-1.114825367,0.4119145778,1.6932709059 $\mathrm{H}, 0,-0.5677184037,0.5826199858,2.6135442818$ C, $0,-2.4491952256,0.5964520107,1.6997960709$ $\mathrm{H}, 0,-2.9471823161,0.9321577908,2.6085712079$ C, $0,-3.2562967925,0.4011138529,0.4532375161$ C, $0,-4.6425403785,-0.2348369237,0.6223998498$ C, $0,-3.5975627036,-0.9876294272,-1.4974388142$ $\mathrm{H}, 0,-3.4832233395,-0.2822352174,-2.3286770635$ $\mathrm{H}, 0,-3.4778445577,-1.9950475238,-1.9032889783$ 
C,0,-4.9656533565,-0.7946326215,-0.7854482955

$\mathrm{H}, 0,-5.5298994209,-1.7278050655,-0.7139734035$

$\mathrm{H}, 0,-5.5927055678,-0.0714211401,-1.3164465683$

C, $0,-3.382595491,1.7554648877,-0.2706840131$

$\mathrm{H}, 0,-3.8722214575,2.4832430878,0.3826903857$

$\mathrm{H}, 0,-2.3963961062,2.1518999295,-0.5279601631$

$\mathrm{H}, 0,-3.9749340816,1.6748923025,-1.1878261033$

$\mathrm{O}, 0,5.0788454585,0.5594913779,0.0199452953$

$\mathrm{H}, 0,3.6010045462,-0.703081445,-1.6901554058$

$\mathrm{H}, 0,-4.562511169,-1.0573271224,1.3458372479$

$0,0,-5.593879193,0.7092060794,1.1017850017$

$\mathrm{H}, 0,-6.4241055208,0.2430965126,1.2688545529$

$\mathrm{O}, 0,1.1063674114,2.6835717556,-1.2253617286$

$\mathrm{H}, 0,4.4174945774,2.1380780101,-1.5149266922$

$\mathrm{O}, 0,3.8465871813,2.7248005524,-2.0298604983$

$\mathrm{H}, 0,2.9462038601,2.6058819453,-1.6723277619$

$\mathrm{H}, 0,0.9998299208,2.3152342811,-0.3354504206$

$\mathrm{O}, 0,1.9234340626,-3.4668259218,0.8108599052$

$\mathrm{H}, 0,1.7256747652,-3.1530102042,-0.0808114766$

$\mathrm{H}, 0,3.2145408844,-3.0297584038,1.1565261102$

$\mathrm{O}, 0,4.2164844413,-2.7790080018,1.4404834917$

$\mathrm{H}, 0,4.1796861088,-1.9134124919,1.865354236$ 
Details of CAS(10,10) Active Space

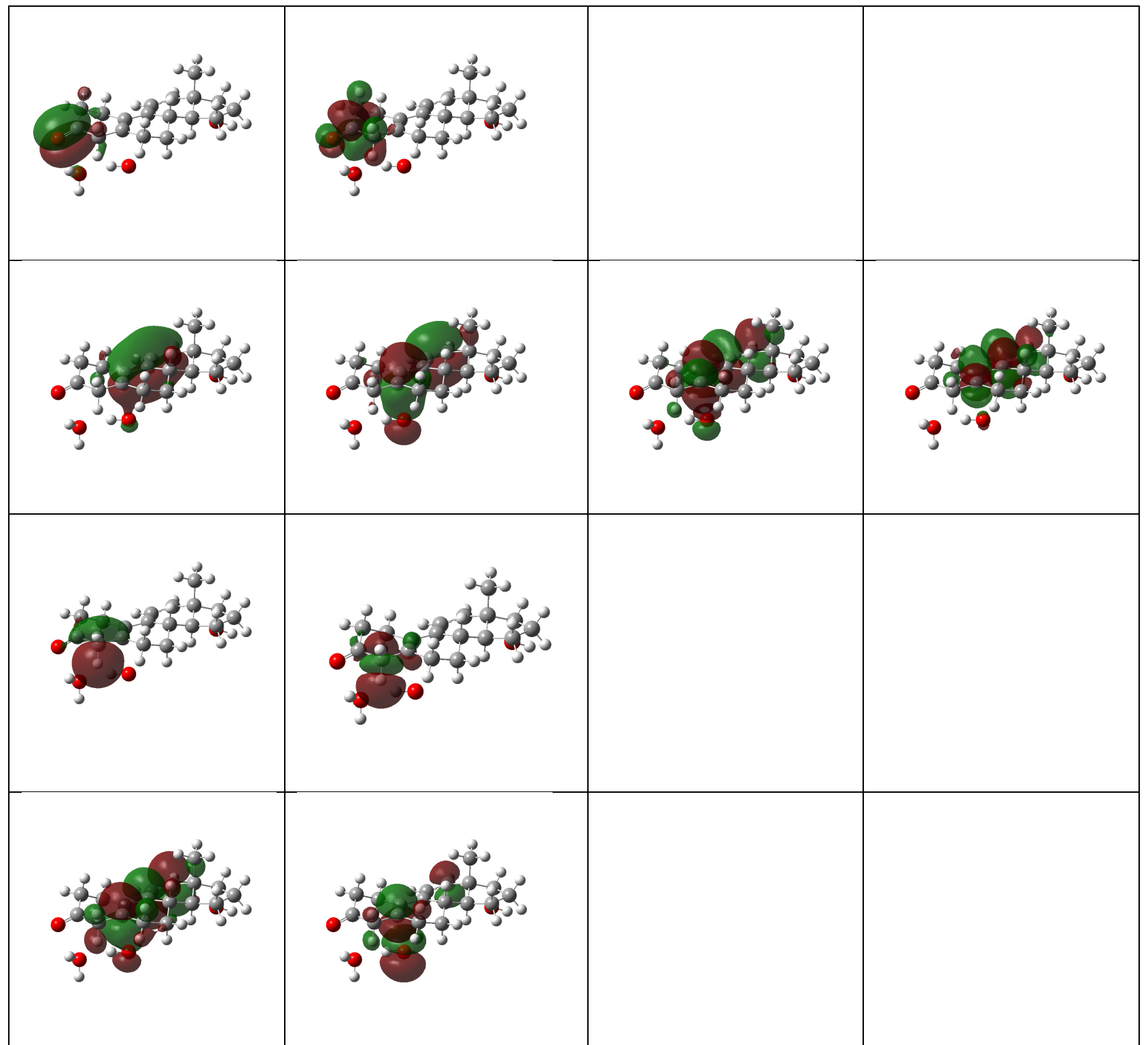

Figure S7. Active space orbitals for first radical intermediate in the photohydration of $17 \alpha-$ trenbolone. From the perspective of the complexed reactants, the active space consists of the 8 $\pi / \pi^{*}$ orbitals of trenbolone plus the one $\sigma / \sigma^{*}$ orbital of water that is transformed into the new C$\mathrm{H}$ and $\mathrm{C}-\mathrm{O}$ bonds in the hydrated products. By the time final products are formed, the active space consists of the two $\pi / \pi^{*}$ orbitals of the $\mathrm{C} 3$ carbonyl (now isolated), the remaining $4 \mathrm{C}=\mathrm{C}$ $\pi / \pi^{*}$ orbitals, the two new $\sigma / \sigma^{*} \mathrm{C}-\mathrm{H}$ orbitals and the two new $\sigma / \sigma^{*} \mathrm{C}-\mathrm{O}$ orbitals. Representative orbitals are shown here for the $17 \alpha$ diradical intermediate. The smaller cc-pVDZ basis was used for these calculations to avoid issues with linear dependence. 


\section{Additional Details for Structures Involved in Photohydration Pathway}

Table S2. Relative energies of photochemically relevant species. Only those species/energies in red are depicted in the main text figures. Energies are relative to $17 \alpha$-trenbolone

\begin{tabular}{|c|c|c|c|}
\hline $\begin{array}{c}\text { Species } \\
\text { (DFT Optimized) }\end{array}$ & $\begin{array}{c}\Delta \mathrm{G}(\mathrm{kcal} / \mathrm{mol}) \\
(\mathrm{DFT})\end{array}$ & $\begin{array}{c}\Delta \mathrm{G}(\mathrm{kcal} / \mathrm{mol}) \\
(\mathrm{CAS})\end{array}$ & $\begin{array}{l}\text { SOMO Occupations } \\
\text { (CAS) }\end{array}$ \\
\hline \multicolumn{4}{|l|}{ alpha } \\
\hline trenbolone (S0) & 0 & 0 & $1.87 / 0.14$ \\
\hline trenbolone $(\mathrm{S} 1 / / \mathrm{S} 0)$ & 83 & 121 & $1.08 / 0.97$ \\
\hline trenbolone $(\mathrm{T} 1 / / \mathrm{S} 0)$ & 55 & 61 & $1.07 / 0.93$ \\
\hline trenbolone (S1) & 76 & - & \\
\hline trenbolone (T1//S1) & 44 & - & \\
\hline trenbolone (S1//T1) & 78 & - & \\
\hline trenbolone (T1) & 38 & 43 & $1.03 / 0.98$ \\
\hline hydration TS (T1) & 77 & 73 & $1.00 / 1.00$ \\
\hline diradical int (T1) & 70 & 59 & $1.00 / 1.00$ \\
\hline diradical int ( $\mathrm{S} 0 \mathrm{sp}$ on $\mathrm{T} 1)$ & - & 59 & $1.10 / 0.90$ \\
\hline $\begin{array}{l}\text { 5OH INT (T1) } \\
\text { (not shown in Figure 3) }\end{array}$ & 71 & 59 & $1.00 / 1.00$ \\
\hline $\begin{array}{l}\text { 5OH INT (S0 sp on T1) } \\
\text { (not shown in Figure 3) }\end{array}$ & - & 59 & $1.11 / 0.89$ \\
\hline $\begin{array}{l}\text { 5OH TS2 (T1) } \\
\text { (not shown in Figure 3) }\end{array}$ & 77 & 78 & $1.02 / 0.98$ \\
\hline $5 \mathrm{OH}$ TS2 (S0 sp on T1) & - & 54 & $1.70 / 0.31$ \\
\hline $\begin{array}{l}\text { 5OH diol (T1) } \\
\text { (not shown in Figure 3) }\end{array}$ & 56 & 58 & $1.02 / 0.98$ \\
\hline $5 \mathrm{OH}$ diol (S0 sp on T1) & - & 27.9 & $1.73 / 0.28$ \\
\hline $5 \mathrm{OH}$ diol ( $\mathrm{S} 0)$ & 6 & 6 & $1.89 / 0.11$ \\
\hline $\begin{array}{l}\text { 12OH INT (T1) } \\
\text { (not shown in Figure 3) }\end{array}$ & 71 & 55 & $1.00 / 1.00$ \\
\hline $\begin{array}{l}\text { 12OH INT (S0 sp on T1) } \\
\text { (not shown in Figure 3) }\end{array}$ & - & 55 & $1.03 / 0.97$ \\
\hline $\begin{array}{l}\text { 12OH TS2 (T1) } \\
\text { (not shown in Figure 3) }\end{array}$ & 85 & 80 & $1.03 / 0.97$ \\
\hline $12 \mathrm{OH}$ TS2 (S0 sp on T1) & - & 52 & $1.71 / 0.30$ \\
\hline $\begin{array}{l}\text { 12OH diol (T1) } \\
\text { (not shown in Figure 3) }\end{array}$ & 55 & 55 & $1.01 / 0.99$ \\
\hline $12 \mathrm{OH}$ diol (S0 sp on T1) & - & 43 & $1.55 / 0.45$ \\
\hline $12 \mathrm{OH}$ diol (S0) & 0 & -1 & $1.90 / 0.10$ \\
\hline
\end{tabular}




\begin{tabular}{|c|c|c|c|}
\hline beta & & & \\
\hline trenbolone $(\mathrm{S} 0)$ & 0 & 1 & $1.87 / 0.14$ \\
\hline trenbolone $(\mathrm{S} 1 / / \mathrm{S} 0)$ & 84 & 122 & $1.08 / 0.98$ \\
\hline trenbolone (T1//S0) & 56 & 62 & $1.07 / 0.93$ \\
\hline trenbolone (T1) & 39 & 43 & $1.03 / 0.98$ \\
\hline hydration TS (T1) & 77 & 70 & $1.00 / 1.00$ \\
\hline diradical int (T1) & 71 & 58 & $1.00 / 1.00$ \\
\hline diradical int ( $\mathrm{S} 0 \mathrm{sp}$ on $\mathrm{T} 1)$ & - & 58 & $1.10 / 0.90$ \\
\hline $\begin{array}{l}\text { 5OH INT (T1) } \\
\text { (not shown in Figure 3) }\end{array}$ & 71 & 58 & $1.00 / 1.00$ \\
\hline $\begin{array}{l}\text { 5OH INT (S0 sp on T1) } \\
\text { (not shown in Figure 3) }\end{array}$ & - & 58 & $1.10 / 0.90$ \\
\hline $\begin{array}{l}\text { 5OH TS2 (T1) } \\
\text { (not shown in Figure 3) }\end{array}$ & 77 & 77 & $1.02 / 0.98$ \\
\hline $5 \mathrm{OH}$ TS2 (S0 sp on T1) & - & 53 & $1.70 / 0.31$ \\
\hline $\begin{array}{l}\text { 5OH diol (T1) } \\
\text { (not shown in Figure 3) }\end{array}$ & 58 & 57 & $1.02 / 0.98$ \\
\hline $5 \mathrm{OH}$ diol (S0 sp on T1) & - & 28 & $1.73 / 0.28$ \\
\hline $5 \mathrm{OH}$ diol (S0) & 6 & 6 & $1.89 / 0.11$ \\
\hline $\begin{array}{l}\text { 12OH INT (T1) } \\
\text { (not shown in Figure 3) }\end{array}$ & 71 & 57 & $1.00 / 1.00$ \\
\hline $\begin{array}{l}\text { 12OH INT (S0 sp on T1) } \\
\text { (not shown in Figure 3) }\end{array}$ & - & 55 & $1.17 / 0.83$ \\
\hline $\begin{array}{l}\text { 12OH TS2 (T1) } \\
\text { (not shown in Figure 3) }\end{array}$ & 84 & 82 & $1.03 / 0.97$ \\
\hline $12 \mathrm{OH}$ TS2 (S0 sp on T1) & - & 49 & $1.74 / 0.27$ \\
\hline $\begin{array}{l}\text { 12OH diol (T1) } \\
\text { (not shown in Figure 3) }\end{array}$ & 56 & 55 & $1.01 / 0.99$ \\
\hline $12 \mathrm{OH}$ diol ( $\mathrm{S} 0 \mathrm{sp}$ on $\mathrm{T} 1)$ & - & 43 & $1.55 / 0.45$ \\
\hline $12 \mathrm{OH}$ diol (S0) & 0 & -2 & $1.90 / 0.10$ \\
\hline
\end{tabular}




\section{Literature Cited}

M.J. Nalbandian, K.E. Greenstein, D. Shuai, M. Zhang, Y.-H. Choa, G.F. Parkin, N.V. Myung, D.M. Cwiertny (2015). Tailored Synthesis of Photoactive $\mathrm{TiO}_{2}$ Nanofibers and $\mathrm{Au} / \mathrm{TiO}_{2}$ Nanofiber Composites: Structure and Reactivity Optimization for Water Treatment Applications. Environmental Science \& Technology, 49, 1654-1663. 